\title{
6D microstate geometries from 10D structures
}

\author{
Stefano Giusto ${ }^{\mathrm{a}, \mathrm{b}, *}$, Luca Martucci $^{\mathrm{a}, \mathrm{b}}$, Michela Petrini ${ }^{\mathrm{c}}$, Rodolfo Russo ${ }^{\mathrm{d}}$ \\ a Dipartimento di Fisica e Astronomia “Galileo Galilei”, Università di Padova, Via Marzolo 8, 35131 Padova, Italy \\ b I.N.F.N. Sezione di Padova, Via Marzolo 8, 35131 Padova, Italy \\ ${ }^{c}$ Laboratoire de Physique Théorique et Hautes Energies, Université Pierre et Marie Curie, CNRS UMR 7589, \\ F-75252 Paris Cedex 05, France \\ ${ }^{\mathrm{d}}$ Centre for Research in String Theory, School of Physics and Astronomy Queen Mary University of London, \\ Mile End Road, London, E1 4NS, United Kingdom
}

Received 24 June 2013; accepted 14 August 2013

Available online 2 September 2013

\begin{abstract}
We use the formalism of Generalised Geometry to characterise in general the supersymmetric backgrounds in type II supergravity that have a null Killing vector. We then specify this analysis to configurations that preserve the same supersymmetries as the D1-D5-P system compactified on a four-manifold. We give a set of equations on the forms defining the supergravity background that are equivalent to the supersymmetry constraints and the equations of motion. This study is motivated by the search of new microstate geometries for the D1-D5-P black hole. As an example, we rewrite the linearised three-charge solution of arXiv:hep-th/ 0311092 in our formalism and show how to extend it to a non-linear, regular and asymptotically flat configuration.
\end{abstract}

(c) 2013 Elsevier B.V. All rights reserved.

\section{Introduction}

Supersymmetric solutions of type II supergravities play a central role in many different areas of string theory, including compactifications with interesting phenomenological properties, the study of holographic gauge/string dualities and the construction of geometries with the same

\footnotetext{
* Corresponding author at: Dipartimento di Fisica ed Astronomia "Galileo Galilei”, Università di Padova, Via Marzolo 8, 35131 Padova, Italy.

E-mail addresses: stefano.giusto@pd.infn.it (S. Giusto), luca.martucci@pd.infn.it (L. Martucci), petrini@1pthe.jussieu.fr (M. Petrini),r.russo@qmul.ac.uk (R. Russo).
} 
charges as black holes. Supersymmetry is also a powerful tool in finding explicit solutions, since it allows to solve first order equations instead of the full set of equations of motion.

In all the examples mentioned above, the supergravity solutions are characterised by the presence of non-trivial Ramond-Ramond (RR) fields, which makes it more complicated to solve the Killing spinor equations. In the past years, several techniques, from G-structures to Generalised Geometry, have been developed to simplify the analysis of the Killing spinor equations in presence of fluxes and make manifest the constraints that supersymmetry puts on the background geometry. In particular, for compactifications to four dimensions, the formalism of Generalised Geometry [1,2] provides a very elegant tool for classifying the internal geometries as well as constructing explicit solutions $[3,4]$ and studying the physics of D-branes $[5,6]$. The idea is that it is possible to give a completely equivalent formulation of the Killing spinor equations as a set of differential equations on two polyforms defined on the internal six-dimensional manifold. The two polyforms are constructed by tensoring the supersymmetry parameters on the six-dimensional space. See [7-11] for previous works reformulating Killing spinor equations in terms of differential forms.

Recently this approach has been generalised in [12] to the analysis of generic ten-dimensional backgrounds in type II supergravity. Again, the supersymmetry constraints are rephrased in terms of forms, built out of the ten-dimensional supersymmetry parameters. In type II supergravities in ten dimensions, supersymmetric backgrounds are characterised by the presence of a Killing vector, which can be either time-like or null. In this paper we will make use of the formalism developed in [12] to study in detail backgrounds with a null Killing vector.

This analysis is motivated by the construction of new microstate geometries for the 5D Strominger-Vafa (SV) black hole $[13,14]$. The SV black hole has a realisation in type IIB string theory compactified on a circle of radius $R \gg \sqrt{\alpha^{\prime}}$ times a string-sized four-dimensional space. For concreteness, in this paper we focus on the $T^{4}$ case, but our results are valid for internal K3 as well. The SV black hole carries three charges which, in string theory language, correspond to D1-branes wrapped on the $S^{1}$, D5's wrapped on $S^{1} \times T^{4}$ and left-moving momentum (in our conventions) along the $S^{1}$. Our main goal is to set up the framework for building horizon-less solutions that carry the same charges as the SV black hole and thus are relevant for the so-called "fuzzball" conjecture (see the review articles [15-21] for a general account of this subject). The first examples of exact and regular supergravity configurations with three charges were found almost ten years ago [22-24] and are part of a class of smooth 1/8-BPS solutions constructed in $[25,26]$.

These geometries are characterised by the presence of a dipole charge for each of the three charges; moreover they are smeared over the compact space $S^{1} \times T^{4}$ (and thus can be studied within a 5D supergravity, see [27] for a recent analysis from this point of view) and are further restricted by the assumption that they admit a tri-holomorphic U(1) isometry.

There is evidence that the generic microstate of the SV black hole does not fall in the class of geometries discussed above. First of all, the assumption of a tri-holomorphic U(1) isometry is clearly too restrictive as it would suppress most of the states already in the two-charge case; this was confirmed by the countings in [28-30]. The assumption of a tri-holomorphic U(1) isometry was relaxed in [31], but a crude counting argument suggested that even those more general solutions could not account for the full three-charge entropy.

Further evidence that the generic microstate geometry might be more general than $[25,26]$ comes from a complementary analysis of D1-D5-P systems based on world-sheet techniques $[32,33]$. The basic idea is that string correlators in flat space capture the large distance behaviour of the gravitational backreaction of each D-brane bound state. This approach has been success- 
fully tested for bound states of D1-D5 branes [34,35] where it was shown that disk correlators with one closed string and all open strings describing the given microstate reproduce the dipole charges for the known two-charge configurations. The same analysis applied to the three-charge geometries [33] suggests that the generic SV microstate is not smeared along the $S^{1}$ and thus should be described within a $6 \mathrm{D}$ supergravity. ${ }^{1}$ Solutions of minimal 6D supergravity coupled to (at most) one tensor multiplet and carrying the same charges and dipole charges as the [25,26] solutions were studied in [38-40]. However, the world-sheet analysis also shows that all fields of type IIB supergravity are non-trivial for general microstates, and not only those necessary for describing the dipoles present in $[25,26]$. This conclusion is supported also by arguments based on the dual D1-D5 CFT [41]. Thus we should expect that for the generic D1-D5-P microstates all type IIB fields are excited and, hence, cannot be described by a restricted ansatz based on minimal six-dimensional supergravity like the one discussed in [38-40].

The first aim of this paper is to provide the most general ansatz and set of equations that should describe the microstates of the D1-D5-P system directly in ten dimensions, with only two simplifying assumptions: we assume that the $T^{4}$ is rigid, i.e. that it appears in the $10 \mathrm{D}$ geometry as a fixed torus apart from an overall scaling function, and that the solution is 'isotropic' on $T^{4}$. Of course, this does not happen for the generic microstate, but, as in the two-charge case, there is the possibility that the class of geometries we consider gives an entropy that has the correct scaling in the large charge limit. Our analysis yields a set of equations generalising the results of [38-40]. The presence of the momentum charge implies the existence of a null Killing vector, while the requirement of supersymmetric D1 and D5 charges is necessary and sufficient to ensure that $1 / 8$ of the total type IIB supersymmetries are preserved.

The second aim of the paper is to provide an explicit solution of our system of equations describing a smooth D1-D5-P microstate geometry that depends non-trivially on the $S^{1}$ coordinate. $^{2}$ A class of such solutions would be provided by the non-linear completion of the perturbative geometries derived from string theory in [33]. However, for sake of simplicity we focus on a different case: we start from the perturbative 6D solution derived in [42] and show that it can be uplifted to $10 \mathrm{D}$ as a two-charge solution of the general type constructed in [41]. Then we can follow the construction of [42], adding a non-trivial momentum and extending the solution to an asymptotically flat geometry. The knowledge of the full 10D equations allows us to perform these steps at the non-linear level and to find a 10D completion of the solution discussed in [42].

The paper is organised as follows. In Section 2 we give the complete set of equations one has to solve in order to have a ten-dimensional supersymmetric solution with a null isometry in type II supergravity. As already mentioned our starting point is the formalism developed in [12]. We construct a one-form, one even/odd polyform in IIA/IIB and a pair of four-forms, which are obtained as (sum of) bilinears in the supersymmetry parameters, and then we give the set of equations on these objects that are equivalent to the Killing spinor equations. In doing that, we derive a new set of equations, which appear simpler than those in [12], but only holds for backgrounds with a null isometry. Moreover, we show that, in order to have a full solution of all

\footnotetext{
1 A first example of solution in this class was found in [36]. The fact that generic microstates should be described by un-smeared six-dimensional geometries was also conjectured in [37] by analysing the supersymmetries preserved by D1-D5-P bound states.

2 In this sense, the geometry we obtain is a regular solution of a non-minimal 6D supergravity; however, the $S^{1}$ is part of the 6D space and the D-branes are wrapped on it: thus from our example it is not possible to derive a regular 'soliton' of $6 \mathrm{D}$ supergravity in the asymptotically Minkowski space $\mathbb{R}^{1,5}$.
} 
supergravity equations of motion, we need also to impose the component of Einstein equations in the null direction. Then in Section 3 we will specify our equations to the study of a D1-D5-P (marginal) bound states. We will consider geometries of the form $\mathbb{R}^{1,1} \times Y \times \hat{Y}$, where $Y$ is fibrated on the null direction in $\mathbb{R}^{1,1}$ and $\hat{Y}$ is eventually identified with the internal $T^{4}$ (or K3). Under the assumption that all fields are homogeneous and isotropic along $\hat{Y}$, we derive the most general ansatz for the type IIB fields describing such systems and the equations that such fields must satisfy in order to give a solution. These first two sections can be skipped by readers who are more interested in the construction of explicit geometries. In order to make the second part of the paper self-contained and consistent with the notation used in the existing literature on microstate geometries, we collect in Appendix E.7 a summary of the equations relevant for the concrete applications to the SV miscrostates. In Section 4, we briefly review the perturbative configuration of [42] and discuss its embedding in the 10D ansatz derived in Section 3. At this point it is easy to write a full regular non-linear solution in the near-horizon region. The extension to an asymptotically flat configuration is trickier: here we present the complete solution but postpone the derivation and a more detailed discussion of its physical properties to a forthcoming paper. Conventions and details of the derivations of Sections 2 and 3 can be found in Appendices A-E.

\section{Null supersymmetric vacua: General discussion}

The study of type II flux backgrounds has shown that, rather than dealing directly with spinorial equations, it is often convenient to rewrite the supersymmetry conditions as differential equations on a set of forms on the compactification manifold. In particular, in [12] this approach has been applied to generic backgrounds in ten dimensions. As we will review in this section, the idea is that, starting from the pair of supersymmetry parameters $\epsilon_{1}$ and $\epsilon_{2}$, one can build a set of forms which can be used to rewrite the ten-dimensional supersymmetry conditions.

Ten-dimensional supersymmetric solutions in type II supergravity are characterised by the existence of a Killing vector $K$, which can be either time-like or null. In this paper we will assume a null $K$. We will re-examine the supersymmetry conditions given in [12] and propose an alternative formulation of some of the supersymmetry constraints.

For generic ten-dimensional backgrounds the supersymmetry constraints are not equivalent to the full set of equations of motion. For the case of $K$ null, we show that, in order to get a proper supergravity solution, the supersymmetry constraints, the Bianchi identities for the NSNS flux and for all the RR fluxes of the democratic formalism, summarised in (2.10), have to be supplemented by the $v v$ component of Einstein equation.

\subsection{Supersymmetry and geometrical structures}

In ten dimensions, a supersymmetric vacuum of type II supergravity is characterised by a pair of Majorana-Weyl spinors, $\epsilon_{1}$ and $\epsilon_{2}$, satisfying the Killing spinor equations. We take their chirality to be

$$
\Gamma_{(10)} \epsilon_{1}=\epsilon_{1}, \quad \Gamma_{(10)} \epsilon_{2}=\mp \epsilon_{2},
$$

where the minus (plus) sign is for IIA (IIB). We have introduced the ten-dimensional chiral operator $\Gamma_{(10)}=\Gamma \underline{0 . .9}$. Here and in the following the uderline denotes flat indices.

Form the spinor bilinears, we can construct a vector $K$ [12]

$$
K=-\frac{1}{2}\left(\bar{\epsilon}_{1} \Gamma^{M} \epsilon_{1}+\bar{\epsilon}_{2} \Gamma^{M} \epsilon_{2}\right) \partial_{M},
$$


which can be either time-like or null. It can be shown $[10,11]$ that $K$ is a Killing vector:

$$
\mathcal{L}_{K} g_{(10)}=0 .
$$

In addition to $K$, one can construct other useful tensorial objects which characterise the geometry. A 1-form

$$
\chi=-\frac{1}{2}\left(\bar{\epsilon}_{1} \Gamma_{M} \epsilon_{1}-\bar{\epsilon}_{2} \Gamma_{M} \epsilon_{2}\right) \mathrm{d} X^{M}
$$

and a polyform

$$
\Psi=-32 \epsilon_{1} \otimes \bar{\epsilon}_{2} \Gamma_{(10)}=\sum_{k} \frac{1}{k !} \bar{\epsilon}_{1} \Gamma_{M_{1} \ldots M_{k}} \epsilon_{2} \mathrm{~d} X^{M_{1}} \wedge \cdots \wedge \mathrm{d} X^{M_{k}},
$$

where $k=$ is even/odd in IIA/IIB. We use conventions which are slightly different from those of [12], since more 'natural' for studying D-branes [43]. ${ }^{3}$

One can then show that the ordinary spinorial supersymmetry conditions, which are obtained by setting to zero the supersymmetry variation of the type II gravitini and dilatini, imply the following differential conditions for $\chi$ and $\Psi$ [12]

$$
\begin{aligned}
& \mathrm{d} \chi=\iota_{K} H, \\
& \mathrm{~d}_{H}\left(e^{-\phi} \Psi\right)=\iota_{K} F+\chi \wedge F,
\end{aligned}
$$

where $\mathrm{d}_{H}$ denotes the twisted exterior derivative

$$
\mathrm{d}_{H} \equiv \mathrm{d}-H \wedge,
$$

$\phi$ is the dilaton, $H$ is the Neveu-Schwarz three-form, and $F$, following the democratic formalism, denotes the sum of all Ramond-Ramond field strengths

$$
F=\sum_{k} F_{k},
$$

with $k$ even (from 0 to 10 ) in IIA and odd (from 1 to 9) in IIB. The redundant degrees of freedom in $F$ are eliminated by the self-duality constraint

$$
F=* \lambda(F),
$$

where $\lambda$ acts on forms by inverting the order of the indices: on a form $F_{k}$ of rank $k$ it acts as $\lambda\left(F_{k}\right)=(-)^{\frac{k(k-1)}{2}} F_{k}$. We assume that the fluxes satisfy the proper the Bianchi identities

$$
\mathrm{d} H=0, \quad \mathrm{~d}_{H} F=0 .
$$

More details on our conventions are given in Appendix A.

The form equations (2.6) and the condition (2.3) can be used to show that $K$ is actually a symmetry of the full solution [12]

$$
\mathcal{L}_{K} H=\mathcal{L}_{K} F=\mathcal{L}_{K} \phi=\mathcal{L}_{K} \Psi=0 .
$$

\footnotetext{
3 The dictionary is the following: $H_{\text {here }}=H_{\text {there }}, F_{\text {here }}^{\text {IIA }}=-F_{\text {there }}^{\mathrm{IIA}}, F_{\text {here }}^{\mathrm{IIB}}=F_{\text {there }}^{\mathrm{IIB}}, K_{\text {here }}=-32 K_{\text {there }}, \chi_{\text {here }}=$ $-32 \tilde{K}_{\text {there }}, \Psi_{\text {here }}^{\mathrm{IIB}}=32 \Phi_{\text {there }}^{\mathrm{IIB}}, \Psi_{\text {here }}^{\mathrm{IIA}}=-32 \Phi_{\text {there }}^{\mathrm{IIA}}$.
} 
As explained in [12], Eqs. (2.3) and (2.6) in general contain less information than the complete set of Killing spinor equations. In other words, they are necessary but not sufficient for having a supersymmetric configuration and must be supplemented by complementary supersymmetry conditions. In [12] a possible way of writing such complementary conditions in terms $\Psi$ and other geometrical data was proposed - see Eqs. (3.1c)-(3.1d) therein. However these extra equations are quite cumbersome to manipulate when looking for explicit solutions. In the following, restricting to the case of $K$ null, we propose another set of complementary conditions which might be easier to work with.

\subsection{The null $K$ case}

Let us assume that $K$ is null. We define a light-cone coordinate $u$ such that

$$
K=\frac{\partial}{\partial u} \text {. }
$$

As explained for instance in [38], one can introduce a second coordinate $v$ and write the (string frame) metric as

$$
\mathrm{d} s_{(10)}^{2}=-2 e^{2 D}(\mathrm{~d} v+\beta)[\mathrm{d} u+\omega+W(\mathrm{~d} v+\beta)]+\mathrm{d} s_{X}^{2},
$$

where $\mathrm{d} s_{X}^{2}=g_{a b}(v, x) \mathrm{d} x^{a} \mathrm{~d} x^{b}$ is a metric on an 8-dimensional space $X, \omega=\omega_{a}(v, x) \mathrm{d} x^{a}$ and $\beta=\beta_{a}(v, x) \mathrm{d} x^{a}$ are one-forms on $X$. In the applications to the D1-D5-P system we will identify

$$
u=\frac{1}{\sqrt{2}}(t-y), \quad v=\frac{1}{\sqrt{2}}(t+y),
$$

where $t$ and $y$ label time and the $S^{1}$. All the fields in the metric can depend in principle on $v$ and the eight-dimensional coordinates $x^{a}$. We choose the following vielbeine and covielbeine

$$
\begin{aligned}
& E^{\underline{u}}=\mathrm{d} u+\omega+W(\mathrm{~d} v+\beta), \quad E_{\underline{u}}=\frac{\partial}{\partial u}, \\
& E^{\underline{v}}=e^{2 D}(\mathrm{~d} v+\beta), \quad E_{\underline{v}}=e^{-2 D}\left(\frac{\partial}{\partial v}-W \frac{\partial}{\partial u}\right), \\
& E^{\underline{a}}=e^{\underline{a}}, \quad E_{\underline{a}}=e_{\underline{a}}^{b}\left(\frac{\partial}{\partial x^{b}}-\omega_{b} \frac{\partial}{\partial u}-\beta_{b} \frac{\partial}{\partial v}\right),
\end{aligned}
$$

such that $\mathrm{d} s_{X}^{2}=\delta_{\underline{a b}} e^{\underline{a}} e^{\underline{b}}$ and $\mathrm{d} s_{(10)}^{2}=-2 E^{\underline{u}} E^{\underline{v}}+\delta_{\underline{a b}} E^{\underline{a}} E^{\underline{b}}$ with, as usual, $e^{\underline{a}} e_{\underline{b}}=\delta_{\underline{b}}^{\underline{a}}$ and $E^{\underline{M}} E_{\underline{N}}=\delta_{\underline{N}}^{\underline{M}}$.

The NSNS and RR field strengths can be split in the following way

$$
\begin{gathered}
H=h+E^{\underline{u}} \wedge h_{\underline{u}}+E^{\underline{v}} \wedge h_{\underline{v}}+E^{\underline{u}} \wedge E^{\underline{v}} \wedge h_{\underline{u v}}, \\
F=f+E^{\underline{u}} \wedge f_{\underline{u}}+E^{\underline{v}} \wedge f_{\underline{v}}+E^{\underline{u}} \wedge E^{\underline{v}} \wedge f_{\underline{u v}},
\end{gathered}
$$

where all the forms $h_{\ldots}$ and $f_{\ldots}$ have components along the 8-dimensional coframe $\mathrm{d} x^{a}$ (or $e^{a}$ ) only. The ten-dimensional self-duality of the RR fields (2.9) translates into the following 8-dimensional relations

$$
\begin{aligned}
& *_{8} \lambda(f)=f_{\underline{u v}}, \quad *_{8} \lambda\left(f_{\underline{u v}}\right)=f, \\
& *_{8} \lambda\left(f_{\underline{u}}\right)=-f_{\underline{u}}, \quad *_{8} \lambda\left(f_{\underline{v}}\right)=f_{\underline{v}} .
\end{aligned}
$$


Notice that this parametrisation, and in particular the choice of $u$ and $v$, is not unique. For fixed $x^{a}$ we have the following mixed diffeomorphisms

$$
u \rightarrow u+U(v, x), \quad v \rightarrow v+V(x), \quad x^{a} \rightarrow x^{a},
$$

which preserve the ansatz (2.13) if

$$
\omega \rightarrow \omega-\mathrm{d}_{X} U+\dot{U} \beta, \quad \beta \rightarrow \beta-\mathrm{d}_{X} V, \quad W \rightarrow W-\dot{U},
$$

with $\dot{U} \equiv \frac{\mathrm{d} U}{\mathrm{~d} v}$. Since we know that $K$ is a symmetry of the background, the forms on $X$ do not depend on $u$ but can in general depend on $v$. On such forms the exterior derivative $\mathrm{d}_{X}=\mathrm{d} x^{a} \wedge \partial_{a}$ is not covariant under (2.18), but can be naturally replaced by the modified exterior derivative:

$$
\mathcal{D} \equiv \mathrm{d}_{X}-\beta \wedge \frac{\mathrm{d}}{\mathrm{d} v} .
$$

$K$ being null also restricts the form of the supersymmetric structures. First of all, we can choose the ten-dimensional gamma matrices to be

$$
\Gamma^{\underline{u}}=-\sqrt{2}\left(\begin{array}{ll}
0 & 0 \\
1 & 0
\end{array}\right) \otimes \gamma_{(8)}, \quad \Gamma^{\underline{v}}=\sqrt{2}\left(\begin{array}{ll}
0 & 1 \\
0 & 0
\end{array}\right) \otimes \gamma_{(8)}, \quad \Gamma^{\underline{a}}=\mathbb{1} \otimes \gamma^{\underline{a}},
$$

where $\gamma^{\underline{a}}$ are eight-dimensional gamma matrices associated with the manifold $X$ and $\gamma_{(8)} \equiv$ $\gamma \frac{1 \ldots 8}{1 s}$ the eight-dimensional chiral operator.

The fact that $K \equiv E_{\underline{u}}$ is null implies that the two supersymmetry parameters $\epsilon_{1}$ and $\epsilon_{2}$ can be written as ${ }^{4}$

$$
\epsilon_{I}=\left(\begin{array}{l}
1 \\
0
\end{array}\right) \otimes \eta_{I} \quad(I=1,2),
$$

where $\eta_{i}$ are real eight-dimensional spinors of positive chirality.

By further imposing the normalisation condition $K=\partial_{u}$ we get

$$
\frac{1}{\sqrt{2}}\left(\eta_{1}^{\dagger} \eta_{1}+\eta_{2}^{\dagger} \eta_{2}\right)=1
$$

so that we can parametrise

$$
\left\|\eta_{1}\right\|^{2} \equiv \eta_{1}^{\dagger} \eta_{1}=\sqrt{2} \sin ^{2} \theta, \quad\left\|\eta_{2}\right\|^{2} \equiv \eta_{2}^{\dagger} \eta_{2}=\sqrt{2} \cos ^{2} \theta
$$

By using this restricted form of the Killing spinors and the gamma matrices given in (2.21), it is not difficult to see that the one-form $\chi$ defined in (2.4) reduces to

$$
\chi=\cos 2 \theta e^{2 D}(\mathrm{~d} v+\beta) .
$$

On the other hand, the polyform $\Psi$ defined in (2.5) becomes

$\overline{4}$ In general one can split the type II supersymmetry parameters as

$$
\epsilon_{I}=\left(\begin{array}{l}
1 \\
0
\end{array}\right) \otimes \eta_{I}+\left(\begin{array}{l}
0 \\
1
\end{array}\right) \otimes \tilde{\eta}_{I}
$$

with $I=1,2$. If $K$ is null, $\bar{\epsilon}_{I} \Gamma^{M} \epsilon_{I} \partial_{M}=\bar{\epsilon}_{I} \Gamma^{\underline{u}} \epsilon_{I} E_{\underline{u}}$ for both $I=1,2$ and then

$$
\epsilon_{I}^{\dagger} \Gamma^{0} \Gamma^{\underline{v}} \epsilon_{I} \sim \epsilon_{I}^{\dagger}\left(-\mathbb{1}+\Gamma^{\underline{01}}\right) \epsilon_{I}=\epsilon_{I}^{\dagger}\left[\left(-\mathbb{1}_{2}+\sigma_{3}\right) \otimes \mathbb{1}\right] \epsilon_{I}=0,
$$

which requires $\tilde{\eta}_{I}=0$. 


$$
\Psi=\sqrt{2} e^{2 D} \sin 2 \theta(\mathrm{d} v+\beta) \wedge \Phi,
$$

where $\Phi$ is a polyform on $X$

$$
\Phi=\sum_{k} \frac{1}{k !} \eta_{1}^{\dagger} \gamma_{a_{1} \ldots a_{k}} \eta_{2} \mathrm{~d} x^{a_{1}} \wedge \cdots \wedge \mathrm{d} x^{a_{k}}
$$

which is odd/even in IIA/IIB.

\subsubsection{The missing supersymmetry conditions}

As shown in [12], Eqs. (2.3) and (2.6) contain less information than the original Killing spinor equations. As we discuss more in detail in Appendix D, the analysis of the intrinsic torsion of the Killing spinor equations and of the system (2.3)-(2.6) reveals that the missing constraints are exactly provided by the vanishing of the $v$-component of the gravitino variations ${ }^{5}$

$$
\begin{aligned}
& \left(\nabla_{\underline{v}}-\frac{1}{4} \iota_{\underline{v}} H\right) \epsilon_{1}+\frac{1}{16} e^{\phi} F \Gamma_{\underline{v}} \Gamma_{(10)} \epsilon_{2}=0, \\
& \left(\nabla_{\underline{v}}+\frac{1}{4} \iota_{\underline{v}} H\right) \epsilon_{2}-\frac{1}{16} e^{\phi} \lambda(F) \Gamma_{\underline{v}} \epsilon_{1}=0,
\end{aligned}
$$

where $F$ is the sum of all RR-field strengths defined in (2.8) and all forms are implicitly contracted by gamma matrices, e.g. $F \equiv \sum_{k} \frac{1}{k !} F_{M_{1} \ldots M_{k}} \Gamma^{M_{1} \ldots M_{k}}$.

In this section, we discuss how one can rewrite the spinorial equations (2.28) as an equivalent set of equations involving just (possibly $v$-dependent) differential forms defined on the internal eight-dimensional space $X$.

Let us introduce a pair of four-forms $\Omega^{(1)}$ and $\Omega^{(2)}$ on $X$ as follows:

$$
\Omega_{a b c d}^{(1)}=\eta_{1}^{T} \gamma_{a b c d} \eta_{1}, \quad \Omega_{a b c d}^{(2)}=\eta_{2}^{T} \gamma_{a b c d} \eta_{2} .
$$

$\Omega^{(1)}$ and $\Omega^{(2)}$ define the two Spin(7) structures associated with the presence of the two Majorana-Weyl spinors $\eta_{1}$ and $\eta_{2}$. Such Spin(7) structures provide a useful tool to analyse the supergravity equations as one can decompose tensors in irreducible representations of these Spin(7) structures. In particular, two-forms on $X$ contain a component transforming as the representations 7 corresponding to $\Omega^{(1)}$ and $\Omega^{(2)}$, which are selected by the following projectors

$$
\begin{aligned}
& \left(P_{7}^{(1)}\right)_{a b}{ }^{c d}=\frac{1}{4}\left(\delta_{[a}^{[c} \delta_{b]}^{d]}-\frac{1}{2 \sqrt{2} \sin ^{2} \theta} \Omega_{a b}^{(1) c d}\right), \\
& \left(P_{7}^{(2)}\right)_{a b}{ }^{c d}=\frac{1}{4}\left(\delta_{[a}^{[c} \delta_{b]}^{d]}-\frac{1}{2 \sqrt{2} \cos ^{2} \theta} \Omega_{a b}^{(2) c d}\right) .
\end{aligned}
$$

Then, as shown in details in Appendix D, the two spinorial equations (2.28) can be recast into the following equivalent set of equations

$$
\begin{aligned}
& \frac{\mathrm{d}}{\mathrm{d} v}(\cos 2 \theta)=\frac{\sqrt{2}}{4} e^{2 D+\phi} f_{\underline{v}} \cdot \Phi, \\
& \frac{1}{\sqrt{2}} e^{\phi}\left(f \cdot \iota_{a} \Phi\right) \mathrm{d} x^{a}=e^{-2 D} \mathcal{D} e^{2 D}-\dot{\beta}+\cos 2 \theta h_{\underline{u v}},
\end{aligned}
$$

\footnotetext{
5 See Appendix A for our convention on the Killing spinor equations.
} 


$$
\begin{aligned}
\frac{1}{\sqrt{2}} e^{\phi}\left(\iota_{a} f \cdot \Phi\right) \mathrm{d} x^{a}=- & h_{\underline{u v}}-\cos 2 \theta\left(e^{-2 D} \mathcal{D} e^{2 D}-\dot{\beta}\right), \\
\iota_{[a} \Omega^{(1)} \cdot \frac{\mathrm{d}}{\mathrm{d} v}\left(\iota_{b]} \Omega^{(1)}\right)= & -16 \sin ^{4} \theta e^{2 D}\left(P_{7}^{(1)}\right)_{a b}{ }^{c d}\left(\mathcal{D} \omega+W \mathcal{D} \beta-h_{\underline{v}}\right)_{c d} \\
& +\sqrt{2} \sin ^{2} \theta e^{2 D+\phi} \Phi \cdot\left(\gamma_{a b} f_{\underline{v}}\right), \\
\iota_{[a} \Omega^{(2)} \cdot \frac{\mathrm{d}}{\mathrm{d} v}\left(\iota_{b} \Omega^{(2)}\right)= & -16 \cos ^{4} \theta e^{2 D}\left(P_{7}^{(2)}\right)_{a b}{ }^{c d}\left(\mathcal{D} \omega+W \mathcal{D} \beta+h_{\underline{v}}\right)_{c d} \\
& +\sqrt{2} \cos ^{2} \theta e^{2 D+\phi} \Phi \cdot\left(f_{\underline{v}} \gamma_{a b}\right) .
\end{aligned}
$$

In the above equations, the gamma matrices $\gamma_{a}$ must be considered as the corresponding operators under Clifford map: if $\omega_{p}$ is a $p$-form, then

$$
\begin{aligned}
& \gamma_{a} \omega_{p} \equiv \iota_{a} \omega_{p}+g_{a b} \mathrm{~d} x^{b} \wedge \omega_{p}, \\
& \omega_{p} \gamma_{a} \equiv(-)^{p-1}\left(\iota_{a} \omega_{p}-g_{a b} \mathrm{~d} x^{b} \wedge \omega_{p}\right) .
\end{aligned}
$$

Furthermore, as usual, $\left.\gamma_{a b} \equiv \gamma_{[a} \gamma_{b}\right]$. Notice that in (2.31d) and (2.31d) only the 7 components (with respect to $\Omega^{(1)}$ and $\Omega^{(2)}$ respectively) of the antisymmetric two-tensor are non-trivial.

Eqs. (2.31a)-(2.31e) provide, for the case of $K$ null, a set of constraints, alternative to Eqs. (3.1c)-(3.1d) of [12], that need to be imposed in addition to (2.3) and (2.6) in order to have a supersymmetric configuration.

\subsubsection{Einstein and B-field equations}

It is well known that supersymmetry does not generically imply the complete set of equations of motion. The question is then which is the minimal set of equations one has to solve in order to be sure to have a solution of the full system of equations of motion. The general strategy is to first impose the supersymmetry and Bianchi identities for the fluxes, and then to check whether these imply the flux and dilaton equations of motion and Einstein equations.

In ten dimensions, one has again to distinguish between backgrounds with null or time-like Killing vectors. We show in Appendix $C$ that for $K$ null, one has to solve the following set of equations: the supersymmetry constraints, the Bianchi identities (2.10) and the $\underline{v v}$ component of the Einstein equations

$$
\mathcal{R}_{\underline{v} \underline{v}}+2 \nabla_{\underline{v}} \nabla_{\underline{v}} \phi-\frac{1}{2} \iota_{\underline{v}} H \cdot \iota_{\underline{v}} H-\frac{1}{4} e^{2 \phi} \iota_{\underline{v}} F \cdot \iota_{\underline{v}} F=0
$$

where $\iota_{\underline{v}} F \cdot \iota_{\underline{v}} F=\sum_{k} \iota_{\underline{v}} F^{(2 k+1)} \cdot \iota_{\underline{v}} F^{(2 k+1)}$ with $k=1 / 2, \ldots, 3 / 2$ for IIA and $k=0, \ldots, 4$ for IIB.

All other equations, namely the equation of motion for $B$ and the dilaton, and the other component of Einstein equations, are automatically satisfied.

With our ansatz for the metric and the fluxes the $\underline{v v}$ component of Einstein equations becomes

$$
\begin{aligned}
0= & -e^{-4 D} \mathcal{D}_{a}\left(e^{2 D} L^{a}\right)+\frac{1}{2} e^{-2 D} \beta_{a} L^{a} g^{b c} \dot{g}_{b c}+2 e^{-2 D} \dot{\beta}_{a} L^{a} \\
& +\frac{1}{4}(\mathcal{D} \omega+W \mathcal{D} \beta)_{a b}(\mathcal{D} \omega+W \mathcal{D} \beta)^{a b}-\frac{1}{4} \frac{\mathrm{d}}{\mathrm{d} v}\left(e^{-4 D} g^{a b}\right) \dot{g}_{a b} \\
& -\frac{1}{2} e^{-4 D} g^{a b} \ddot{g}_{a b}+e^{-2 D} \frac{\mathrm{d}}{\mathrm{d} v}\left(e^{-2 D} \dot{\phi}\right)+e^{-2 D} L^{a} \mathcal{D}_{a} \phi \\
& -\frac{1}{2} h_{\underline{v}} \cdot h_{\underline{v}}-\frac{1}{4} e^{2 \phi} f_{\underline{v}} \cdot f_{\underline{v}},
\end{aligned}
$$


where $h_{\underline{v}}$ and $f_{\underline{v}}$ are defined in (2.16) and the symbol $L$ denotes

$$
L=\dot{\omega}+W \dot{\beta}-\mathcal{D} W
$$

\subsubsection{Summary of BPS equations for null Killing vectors}

In the previous sections we derived a set of conditions on the geometric structures $\chi, \Psi$, $\Omega^{(1)}$ and $\Omega^{(2)}$ which, for null $K$, are equivalent to the standard Killing spinor equations of ten-dimensional type II supergravities. We will summarise them in this section.

First, in addition to $\mathcal{L}_{K} g_{(10)}=0$, we have Eqs. (2.6) [12]

$$
\begin{aligned}
& \mathrm{d} \chi=\iota_{K} H, \\
& \mathrm{~d}_{H}\left(e^{-\phi} \Psi\right)=\iota_{K} F+\chi \wedge F,
\end{aligned}
$$

which must be supplemented by the additional constraints ${ }^{6}$

$$
\begin{aligned}
& e^{-2 D} \mathcal{D} e^{2 D}-\dot{\beta}+\mathcal{D} \log (\sin 2 \theta)=\frac{1}{\sqrt{2} \sin ^{2}(2 \theta)} e^{\phi}\left(f \cdot \iota_{a} \Phi\right) \mathrm{d} y^{a}, \\
& \mathcal{D}_{a} \cos 2 \theta=\frac{1}{\sqrt{2}} e^{\phi} \iota_{a} f \cdot \Phi, \\
& \frac{\mathrm{d}}{\mathrm{d} v}(\cos 2 \theta)=\frac{\sqrt{2}}{4} e^{2 D+\phi} f_{\underline{v}} \cdot \Phi, \\
& \left.\iota_{[a} \Omega^{(1)} \cdot \frac{\mathrm{d}}{\mathrm{d} v}\left(\iota_{b}\right] \Omega^{(1)}\right)=-16 \sin ^{4} \theta e^{2 D}\left(P_{7}^{(1)}\right)_{a b}{ }^{c d}\left(\mathcal{D} \omega+W \mathcal{D} \beta-h_{\underline{v}}\right)_{c d} \\
& +\sqrt{2} \sin ^{2} \theta e^{2 D+\phi} \Phi \cdot\left(\gamma_{a b} f_{\underline{v}}\right), \\
& \left.\iota_{[a} \Omega^{(2)} \cdot \frac{\mathrm{d}}{\mathrm{d} v}\left(\iota_{b}\right] \Omega^{(2)}\right)=-16 \cos ^{4} \theta e^{2 D}\left(P_{7}^{(2)}\right)_{a b}{ }^{c d}\left(\mathcal{D} \omega+W \mathcal{D} \beta+h_{\underline{v}}\right)_{c d} \\
& +\sqrt{2} \cos ^{2} \theta e^{2 D+\phi} \Phi \cdot\left(f_{\underline{v}} \gamma_{a b}\right) \text {. }
\end{aligned}
$$

Second, in order to have a solution of the full set of equations of motion, we also have to impose the Bianchi identities/eom $\mathrm{d} H=0$ and $\mathrm{d}_{H} F=0$ and the $\underline{v v}$ component of Einstein equations

$$
\begin{aligned}
0= & -e^{-4 D} \mathcal{D}_{a}\left(e^{2 D} L^{a}\right)+\frac{1}{2} e^{-2 D} \beta_{a} L^{a} g^{b c} \dot{g}_{b c}+2 e^{-2 D} \dot{\beta}_{a} L^{a} \\
& +\frac{1}{4}(\mathcal{D} \omega+W \mathcal{D} \beta)_{a b}(\mathcal{D} \omega+W \mathcal{D} \beta)^{a b}-\frac{1}{4} \frac{\mathrm{d}}{\mathrm{d} v}\left(e^{-4 D} g^{a b}\right) \dot{g}_{a b} \\
& -\frac{1}{2} e^{-4 D} g^{a b} \ddot{g}_{a b}+e^{-2 D} \frac{\mathrm{d}}{\mathrm{d} v}\left(e^{-2 D} \dot{\phi}\right)+e^{-2 D} L^{a} \mathcal{D}_{a} \phi \\
& -\frac{1}{2} h_{\underline{v}} \cdot h_{\underline{v}}-\frac{1}{4} e^{2 \phi} f_{\underline{v}} \cdot f_{\underline{v}},
\end{aligned}
$$

where $h_{\underline{v}}, f_{\underline{v}}$ and $L$ are defined in (2.16) and (2.35).

\footnotetext{
6 These equations are a partial rewriting of the constraints discussed in Section 2.2.1. In particular, (2.37a) and (2.37b) can be obtained from (2.31a) and (2.31b) by using (2.36a).
} 


\section{The D1-D5-P geometries}

In this section we will apply the formalism developed in Section 2 to study the full backreaction of a D1-D5-P system, where the D1-branes wrap an $S^{1} \subset \mathbb{R}^{1,1}$ while the D5's wrap $S^{1} \times T^{4}$. The momentum represents a left-moving wave propagating on $S^{1}$. We will therefore consider spaces of the form $\mathbb{R}^{1,1} \times Y \times \hat{Y}$, with metric

$$
\mathrm{d} s_{(10)}^{2}=-2 e^{2 D}(\mathrm{~d} v+\beta)[\mathrm{d} u+\omega+W(\mathrm{~d} v+\beta)]+e^{2 G} \mathrm{~d} s_{4}^{2}+e^{2 \hat{G}} \mathrm{~d} \hat{s}_{4}^{2} .
$$

Eventually we will take $\hat{Y}=T^{4}$ with a flat metric, but the following arguments work also when $\hat{Y}$ has Ricci-flat hyper-Kähler metric $\mathrm{d} s_{\mathrm{K} 3}^{2}$.

On the other hand, being $\mathrm{d} s_{4}^{2}$ a priory completely arbitrary, the warping $e^{2 G}$ is a 'pure-gauge' degree of freedom, in the sense that we have the freedom to perform the gauge transformation

$$
G \rightarrow \Lambda \text { and } \mathrm{d} s_{4}^{2} \rightarrow e^{-2 \Lambda} \mathrm{d} s_{4}^{2},
$$

with $\Lambda$ an arbitrary function. We will fix this redundancy by making a convenient gauge choice suggested by the equations.

At different steps in our derivation, we will also make the simplifying assumption that the backgrounds are 'isotropic' along $\hat{Y}$. Configurations describing more general states of the D1-D5-P system can in principle be included by generalising our results.

\subsection{Restricted spinorial structure}

We now derive the restrictions that a D1-D5-P (marginal) bound state puts on the form of the Killing spinors. In this section we will assume that the NSNS two-form has no legs on $\hat{Y}$ and

$$
\mathcal{L}_{K} B=0 .
$$

According to the structure of the metric (3.1), we take a factorised form for the eight-dimensional gamma-matrices $^{7}$

$$
\gamma_{(8)}^{i}=\gamma^{i} \otimes \mathbb{1}, \quad \gamma_{(8)}^{\frac{4+a}{8}}=\gamma_{(4)} \otimes \hat{\gamma}^{a},
$$

and the eight-dimensional spinors $\eta_{I}$

$$
\eta_{I}=\zeta_{I}^{+} \otimes \hat{\zeta}_{I}^{+}+\zeta_{I}^{+\mathrm{c}} \otimes \hat{\zeta}_{I}^{+\mathrm{c}}+\zeta_{I}^{-} \otimes \hat{\zeta}_{I}^{-}+\zeta_{I}^{-\mathrm{c}} \otimes \hat{\zeta}_{I}^{-\mathrm{c}}
$$

with $\zeta_{\alpha}^{ \pm}$and $\hat{\zeta}_{\alpha}^{ \pm}$chiral spinors on the four non-compact spatial directions $Y$ and $\hat{Y}$. The suffix ${ }^{\mathrm{c}}$ denotes the conjugation $\zeta^{+\mathrm{c}}=C_{(4)} \zeta^{+*}$ (see Appendix A for notations and conventions).

Let us first consider what are the constraints a momentum in the direction of $S^{1}$ sets on the spinors $\epsilon_{I}$. A wave propagating left-wise in the direction $y$ is supersymmetric if

$$
\Gamma \underline{u v} \epsilon_{I}=\epsilon_{I} .
$$

This corresponds to the existence of the null Killing vector $K$ and is automatically satisfied by the spinor ansatz (2.22)

$$
\epsilon_{I}=\left(\begin{array}{l}
1 \\
0
\end{array}\right) \otimes \eta_{I} \quad(I=1,2) .
$$

\footnotetext{
7 From now on we use $\gamma^{i}$ and $\hat{\gamma}^{a}$ for the 4D gamma matrices in $Y$ and $\hat{Y}$ respectively and introduce a subscript when we refer to the $8 \mathrm{D}$ gamma matrices.
} 
We then require that a D1-brane probe, filling the $(u, v)$ directions and sitting at a generic point of the internal eight-dimensional space, is always supersymmetric. The supersymmetry condition for a probe D1-brane is

$$
\Gamma_{\mathrm{D} 1} \epsilon_{2}=-\epsilon_{1} .
$$

In general,

$$
\Gamma_{\mathrm{D} 1}=\frac{\epsilon^{\alpha \beta}}{2 \sqrt{-\operatorname{det} \iota^{*}(g-B)}}\left(\Gamma_{\alpha \beta}-B_{\alpha \beta}\right),
$$

where $\sigma^{\alpha}$, with $\alpha=0,1$, denote world-volume coordinates, $\Gamma_{\alpha}=\partial_{\alpha} X^{M} E_{M}^{M} \Gamma_{\underline{M}}$ and $\iota^{*} g \equiv$ $g_{\alpha \beta} \mathrm{d} \sigma^{\alpha} \mathrm{d} \sigma^{\beta}$ and $\iota^{*} B \equiv \frac{1}{2} B_{\alpha \beta} \mathrm{d} \sigma^{\alpha} \wedge \mathrm{d} \sigma^{\beta}$ are the pull-back of the metric and NSNS two-form on the world-volume. Notice that, since we consider D1-brane probes with no induced charges, the world-volume gauge field is zero.

We can use the supersymmetry condition (2.36a) and the fact that $K$ is a symmetry of the solution to determine the form of the NSNS two-form. Indeed (2.36a) is solved by taking

$$
\iota_{K} B=-\chi=-e^{2 D} \cos 2 \theta(\mathrm{d} v+\beta),
$$

which means

$$
B=-e^{2 D} \cos 2 \theta(\mathrm{d} u+\omega) \wedge(\mathrm{d} v+\beta)+b \wedge(\mathrm{d} v+\beta)+\mathcal{B},
$$

where $b$ and $\mathcal{B}$ are a 1 - and 2-form on the internal eight-dimensional space $X$.

By using the metric (2.13) and (3.11) we find that, in our case,

$$
\Gamma_{\mathrm{D} 1}=\frac{1}{\sin 2 \theta}\left(\Gamma_{\underline{u v}}+\cos 2 \theta \mathbb{1}_{(10)}\right)=\frac{1}{\sin 2 \theta}\left(-\sigma_{3}+\cos 2 \theta \mathbb{1}_{(2)}\right) \otimes \mathbb{1}_{(8)},
$$

where in the last step we have used the $2+8$ decomposition (2.21) of the gamma matrices. Hence, the projection (3.8) on the spinors (3.7) reduces to

$$
\frac{\sin \theta}{\cos \theta} \eta_{2}=\eta_{1}
$$

which implies that the two internal eight-dimensional spinors $\eta_{1,2}$ are proportional. Comparison with the normalisation condition (2.24) fixes ${ }^{8}$

$$
\eta_{1}=2^{\frac{1}{4}} \sin \theta \eta, \quad \eta_{2}=2^{\frac{1}{4}} \cos \theta \eta,
$$

where the Majorana-Weyl spinor $\eta$ has positive chirality and unitary norm.

Finally we have to impose that a D5-brane probe wrapping $\hat{Y}$, extending along the $(u, v)$ direction and sitting at any point of $\hat{Y}$ is supersymmetric

$$
\Gamma_{\mathrm{D} 5} \epsilon_{2}=\epsilon_{1} .
$$

As for the D1-brane, there is no purely world-volume gauge field. Since we are assuming vanishing $B$-field along $\hat{Y}$, we can rewrite the D5 projector in terms of the D1 one

$$
\Gamma_{\mathrm{D} 5}=\Gamma_{\mathrm{D} 1}\left(\mathbb{1} \otimes \mathbb{1} \otimes \hat{\gamma}_{(4)}\right) .
$$

We see that (3.15) reduces the spinor (3.5) to

\footnotetext{
8 Notice also that Majorana conditions $\eta_{\alpha}^{\dagger}=\eta_{\alpha}^{T} C_{(8)}$ imply that the proportionality constants must be real.
} 


$$
\eta=\zeta \otimes \hat{\zeta}+\zeta^{\mathrm{c}} \otimes \hat{\zeta}^{\mathrm{c}}
$$

where $\zeta$ and $\hat{\zeta}$ have positive chirality in four dimensions $\left(\gamma_{(4)} \zeta=\zeta, \hat{\gamma}_{(4)} \hat{\zeta}=\hat{\zeta}\right)$ and we can choose them to have unitary norm.

With the ansatz (3.14)-(3.17) for the eight-dimensional spinors, the polyform $\Psi$ takes the form (2.26) with

$$
\Phi=\frac{1}{\sqrt{2}}\left(1+\Omega+e^{4 G+4 \hat{G}} \operatorname{vol}_{4} \wedge \widehat{\operatorname{vol}}_{4}\right),
$$

where $\Omega$ is the four-form defining the $\operatorname{Spin}(7)$ structure associated with $\eta$ (see Appendix B for some definitions and properties of Spin(7) structures)

$$
\Omega=e^{4 G} \mathrm{vol}_{4}+e^{4 \hat{G}} \widehat{\mathrm{vol}}_{4}-e^{2 G+2 \hat{G}} \sum_{A=1}^{3} J_{A} \wedge \hat{J}_{A} .
$$

Furthermore, the forms (2.29) can be expressed in terms of $\Omega$ too

$$
\Omega^{(1)}=\sqrt{2} \sin ^{2} \theta \Omega, \quad \Omega^{(2)}=\sqrt{2} \cos ^{2} \theta \Omega .
$$

In the equation above $\operatorname{vol}_{4}$ and $\widehat{\mathrm{vol}}_{4}$ denote the volume forms on $Y$ and $\hat{Y}$ respectively, while $J_{A}$ and $\hat{J}_{A}(A=1,2,3)$ are two triplets of two-forms which define two $\mathrm{SU}(2)$ structures on $Y$ and $\hat{Y}$, respectively. They are built as bilinears of the spinors $\zeta$ and $\hat{\zeta}$ as follows:

$$
\begin{aligned}
& J_{1}+\mathrm{i} J_{2}=\frac{1}{2} \zeta^{T} C_{(4)} \gamma_{i j} \zeta \mathrm{d} x^{i} \wedge \mathrm{d} x^{j}, \quad J_{3}=\frac{\mathrm{i}}{2} \zeta^{\dagger} \gamma_{i j} \zeta \mathrm{d} x^{i} \wedge \mathrm{d} x^{j}, \\
& -\hat{J}_{1}+\mathrm{i} \hat{J}_{2}=\frac{1}{2} \hat{\zeta}^{T} \hat{C}_{(4)} \hat{\gamma}_{a b} \hat{\zeta} \mathrm{d} \hat{x}^{a} \wedge \mathrm{d} \hat{x}^{a}, \quad \hat{J}_{3}=\frac{\mathrm{i}}{2} \hat{\zeta}^{\dagger} \hat{\gamma}_{a b} \hat{\zeta} \mathrm{d} \hat{x}^{a} \wedge \mathrm{d} \hat{x}^{a} .
\end{aligned}
$$

Both triplets of two-forms are anti-self-dual with respect to the corresponding metrics

$$
*_{4} J_{A}=-J_{A} \quad \text { and } \quad \hat{*}_{4} \hat{J}_{A}=-\hat{J}_{A} \quad A=1,2,3,
$$

and satisfy the usual properties of almost hyper-Kähler structures, e.g.

$$
\left(J_{A}\right)^{i}{ }_{k}\left(J_{B}\right)^{k}{ }_{j}=\sum_{B} \epsilon_{A B C}\left(J_{C}\right)^{i}{ }_{j}-\delta_{A B} \delta_{j}^{i},
$$

or equivalently

$$
J_{A} \wedge J_{B}=-2 \delta_{A B} \text { vol }_{4} .
$$

The same equations hold for $\hat{J}_{A}$.

\subsection{Minimal set of equations for our ansatz}

The second part of the paper will be devoted to the construction of new examples of D1-D5-P geometries describing microstates of a black hole. In this section we specify the general supersymmetry conditions of Section 2.2.3 to the restricted spinorial structure we worked out in Section 3.1 and we identify a minimal set of equations which need to be solved in order to get a full supergravity solution. 
For concreteness, in the rest of the paper we will always take $\hat{Y}=T^{4}$, choosing $\mathrm{d} \hat{s}_{4}^{2}$ to be a flat metric on it. ${ }^{9}$ Then we can take the triplet $\hat{J}_{A}$ to be closed

$$
\mathrm{d} \hat{J}_{A}=0,
$$

so that $T^{4}$ is endowed with a hyper-Kähler structure. We will also assume that all the fields depend just on the $\left(u, v, x^{i}\right)$ coordinates along the six-dimensional space $\mathbb{R}^{1,1} \times Y$.

Finally we restrict our analysis to backgrounds that are 'isotropic' along $T^{4}$. In other words, we impose that $H$ has legs just along $\mathbb{R}^{1,1} \times Y$ and that the RR-flux polyform $F$ splits as follows:

$$
F_{\text {tot }}=F+\tilde{F} \wedge \widehat{\operatorname{vol}}_{4},
$$

where

$$
\begin{gathered}
F \equiv F_{1}+F_{3}+F_{5}, \\
\tilde{F} \equiv \tilde{F}_{1}+\tilde{F}_{3}+\tilde{F}_{5}
\end{gathered}
$$

have legs along $\mathbb{R}^{1,1} \times Y$ only, and $\widehat{\operatorname{vol}}_{4}$ is the volume form associated with the flat metric $\mathrm{d} \hat{s}_{4}^{2}$. To avoid confusion, in this section, we denote the ten-dimensional RR fluxes by the subscript tot The ten-dimensional self-duality condition $* \lambda\left(F_{\text {tot }}\right)=F_{\text {tot }}$ translates into the six-dimensional Hodge-duality

$$
\tilde{F}=e^{4 \hat{G}} *_{6} \lambda(F),
$$

where $*_{6}$ uses the complete, warped, six-dimensional metric. Notice that, by isotropy, $F$ and $\tilde{F}$ must satisfy the following six-dimensional Bianchi identities/equations of motion

$$
\begin{aligned}
& \mathrm{d}_{H} F=0, \\
& \mathrm{~d}_{H} \tilde{F}=0,
\end{aligned}
$$

where we recall that $\mathrm{d}_{H} \equiv \mathrm{d}-H$.

The BPS equations of Section 2.2.3 can be used to derive a general ansatz (provided the assumptions we made before) describing the D1-D5-P geometries we want to study. In particular the metric and fluxes can be expressed in terms of a reduced number of independent fields satisfying a simplified set of equations. The detailed derivation of such a minimal ansatz can be found in Appendix E. Here we simply discuss the main steps of that derivation and the final results, omitting several technical details.

Let us consider first (2.36a). As already discussed in Section 3.1, this can be used to derive the most general for of the NSNS two-form, under the assumption that $\mathcal{L}_{K} B=0$.

$$
B=-e^{2 D} \cos 2 \theta(\mathrm{d} u+\omega) \wedge(\mathrm{d} v+\beta)+b \wedge(\mathrm{d} v+\beta)+\mathcal{B},
$$

where $b=b_{i} \mathrm{~d} x^{i}$ and $\mathcal{B}=\frac{1}{2} \mathcal{B}_{i j} \mathrm{~d} x^{i} \wedge \mathrm{d} x^{j}$ have legs just along $Y$.

Similarly the 'isotropic' components of $(2.36 \mathrm{~b})$ can be used to determine the RR potentials. We define

$$
F=\mathrm{d}_{H} C, \quad \tilde{F}=\mathrm{d}_{H} \tilde{C},
$$

\footnotetext{
9 We stress once again that, with the two assumptions recalled after (3.26), everything we will say is actually valid for $\hat{Y}=\mathrm{K} 3$ and Ricci-flat $\mathrm{d} \hat{s}_{4}^{2} \equiv \mathrm{d} s_{\mathrm{K} 3}^{2}$ as well.
} 
where $C=C_{0}+C_{2}+C_{4}$ and $\tilde{C}=\tilde{C}_{0}+\tilde{C}_{2}+\tilde{C}_{4}$ are $u$-independent. Then the most general solution of (2.36b) for the potentials is

$$
\begin{aligned}
& C=-e^{2 D}(\mathrm{~d} u+\omega) \wedge(\mathrm{d} v+\beta) \wedge\left(e^{-\phi} \sin 2 \theta+\cos 2 \theta \mathcal{C}\right)+c \wedge(\mathrm{d} v+\beta)+\mathcal{C} \\
& \tilde{C}=-e^{2 D}(\mathrm{~d} u+\omega) \wedge(\mathrm{d} v+\beta) \wedge\left(e^{4 \hat{G}-\phi} \sin 2 \theta+\cos 2 \theta \tilde{\mathcal{C}}\right)+\tilde{c} \wedge(\mathrm{d} v+\beta)+\tilde{\mathcal{C}}
\end{aligned}
$$

where we have introduced the following polyforms on $Y$

$$
\begin{aligned}
& c \equiv c_{1}+c_{3}, \quad \tilde{c} \equiv \tilde{c}_{1}+\tilde{c}_{3}, \\
& \mathcal{C} \equiv \mathcal{C}_{0}+\mathcal{C}_{2}+\mathcal{C}_{4}, \quad \tilde{\mathcal{C}} \equiv \tilde{\mathcal{C}}_{0}+\tilde{\mathcal{C}}_{2}+\tilde{\mathcal{C}}_{4} .
\end{aligned}
$$

In order to proceed, we observe that the gauge freedom (3.2) can be conveniently fixed by imposing that

$$
e^{-2 G}=e^{2 D+2 \hat{G}-\phi} \sin 2 \theta .
$$

Then, the isotropy condition tells us the that the 'non-isotropic' components of (2.36b) must vanish. This gives conditions involving the two-forms $J_{A}$

$$
\mathcal{D} J_{A}-\dot{\beta} \wedge J_{A}=0
$$

and the one-form $\beta$

$$
{ }_{{ }_{4}} \mathcal{D} \beta=\mathcal{D} \beta \text {. }
$$

The first tells us that the non-trivial $v$-dependence of the background constitutes a potential obstruction to the integrability of the almost hyper-Kähler structure on $Y$. The second conditions is simply the self-duality of $\mathcal{D} \beta$.

By using (2.37a) and (2.37b) one can show that the dilaton is given by

$$
e^{\phi}=\frac{e^{2 \hat{G}}}{\sin 2 \theta},
$$

up to an arbitrary overall constant factor, which we have chosen to be 1 for simplicity.

It is convenient to explicitly solve the two relations (3.35) and (3.38) by expressing the three wrappings $e^{2 D}, e^{2 G}$ and $e^{2 \hat{G}}$, the angle $\theta$ and the dilaton $e^{\phi}$ in terms of three independent functions $Z, \tilde{Z}$ and $Z_{b}{ }^{10}$ :

$$
\begin{aligned}
& e^{2 D}=\frac{\alpha}{\sqrt{Z \tilde{Z}}}, \quad e^{2 G}=\sqrt{Z \tilde{Z}}, \quad e^{2 \hat{G}}=\sqrt{\frac{Z}{\tilde{Z}}}, \\
& \cos 2 \theta=\frac{Z_{b}}{\sqrt{Z \tilde{Z}}}, \quad e^{2 \phi}=\alpha \frac{Z}{\tilde{Z}},
\end{aligned}
$$

where we have introduced a further function

$$
\alpha \equiv \frac{Z \tilde{Z}}{Z \tilde{Z}-Z_{b}^{2}}=\frac{1}{\sin ^{2} 2 \theta} .
$$

10 It can be useful to list the inverse relations too: $Z=e^{2 G+2 \hat{G}}, \tilde{Z}=e^{2 G-2 \hat{G}}$ and $Z_{b}=\cos 2 \theta e^{2 G}$, with $e^{2 G}$ given by (3.35). 
By combining (2.37a) and (2.37b) with the self-duality condition for the RR field-strengths we can also fix

$$
\mathcal{C}_{0}=\frac{Z_{b}}{Z}, \quad \tilde{\mathcal{C}_{0}}=\frac{Z_{b}}{\tilde{Z}},
$$

up to an additive constant, which we set equal to zero. We then see that part of the supersymmetry conditions can be used to fix the form of all scalars in our ansatz in terms of just the three functions $Z, \tilde{Z}$ and $Z_{b}$.

In order to write the remaining supersymmetry conditions in a more inspiring form, let us introduce the anti-self-dual two form [38]

$$
\psi \equiv \frac{1}{8} \epsilon^{A B C}\left(J_{A}\right)^{i j}\left(\dot{J}_{B}\right)_{i j} J_{C},
$$

which measures the rotation of the triplet $\left\{J_{A}\right\}$ under the $v$-flow, and a set of three two-forms

$$
\begin{aligned}
& \Theta=\dot{\mathcal{C}}_{2}+\mathcal{D} c_{1}-\dot{\beta} \wedge c_{1}, \\
& \tilde{\Theta}=\dot{\tilde{\mathcal{C}}}_{2}+\mathcal{D} \tilde{c}_{1}-\dot{\beta} \wedge \tilde{c}_{1}, \\
& \Theta_{b}=\dot{\mathcal{B}}+\mathcal{D} b-\dot{\beta} \wedge b .
\end{aligned}
$$

Then, the self-duality of the RR fields implies that the anti-self-dual components of $\Theta, \tilde{\Theta}$ and $\Theta_{b}$ are proportional to $\psi$

$$
\begin{aligned}
& \left(1-*_{4}\right) \Theta=2 \tilde{Z} \psi, \\
& \left(1-*_{4}\right) \tilde{\Theta}=2 Z \psi, \\
& \left(1-*_{4}\right) \Theta_{b}=2 Z_{b} \psi,
\end{aligned}
$$

and determines the self-dual component of $\mathcal{D} \omega$

$$
\mathcal{D} \omega+*_{4} \mathcal{D} \omega=Z *_{4} \Theta+\tilde{Z} \tilde{\Theta}-Z_{b}\left(\Theta_{b}+*_{4} \Theta_{b}\right)-2 W \mathcal{D} \beta .
$$

Notice that the self-duality of the r.h.s. of (3.45) is guaranteed by (3.44a) and (3.44b).

The remaining conditions encoded in the self-duality of the RR fields can be shown to reduce to other two sets of equations. The first set is

$$
\begin{aligned}
& \mathcal{D} \mathcal{C}_{2}-\mathcal{D} \beta \wedge c_{1}=*_{4}(\mathcal{D} \tilde{Z}+\tilde{Z} \dot{\beta}), \\
& \mathcal{D} \tilde{\mathcal{C}}_{2}-\mathcal{D} \beta \wedge \tilde{c}_{1}=*_{4}(\mathcal{D} Z+Z \dot{\beta}), \\
& \mathcal{D B}-\mathcal{D} \beta \wedge b=*_{4}\left(\mathcal{D} Z_{b}+Z_{b} \dot{\beta}\right),
\end{aligned}
$$

and the second set is

$$
\begin{aligned}
& \dot{\mathcal{C}}_{4}+\mathcal{D} c_{3}-\dot{\beta} \wedge c_{3}-\Theta_{b} \wedge \mathcal{C}_{2}-(\mathcal{D B}-\mathcal{D} \beta \wedge b) \wedge c_{1}=\tilde{Z}^{2} \frac{\mathrm{d}}{\mathrm{d} v}\left(\frac{Z_{b}}{\tilde{Z}}\right) \mathrm{vol}_{4}, \\
& \dot{\tilde{\mathcal{C}}}_{4}+\mathcal{D} \tilde{c}_{3}-\dot{\beta} \wedge \tilde{c}_{3}-\Theta_{b} \wedge \tilde{\mathcal{C}}_{2}-(\mathcal{D B}-\mathcal{D} \beta \wedge b) \wedge \tilde{c}_{1}=Z^{2} \frac{\mathrm{d}}{\mathrm{d} v}\left(\frac{Z_{b}}{Z}\right) \mathrm{vol}_{4} .
\end{aligned}
$$

In fact, this latter set of equations does not give any additional constraint since it can always be solved locally: one can always choose a gauge in which $\mathcal{C}_{4} \equiv \tilde{\mathcal{C}}_{4}=0$ and Eqs. (3.47) can always be (locally) integrated to give the (local) expression for $c_{3}$ and $\tilde{c}_{3}$. 
To conclude this section it might be useful to summarise what we obtain for the different gauge invariant fields once we have implemented the above constraints. The metric ansatz (3.1) can be rewritten as

$$
\mathrm{d} s_{(10)}^{2}=-\frac{2 \alpha}{\sqrt{Z \tilde{Z}}}(\mathrm{~d} v+\beta)[\mathrm{d} u+\omega+W(\mathrm{~d} v+\beta)]+\sqrt{Z \tilde{Z}} \mathrm{~d} s_{4}^{2}+\sqrt{\frac{Z}{\tilde{Z}}} \mathrm{~d} \hat{s}_{4}^{2} .
$$

The dilaton is given by

$$
e^{2 \phi}=\alpha \frac{Z}{\tilde{Z}}
$$

The NSNS field-strength is

$$
\begin{aligned}
H= & -(\mathrm{d} u+\omega) \wedge(\mathrm{d} v+\beta) \wedge\left[\mathcal{D}\left(\frac{\alpha Z_{b}}{Z \tilde{Z}}\right)-\frac{\alpha Z_{b}}{Z \tilde{Z}} \dot{\beta}\right] \\
& +(\mathrm{d} v+\beta) \wedge\left(\Theta_{b}-\frac{\alpha Z_{b}}{Z \tilde{Z}} \mathcal{D} \omega\right)+\frac{\alpha Z_{b}}{Z \tilde{Z}}(\mathrm{~d} u+\beta) \wedge \mathcal{D} \beta+*_{4}\left(\mathcal{D} Z_{b}+Z_{b} \dot{\beta}\right) .
\end{aligned}
$$

The RR field-strengths (3.28a) are

$$
\begin{aligned}
F_{1}= & \mathcal{D}\left(\frac{Z_{b}}{Z}\right)+(\mathrm{d} v+\beta) \wedge \frac{\mathrm{d}}{\mathrm{d} v}\left(\frac{Z_{b}}{Z}\right), \\
F_{3}= & -(\mathrm{d} u+\omega) \wedge(\mathrm{d} v+\beta) \wedge\left[\mathcal{D}\left(\frac{1}{Z}\right)-\frac{1}{Z} \dot{\beta}+\frac{\alpha Z_{b}}{Z \tilde{Z}} \mathcal{D}\left(\frac{Z_{b}}{Z}\right)\right] \\
& +(\mathrm{d} v+\beta) \wedge\left(\Theta-\frac{Z_{b}}{Z} \Theta_{b}-\frac{1}{Z} \mathcal{D} \omega\right)+\frac{1}{Z}(\mathrm{~d} u+\omega) \wedge \mathcal{D} \beta \\
& +*_{4}(\mathcal{D} \tilde{Z}+\tilde{Z} \dot{\beta})-\frac{Z_{b}}{Z} *_{4}\left(\mathcal{D} Z_{b}+Z_{b} \dot{\beta}\right), \\
F_{5}= & -\frac{\alpha}{Z}(\mathrm{~d} u+\omega) \wedge(\mathrm{d} v+\beta) \wedge *_{4}\left[\frac{Z_{b}}{\tilde{Z}}(\mathcal{D} \tilde{Z}+\tilde{Z} \dot{\beta})-\mathcal{D} Z_{b}-Z_{b} \dot{\beta}\right] \\
& +\tilde{Z}^{2} \frac{\mathrm{d}}{\mathrm{d} v}\left(\frac{Z_{b}}{\tilde{Z}}\right)(\mathrm{d} v+\beta) \wedge \operatorname{vol}_{4},
\end{aligned}
$$

while the RR field-strength (3.28b) are given by

$$
\begin{aligned}
\tilde{F}_{1}= & \mathcal{D}\left(\frac{Z_{b}}{\tilde{Z}}\right)+(\mathrm{d} v+\beta) \wedge \frac{\mathrm{d}}{\mathrm{d} v}\left(\frac{Z_{b}}{\tilde{Z}}\right), \\
\tilde{F}_{3}= & -(\mathrm{d} u+\omega) \wedge(\mathrm{d} v+\beta) \wedge\left[\mathcal{D}\left(\frac{1}{\tilde{Z}}\right)-\frac{1}{\tilde{Z}} \dot{\beta}+\frac{\alpha Z_{b}}{Z \tilde{Z}} \mathcal{D}\left(\frac{Z_{b}}{\tilde{Z}}\right)\right] \\
& +(\mathrm{d} v+\beta) \wedge\left(\tilde{\Theta}-\frac{Z_{b}}{\tilde{Z}} \Theta_{b}-\frac{1}{\tilde{Z}} \mathcal{D} \omega\right)+\frac{1}{\tilde{Z}}(\mathrm{~d} u+\omega) \wedge \mathcal{D} \beta \\
& +*_{4}(\mathcal{D} Z+Z \dot{\beta})-\frac{Z_{b}}{\tilde{Z}} *_{4}\left(\mathcal{D} Z_{b}+Z_{b} \dot{\beta}\right), \\
\tilde{F}_{5}= & -\frac{\alpha}{\tilde{Z}}(\mathrm{~d} u+\omega) \wedge(\mathrm{d} v+\beta) \wedge *_{4}\left[\frac{Z_{b}}{Z}(\mathcal{D} Z+Z \dot{\beta})-\mathcal{D} Z_{b}-Z_{b} \dot{\beta}\right] \\
& +Z^{2} \frac{\mathrm{d}}{\mathrm{d} v}\left(\frac{Z_{b}}{Z}\right)(\mathrm{d} v+\beta) \wedge \operatorname{vol}_{4} .
\end{aligned}
$$


Our general supersymmetric ansatz is completely specified in terms of the fields $\mathrm{d} s_{4}^{2}, \omega, \beta, W, Z$, $\tilde{Z}, Z_{b}, \Theta, \tilde{\Theta}$ and $\Theta_{b}$. They have to satisfy (3.36), (3.37), (3.44), (3.45) and (3.46), which ensure the Bianchi identities for the NSNS and RR fields, as well as the RR self-duality condition (3.29). ${ }^{11}$ Notice that the definitions (3.43) and the conditions (3.46), which explicitly involve some (locally defined) RR and NSNS potentials, can be substituted with the conditions

$$
\begin{aligned}
& \mathcal{D} \Theta-\dot{\beta} \wedge \Theta=\frac{\mathrm{d}}{\mathrm{d} v} *_{4}(\mathcal{D} \tilde{Z}+\tilde{Z} \dot{\beta}), \\
& \mathcal{D} \tilde{\Theta}-\dot{\beta} \wedge \tilde{\Theta}=\frac{\mathrm{d}}{\mathrm{d} v} *_{4}(\mathcal{D} Z+Z \dot{\beta}), \\
& \mathcal{D} \Theta_{b}-\dot{\beta} \wedge \Theta_{b}=\frac{\mathrm{d}}{\mathrm{d} v} *_{4}\left(\mathcal{D} Z_{b}+Z_{b} \dot{\beta}\right),
\end{aligned}
$$

and

$$
\begin{aligned}
& \mathcal{D} *_{4}(\mathcal{D} Z+\dot{\beta} Z)=-\tilde{\Theta} \wedge \mathcal{D} \beta, \\
& \mathcal{D} *_{4}(\mathcal{D} \tilde{Z}+\dot{\beta} \tilde{Z})=-\Theta \wedge \mathcal{D} \beta, \\
& \mathcal{D} *_{4}\left(\mathcal{D} Z_{b}+\dot{\beta} Z_{b}\right)=-\Theta_{b} \wedge \mathcal{D} \beta,
\end{aligned}
$$

respectively. Indeed, (3.43) and (3.46) can be regarded as explicit local solutions of Eqs. (3.53) and (3.54).

As explained in Section 2.2.2, in order to obtain a supersymmetry solution, one needs to further impose the $\underline{v v}$ component of the Einstein equation (2.38). By using the parametrisation introduced in this section and some of the above constraints imposed by supersymmetry, this reduces to the following equation

$$
\begin{aligned}
*_{4} \mathcal{D} & *_{4} L+2 \dot{\beta}_{i} L^{i}-\frac{1}{4} \frac{Z \tilde{Z}}{\alpha}\left[\frac{\tilde{Z}}{Z} \frac{\mathrm{d}}{\mathrm{d} v}\left(\frac{Z}{\tilde{Z}}\right)\right]^{2} \\
- & \frac{\alpha}{4} \frac{\mathrm{d}}{\mathrm{d} v}\left(\frac{\sqrt{Z \tilde{Z}}}{\alpha^{2}} g^{i j}\right) \frac{\mathrm{d}}{\mathrm{d} v}\left(\sqrt{Z \tilde{Z}} g_{i j}\right)-\frac{1}{2} \frac{\sqrt{Z \tilde{Z}}}{\alpha} g^{i j} \frac{\mathrm{d}^{2}}{\mathrm{~d} v^{2}}\left(\sqrt{Z \tilde{Z}} g_{i j}\right) \\
+ & \sqrt{Z \tilde{Z}} \frac{\mathrm{d}}{\mathrm{d} v}\left[\sqrt{Z \tilde{Z}_{2}} \frac{\mathrm{d}}{\mathrm{d} v}\left(\frac{Z_{b}^{2}}{Z \tilde{Z}}\right)\right]-\frac{1}{2} Z^{2}\left[\frac{\mathrm{d}}{\mathrm{d} v}\left(\frac{Z_{b}}{Z}\right)\right]^{2}-\frac{1}{2} \tilde{Z}^{2}\left[\frac{\mathrm{d}}{\mathrm{d} v}\left(\frac{Z_{b}}{\tilde{Z}}\right)\right]^{2} \\
+ & \frac{1}{2} *_{4}\left[(\Theta-\tilde{Z} \psi) \wedge(\tilde{\Theta}-Z \psi)-\left(\Theta_{b}-Z_{b} \psi\right) \wedge\left(\Theta_{b}-Z_{b} \psi\right)\right. \\
+ & \left.\frac{Z \tilde{Z}}{\alpha} \psi \wedge \psi-2 \psi \wedge \mathcal{D} \omega\right]=0,
\end{aligned}
$$

where we recall that

$$
L=\dot{\omega}+W \dot{\beta}-\mathcal{D} W,
$$

and $g_{i j}$ are the components of the metric on $Y: \mathrm{d} s_{4}^{2}=g_{i j} \mathrm{~d} x^{i} \mathrm{~d} x^{j}$.

It is useful to examine some limits of the general ansatz we have found above to clarify how previously known solutions embed in it. When the metric and the gauge fields are taken to be independent of $v$, the solution reduces to the one of $[44,45]$ and it can be reduced to $\mathcal{N}=25 \mathrm{D}$

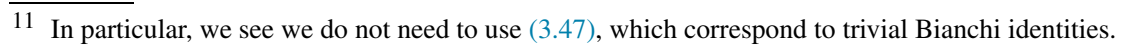


supergravity coupled to three vector multiplets. When $Z_{b}=b=\mathcal{B}=0$ one re-obtains the ansatz of $[39,40]$, which is equivalent to 6D supergravity coupled to an anti-self-dual tensor multiplet (if one further restricts $Z=\tilde{Z}, c_{1}=\tilde{c}_{1}, \mathcal{C}_{2}=\tilde{\mathcal{C}}_{2}$, one reduces to minimal $6 \mathrm{D}$ supergravity, studied in [38]).

We would like to conclude this section by emphasising that, in order to find explicit solutions, the minimal set of equations we listed above can be conveniently organised in a way which highlights a hidden linear structure [40]. This is discussed in Appendix E.7.

\subsection{Supersymmetry of the solution}

The supergravity backgrounds described in Section 3.2 are 1/8 supersymmetric, that is they preserve four supercharges, both for $\hat{Y}=T^{4}$ or K3. In order to understand this point we need to count the number of independent degrees of freedom of the spinors $\epsilon_{1}$ and $\epsilon_{2}$ satisfying the supersymmetry conditions. According to our ansatz (3.7) and (3.14)-(3.17) these are given by

$$
\epsilon_{I}=N_{I}\left(\begin{array}{l}
1 \\
0
\end{array}\right) \otimes\left(\zeta \otimes \hat{\zeta}+\zeta^{\mathrm{c}} \otimes \hat{\zeta}^{\mathrm{c}}\right),
$$

where the spinors $\zeta$ and $\hat{\zeta}$ are positive chirality spinors of unitary norm on $Y$ and $T^{4}$, respectively. The functions $N_{I}$ are given in (3.14): $N_{1}=2^{1 / 4} \sin \theta$ and $N_{2}=2^{1 / 4} \cos \theta$.

In our approach we trade spinors for forms built as bilinears in the spinors. Indeed, the complete information on the spinorial ansatz (3.57) is carried by the angle $\theta$ and the four-form $\Omega$ introduced in (3.19). In turn, the information on $\zeta$ and $\hat{\zeta}$ is encoded in the two almost hyper-Kähler structures $\left(J_{1}, J_{2}, J_{3}\right)$ and $\left(\hat{J}_{1}, \hat{J}_{2}, \hat{J}_{3}\right)$ (see $\left.(3.21)\right)$, which enter $\Omega$ through the combination

$$
\sum_{A} J_{A} \wedge \hat{J}_{A} .
$$

Now, it is immediate to check that all the equations for our backgrounds are invariant under separate rigid $\mathrm{SU}(2) \simeq \mathrm{SO}(3)$ rotations of $J_{A}$ and $\hat{J}_{A}$, say $\mathrm{SU}(2) \times \widehat{\mathrm{SU}}(2)$. Such transformations do not change the metric and the other bosonic fields but do generically transform the form $\Omega$, which is left invariant only under by the diagonal subgroup $\mathrm{SU}(2)_{\operatorname{diag}} \subset \mathrm{SU}(2) \times \widehat{\mathrm{SU}}(2)$ which preserves the combination (3.58). ${ }^{12}$ This means that these rigid $\mathrm{SU}(2) \times \widehat{\mathrm{SU}}(2)$ transformations produce a three-parameters family of inequivalent normalised Killing spinors, locally identifiable with the coset $[\mathrm{SU}(2) \times \widehat{\mathrm{SU}}(2)] / \mathrm{SU}(2)_{\text {diag. }}$. We also have the freedom of a constant rescaling of the Killing spinors. If we take into account this extra parameter, we obtain a four-parameter family of Killing spinors, corresponding to four supercharges preserved by our backgrounds.

\section{An exact solution}

The supergravity analysis of the previous sections provides a general framework for the construction of supersymmetric type IIB solutions with a null isometry. In particular it can be specialised to the study of type IIB backgrounds containing a $T^{4}$ and isotropic in $T^{4}$. The main physical application that motivated this analysis is the construction of the generic solution carrying D1, D5 and P charges. As mentioned in the Introduction, it would be very interesting to see

$\overline{12}$ The SU(2) rotations translate into analogous rotations of the spinors $\zeta$ and $\hat{\zeta}$, and again it is easy to see that (3.57) is left invariant only by the diagonal subgroup $\mathrm{SU}(2)_{\mathrm{diag}}$. 
whether the subset of D1-D5-P microstates that are isotropic in $T^{4}$ is sufficient to account for a finite fraction of the total entropy associated with the given asymptotic charges.

In this section we present the first example of a configuration of this kind: an exact, completely regular, horizonless, $v$-dependent solution of the supergravity equations of Section 2.2.3 carrying D1, D5 and P charges, with a non-trivial profile for all type IIB fields. It also admits an AdS limit which is dual to a known state of the D1-D5 CFT. Our main purpose is to show that it is possible to find such explicit $v$-dependent solutions, ${ }^{13}$ rather than to discuss how to build them. That is why we will give the explicit form of the solution but only briefly sketch the method that was used to construct it (this is basically the approach of [50], just implemented at the full non-linear level). A more detailed presentation of the solution generating technique, of the regularity analysis and of the duality with the D1-D5 CFT, together with possible generalisations, will be presented in a forthcoming work.

The solution we obtain can be seen as the non-linear completion of an approximate solution derived by Mathur, Saxena and Srivastava (MSS) in [42], which represented the first example of a microstate geometry for the three-charge black hole. We will review below the approximate construction of [42], and then outline our method to promote that solution to an exact one of the non-linear supergravity equations.

Note

To make contact with the existing literature on D1-D5-P microstates, in this section we will use a different notation than in the previous part of the paper. The switch of notation and the supergravity equations in the new variables are give in Appendix E.7. From now on we will refer to equations in that appendix.

\subsection{The perturbative solutions of MSS}

The three-charge solution of MSS [42] is realised as a perturbation around a particular two-charge background. Thus we begin with a very brief summary of the D1-D5 1/4-BPS microstates. These geometries were derived starting from the solution describing the back-reaction of a vibrating F1-string [51,52]. When the asymptotic 10D geometry is $\mathbb{R}^{1,4} \times S^{1} \times T^{4}$ it is possible to perform a U-duality transformation mapping the F1-P charges into the D1-D5 charges. One obtains a microstate defined by a curve $g_{i}\left(v^{\prime}\right)$ in $\mathbb{R}^{4}$. We are interested in the Lunin-Mathur solution $[53,54]$ which is defined by the circular profile

$$
g_{1}\left(v^{\prime}\right)=a \cos \frac{2 \pi v^{\prime}}{L}, \quad g_{2}\left(v^{\prime}\right)=a \cos \frac{2 \pi v^{\prime}}{L}, \quad g_{3}\left(v^{\prime}\right)=g_{4}\left(v^{\prime}\right)=0 .
$$

Here

$$
a=\frac{\sqrt{Q_{1} Q_{5}}}{R}, \quad L=\frac{2 \pi Q_{5}}{R},
$$

where $R$ is the radius of the direction $y$ common to the D1 and D5 branes, and $Q_{1}$ and $Q_{5}$ are the charges for $N_{1}$ D1 and $N_{5}$ D5 branes. The $v^{\prime}$ appearing in the equation above has to be thought as

\footnotetext{
13 The example discussed in [36] falls in the restricted ansatz of [40] and a full analysis of the regularity conditions of this case has still to be performed. Another very interesting family of a $v$-dependent geometries has been recently discussed in [46,47]; these solutions are somehow complementary to those discussed in this paper as they are non-trivial along $T^{4}$ and are identical to the two-charge configurations for the $6 \mathrm{D}$ part. A class of $v$-dependent but unbound solutions has been found in $[48,49]$.
} 
a parameter along the profile $g_{i}\left(v^{\prime}\right)$, and is not to be confused with the space-time coordinates $v$, used in the supergravity sections, which is related to the time coordinate $t$ and the $S^{1}$ coordinate $y$ as in (2.14).

In this and the next subsection we will restrict, as in [42], to the particular case in which $Q_{1}=Q_{5}=Q$. This ensures that the 6D description of the solution in (4.3a)-(4.3d) is simpler as it can be described by minimal supergravity. In this case, the Lunin-Mathur geometry with circular profile can be written in terms of the ansatz (E.51) as follows:

$$
\begin{aligned}
& \mathrm{d} s_{4}^{2}=\left(r^{2}+a^{2} \cos ^{2} \theta\right)\left(\frac{\mathrm{d} r^{2}}{r^{2}+a^{2}}+\mathrm{d} \theta^{2}\right)+\left(r^{2}+a^{2}\right) \sin ^{2} \theta \mathrm{d} \phi^{2}+r^{2} \cos ^{2} \theta \mathrm{d} \psi^{2}, \\
& \beta=\frac{R a^{2}}{\sqrt{2}\left(r^{2}+a^{2} \cos ^{2} \theta\right)}\left(\sin ^{2} \theta \mathrm{d} \phi-\cos ^{2} \theta \mathrm{d} \psi\right), \\
& Z_{1}=Z_{2}=1+\frac{Q}{r^{2}+a^{2} \cos ^{2} \theta}, \quad a_{1}=a_{2}=0, \\
& \omega=\frac{R a^{2}}{\sqrt{2}\left(r^{2}+a^{2} \cos ^{2} \theta\right)}\left(\sin ^{2} \theta \mathrm{d} \phi+\cos ^{2} \theta \mathrm{d} \psi\right), \quad \mathcal{F}=0, \\
& Z_{4}=a_{4}=\delta_{2}=0 .
\end{aligned}
$$

Note that the $4 \mathrm{D}$ base metric $\mathrm{d} s_{4}^{2}$ in (4.3a) is just flat $\mathbb{R}^{4}$ written in non-standard coordinates, in which the 10D metric takes its simplest form.

For $r \sim \sqrt{Q}$ a curved region of space-time, named the "throat", opens up. Contrary to the "naive" extremal black hole geometry (which corresponds to the case $a=0$ ), the throat ends smoothly after a coordinate distance of order $Q / a$. As usual, the decoupling (or "near-horizon") limit corresponds to the case in which one focuses on the region inside a throat whose length is much larger than its width. Quantitatively this approximation requires

$$
r \ll \sqrt{Q}, \quad a \ll \sqrt{Q} .
$$

In this limit, the " 1 " in the expression (4.3c) for $Z_{1}$ and $Z_{2}$ can be neglected:

$$
Z_{1}^{\mathrm{nh}}=Z_{2}^{\mathrm{nh}}=\frac{Q}{r^{2}+a^{2} \cos ^{2} \theta},
$$

while all the other geometric data are left unchanged. One can see that the resulting 10D geometry reduces to $A d S_{3} \times S^{3} \times T^{4}$ after the coordinate redefinition

$$
\phi \rightarrow \phi+\frac{t}{R}, \quad \psi \rightarrow \psi+\frac{y}{R} .
$$

According to the general AdS/CFT paradigm, the full string (or M) theory in an AdS space arising from a "near-horizon" limit should be dual to the CFT describing the low-energy approximation of the theory living on the branes used in the construction. In our case, we have to deal with the $(1+1)$-dimensional CFT with central charge $6 N_{1} N_{5}[55,56]$ that captures the low energy dynamics of the open strings ending on the D1 and D5 branes. Let us recall that this CFT has an $\mathrm{SU}(2)_{L} \times \mathrm{SU}(2)_{R}$ R-symmetry, corresponding to rotations of $\mathbb{R}^{4}$, whose affine generators are $J_{n}^{3}, J_{n}^{ \pm}$and $\bar{J}_{n}^{3}, \bar{J}_{n}^{ \pm}$. According to the standard AdS/CFT dictionary, the "empty" $A d S_{3} \times S^{3} \times T^{4}$ space corresponds to the vacuum state of the CFT, which is in the NSNS sector, while the geometry defined by Eq. (4.1) is dual to the RR ground state of the D1-D5 CFT with maximal values of $J_{0}^{3}$ and $\bar{J}_{0}^{3}$ (i.e. the highest weight state in each $\mathrm{SU}(2)$ given the total angular momentum of the geometry). Then, from the point of view of the CFT, the coordinate change 
(4.6) realises the spectral flow from the RR sector to the NSNS sector [57], since it connects the geometry dual to a RR ground state to the "empty" $A d S_{3} \times S^{3} \times T^{4}$, dual to the NSNS ground state.

Non-trivial chiral primaries of the CFT are more easily described by reducing to sixdimensional supergravity, and are represented by supergravity perturbations of the $A d S_{3} \times S^{3}$ background. This is the point of view adopted by the authors of [42], who consider a $6 \mathrm{D}$ supergravity comprising the gravity multiplet (whose bosonic part contains the metric, a self-dual 2-form $C$ and a vector) and a tensor multiplet (which includes an "anti-self-dual" 2-form $B$, a scalar $w$ and a vector). The metric and $C$ describe the $A d S_{3} \times S^{3}$ background:

$$
\begin{aligned}
& \mathrm{d} s_{6}^{2}=-\frac{r^{2}+a^{2}}{Q} \mathrm{~d} t^{2}+\frac{r^{2}}{Q} \mathrm{~d} y^{2}+Q \frac{\mathrm{d} r^{2}}{r^{2}+a^{2}}+Q\left(\mathrm{~d} \theta^{2}+\cos ^{2} \theta \mathrm{d} \psi^{2}+\sin ^{2} \theta \mathrm{d} \phi^{2}\right), \\
& C=-\frac{r^{2}+a^{2}}{Q} \mathrm{~d} t \wedge \mathrm{d} y-Q \cos ^{2} \theta \mathrm{d} \phi \wedge \mathrm{d} \psi .
\end{aligned}
$$

Then, following [42], we switch on a perturbation that sits in the tensor multiplet and only excites the fields $B$ and $w$. The equations satisfied by $B$ and $w$ are the $6 \mathrm{D}$ supergravity equations linearised around the $A d S_{3} \times S^{3}$ background and are given by

$$
\mathrm{d} B+*_{6} \mathrm{~d} B+w \mathrm{~d} C=0, \quad \mathrm{~d} *_{6} \mathrm{~d} w-2 \mathrm{~d} B \wedge \mathrm{d} C=0 .
$$

The explicit form of the perturbation ${ }^{14}$ is [42]

$$
\begin{aligned}
w= & \frac{c_{l}}{Q} e^{-2 i l\left(\phi+\frac{t}{R}\right)} \frac{\sin ^{2 l} \theta}{\left(r^{2}+a^{2}\right)^{l}}, \\
B= & \frac{c_{l}}{2} e^{-2 i l\left(\phi+\frac{t}{R}\right)} \frac{\sin ^{2 l} \theta}{\left(r^{2}+a^{2}\right)^{l}}\left[-\frac{r^{2}}{Q^{2}} \mathrm{~d} t \wedge \mathrm{d} y-\frac{i}{R} \frac{r}{r^{2}+a^{2}} \mathrm{~d} r \wedge \mathrm{d} y\right. \\
& \left.-\cos ^{2} \theta \mathrm{d} \phi \wedge \mathrm{d} \psi-i \frac{\cos \theta}{\sin \theta} \mathrm{d} \theta \wedge \mathrm{d} \psi\right] .
\end{aligned}
$$

This perturbation is dual to a chiral primary state $|\Psi\rangle_{N S}$ identified by the quantum numbers

$$
j_{N S}=h_{N S}=l, \quad \bar{j}_{N S}=\bar{h}_{N S}=l,
$$

where $j_{N S}$ and $\bar{j}_{N S}$ are the eigenvalues of $J_{0}^{3}$ and $\bar{J}_{0}^{3}$ and $h_{N S}$ and $\bar{h}_{N S}$ are the eigenvalues of the Virasoro generators $L_{0}$ and $\bar{L}_{0}$.

For later convenience, it is also useful to analyse the corresponding solution in the RR sector. $|\Psi\rangle_{N S}$ maps, via the inverse of the spectral flow transformation

$$
\phi \rightarrow \phi-\frac{t}{R}, \quad \psi \rightarrow \psi-\frac{y}{R},
$$

into a RR ground state $|\Psi\rangle_{R}$ with $^{15}$

$$
j_{R}=l, \quad h_{R}=0, \quad \bar{j}_{R}=l, \quad \bar{h}_{R}=0 .
$$

\footnotetext{
14 In all linearised solutions we follow [42] and complexify the field describing the perturbation: both the real and the imaginary parts of this field represent valid solutions. Of course, at the non-linear level we will always have to work with real fields.

15 Spectral flow transforms the CFT quantum numbers as $h_{R}=h_{N S}-j_{N S}+\frac{c}{24}, j_{R}=j_{N S}-\frac{c}{12}$, with $c=6 N_{1} N_{5}$ for the D1-D5 CFT. The terms proportional to $c$ are associated with the background and thus, for the perturbation alone, one has $h_{R}=h_{N S}-j_{N S}, j_{R}=j_{N S}$.
} 
The geometry corresponding to $|\Psi\rangle_{R}$ is represented as a perturbation around the background given in Eqs. (4.3a), (4.3b), (4.3d) and (4.5); the fields of the perturbation ${ }^{16}$ read

$$
\begin{aligned}
w= & \frac{c_{l}}{Q} e^{-2 i l \phi} \frac{\sin ^{2 l} \theta}{\left(r^{2}+a^{2}\right)^{l}}, \\
B= & \frac{c_{l}}{2} e^{-2 i l \phi} \frac{\sin ^{2 l} \theta}{\left(r^{2}+a^{2}\right)^{l}}\left[-\frac{r^{2}+a^{2} \cos ^{2} \theta}{Q^{2}} \mathrm{~d} t \wedge \mathrm{d} y-\frac{i}{R} \frac{r}{r^{2}+a^{2}} \mathrm{~d} r \wedge \mathrm{d} y\right. \\
& -\cos ^{2} \theta\left(\mathrm{d} \phi \wedge \mathrm{d} \psi-\frac{a}{Q}(\mathrm{~d} t \wedge \mathrm{d} \psi+\mathrm{d} \phi \wedge \mathrm{d} y)\right) \\
& \left.-i \frac{\cos \theta}{\sin \theta}\left(\mathrm{d} \theta \wedge \mathrm{d} \psi-\frac{a}{Q} \mathrm{~d} \theta \wedge \mathrm{d} y\right)\right] .
\end{aligned}
$$

The idea of [42] is that, by a sequence of transformations in the chiral algebra of the CFT, $|\Psi\rangle_{N S}$ (and so the RR state $|\Psi\rangle_{R}$ obtained after an inverse spectral flow) can be related to a state in the RR sector of the CFT carrying one unit of momentum. In particular, one can consider the state $J_{0}^{-}|\Psi\rangle_{N S}$ : it has

$$
j_{N S}=l-1, \quad h_{N S}=l, \quad \bar{j}_{N S}=\bar{h}_{N S}=l,
$$

and hence is not a chiral primary. If one performs an inverse spectral flow transformation one then reaches a RR state which is not a ground state and whose quantum numbers are

$$
j_{R}=l-1, \quad h_{R}=1, \quad \bar{j}_{R}=l, \quad \bar{h}_{R}=0 .
$$

This state can be identified with $J_{-1}^{-}|\Psi\rangle_{R}$; it carries momentum

$$
p=h_{R}-\bar{h}_{R}=1 \text {. }
$$

Thanks to the fact that the operator $J_{0}^{-}$can be identified with an infinitesimal rotation in $\mathbb{R}^{4}$, it is straightforward to generate (in the near-horizon limit) the gravity solution dual of the state $J_{-1}^{-}|\Psi\rangle_{R}$ : one starts with the solution (4.9a)-(4.9b), performs the infinitesimal rotation associated with $J_{0}^{-}$, and finally the change of coordinates (4.11). The resulting geometry, whose explicit expression can be found in Eqs. (3.21)-(3.30) of [42], solves by construction the linearised equations in the "near-horizon" region. In order to construct a real microstate of the three-charge black hole, this "near-horizon" geometry should be glued back to the asymptotically flat region. This step was performed in [42] only approximately, through a perturbative expansion in the parameter $\epsilon=\frac{a}{\sqrt{Q}}$ (the regime $\epsilon \ll 1$ describes geometries with a very long throat). In the next subsection we will show that, by embedding the solution into our general ansatz (E.51), the extension from the asymptotically AdS solution to an asymptotically flat one is straightforward. The same formalism will also make the generalisation from a linearised to an exact background more transparent.

\subsection{Embedding MSS in our ansatz}

Let us go back to the original two-charge geometry (4.13a)-(4.13b) corresponding to the CFT state in the RR sector $|\Psi\rangle_{R}$ : to embed this 6D supergravity solution into our 10D ansatz one first

$\overline{16}$ In order to obtain (4.13b) one needs to use also $Q=R a$ following from (4.2) in the case $Q_{1}=Q_{5}$. 
needs to specify a 10D uplift. This uplift is not unique, but we focus here on one based on the following identifications ${ }^{17}$

$$
C \equiv-C_{2}, \quad w \equiv 2 C_{0}, \quad B \equiv B .
$$

With these identifications, the state $|\Psi\rangle_{R}$ is represented by the original Lunin-Mathur geometry given in (4.3a), (4.3b), (4.3d) and (4.5), plus the 10D uplift of the perturbation Eqs. (4.13a)-(4.13b):

$$
\begin{aligned}
C_{0}= & \frac{c_{l}}{2 Q} e^{-2 i l \phi} \frac{\sin ^{2 l} \theta}{\left(r^{2}+a^{2}\right)^{l}}, \\
B= & \frac{c_{l}}{2}\left\{e ^ { - 2 i l \phi } \frac { \operatorname { s i n } ^ { 2 l } \theta } { ( r ^ { 2 } + a ^ { 2 } ) ^ { l } } \left[-\frac{r^{2}+a^{2} \cos ^{2} \theta}{Q^{2}}(\mathrm{~d} u+\omega) \wedge(\mathrm{d} v+\beta)\right.\right. \\
& \left.-\frac{r^{2}+a^{2}}{r^{2}+a^{2} \cos ^{2} \theta} \cos ^{2} \theta \mathrm{d} \phi \wedge \mathrm{d} \psi-i \frac{\cos \theta}{\sin \theta} \mathrm{d} \theta \wedge \mathrm{d} \psi\right] \\
& \left.+e^{-2 i l \phi} \frac{\sin ^{2 l} \theta}{\left(r^{2}+a^{2}\right)^{l}}\left[\frac{a}{Q}\left(\mathrm{~d} \phi+i \frac{\cos \theta}{\sin \theta} \mathrm{d} \theta-i \frac{r}{r^{2}+a^{2}} \mathrm{~d} r\right) \wedge \mathrm{d} y\right]\right\},
\end{aligned}
$$

with $\beta$ and $\omega$ given in (4.3b) and (4.3d). The term in the last line is d-trivial and can be gauged away. Notice that the perturbation (4.18a)-(4.18b) can be embedded, at linear order in $c_{l}$, in the ansatz (E.51), by choosing

$$
\begin{aligned}
& Z_{4}=\frac{c_{l}}{2} e^{-2 i l \phi} \frac{\sin ^{2 l} \theta}{\left(r^{2}+a^{2}\right)^{l}\left(r^{2}+a^{2} \cos ^{2} \theta\right)}, \quad a_{4}=0, \\
& \delta_{2}=-\frac{c_{l}}{2} e^{-2 i l \phi} \frac{\sin ^{2 l} \theta}{\left(r^{2}+a^{2}\right)^{l}}\left[\frac{r^{2}+a^{2}}{r^{2}+a^{2} \cos ^{2} \theta} \cos ^{2} \theta \mathrm{d} \phi \wedge \mathrm{d} \psi+i \frac{\cos \theta}{\sin \theta} \mathrm{d} \theta \wedge \mathrm{d} \psi\right] .
\end{aligned}
$$

It is immediate to check that the $Z_{4}$ and $\delta_{2}$ above satisfy the only supergravity constraint (E.57a) that is non-trivial for this solution, i.e.

$$
*_{4} \mathrm{~d} Z_{4}=\mathrm{d} \delta_{2} .
$$

Since all two-charge solutions are known and are associated with a curve $g_{A}\left(v^{\prime}\right)(A=$ $1, \ldots, 8)$ in $\mathbb{R}^{4} \times T^{4}$, the solution (4.19a)-(4.19b) is defined by a particular $g_{A}\left(v^{\prime}\right)$. As mentioned before, this curve represents the profile of the string in the duality frame in which the charges are fundamental string and momentum: the solutions corresponding to curves in $\mathbb{R}^{4}$ are the Lunin-Mathur geometries [53,54], while general curves where considered in $[41,58]$. Choosing a generic profile in $\mathbb{R}^{4} \times T^{4}$ for the vibrating string breaks the rotation invariance on $T^{4}$; however, when going from the F1-P to the D1-D5 duality frame, one of the directions of $T^{4}$, that we take to be the direction $A=5$, is singled out. In particular a profile $g_{A}\left(v^{\prime}\right)$ that has only component 5 in $T^{4}$ will be a scalar in the D1-D5 frame, while a profile in the other $T^{4}$ direction will correspond to a three-form. Then, D1-D5 geometries whose profile has components only along the directions $A=1, \ldots, 5$ are $T^{4}$ isotropic and have, generically, all type IIB fields excited. The solution dual to the state $|\Psi\rangle_{R}$ is exactly of this form, and indeed one can see that it coincides with the D1-D5 geometry associated with the curve

$\overline{17}$ To match the conventions of [42] with ours, one also needs to reverse the orientation: $*_{6} \rightarrow-*_{6}$, where, in our conventions, $\epsilon_{t y 1234}=+1$. 


$$
g_{1}\left(v^{\prime}\right)=a \cos \frac{2 \pi v^{\prime}}{L}, \quad g_{2}\left(v^{\prime}\right)=a \cos \frac{2 \pi v^{\prime}}{L}, \quad g_{5}\left(v^{\prime}\right)=-i b e^{-\frac{4 \pi i l v^{\prime}}{L}},
$$

at first order in $b$ and with all remaining components of $g$ set to zero. The relation between the parameters $b$ and $c_{l}$ is

$$
\frac{c_{l}}{2}=-b a R \text {. }
$$

We can embed in our ansatz also the solution in Eqs. (3.21)-(3.30) of [42] corresponding to the three-charge CFT state $J_{-1}^{-}|\Psi\rangle_{R}$. As discussed in the previous section this configuration is generated by combining the change of coordinates corresponding to the CFT spectral flow and $\mathbb{R}^{4}$ rotations. The solution carrying one unit of momentum is described by the following geometric data ${ }^{18}$

$$
\begin{aligned}
Z_{4}= & -c_{l} l e^{-i \sqrt{2} \frac{v}{R}} e^{-2 i l \phi+i(\phi+\psi)} \frac{\sin ^{2 l-1} \theta \cos \theta}{\left(r^{2}+a^{2}\right)^{l}\left(r^{2}+a^{2} \cos ^{2} \theta\right)} \\
\delta_{2}= & -c_{l} l e^{-i \sqrt{2} \frac{v}{R}} e^{-2 i l \phi+i(\phi+\psi)} \frac{r \sin ^{2 l-1} \theta}{\left(r^{2}+a^{2}\right)^{l}}\left[\sin \theta\left(\frac{\mathrm{d} r \wedge \mathrm{d} \theta}{r^{2}+a^{2}}+\frac{r \sin \theta \cos \theta}{r^{2}+a^{2} \cos ^{2} \theta} \mathrm{d} \phi \wedge \mathrm{d} \psi\right)\right. \\
& \left.-i\left(\frac{\cos \theta}{r^{2}+a^{2}} \mathrm{~d} r \wedge \mathrm{d} \psi+\frac{\sin \theta}{r} \mathrm{~d} \theta \wedge \mathrm{d} \phi\right)\right] .
\end{aligned}
$$

The other data describing the geometry remain, of course, unchanged at linear order and the only non-trivial supergravity equations are (E.57)

$$
\dot{\delta}_{2}=*_{4} \dot{\delta}_{2}, \quad *_{4} \mathcal{D} Z_{4}=\mathcal{D} \delta_{2},
$$

which are easily verified.

The advantage of rewriting the "near-horizon" MSS solution in the form of our ansatz is that the extension to the asymptotically flat region is now immediate: it is enough to re-add the " 1 " to the functions $Z_{1}$ and $Z_{2}$. As the equations for $Z_{4}$ and $\delta_{2}$ do not involve $Z_{1}$ an $Z_{2}$ (when, as in our case, $\beta$ is $v$-independent), it is evident that sending $Z_{1,2} \rightarrow Z_{1,2}+1$ does not change $Z_{4}$ and $\delta_{2}$. Moreover, inspection of the other supergravity constraints immediately shows that all the other geometric data are unmodified at first order in the perturbation parameter $c_{l}$. In conclusion, the geometry given by the data in (4.3a)-(4.3d) and in (4.24a)-(4.24b) solves the supergravity equations at first order in $c_{l}$ and interpolates between flat space and the AdS region for any value of $\epsilon=\frac{a}{\sqrt{Q}}$. Thus it represents a "perturbative" microstate of the three-charge black hole with one unit of momentum.

\subsection{The non-linear completion}

If one wants to describe microstates that carry a macroscopic amount of momentum charge (rather than just one quantum) one needs to take into account higher order contributions in the perturbation parameter $c_{l}$. The solution of the previous subsection fails to solve the supergravity equations at all orders in $c_{l}$ : terms of order $c_{l}^{2}$ appear on the r.h.s. of the equations involving $\mathcal{F}$

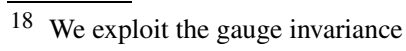

$$
a_{4} \rightarrow a_{4}-\dot{\lambda}^{(1)}, \quad \delta_{2} \rightarrow \delta_{2}+\mathcal{D} \lambda^{(1)},
$$

with $\lambda^{(1)}$ a 1-form, to set $a_{4}=0$. 
and $\omega$ (E.58) and thus these metric coefficients will necessarily be modified at that order. Note that the appearance of a non-vanishing $\mathcal{F}$ is expected for geometries carrying a finite amount of momentum charge.

One can construct a fully non-linear three-charge solution by starting from an exact twocharge geometry and applying a sequence of transformations similar to the ones described above. We will give here a sketch of the construction, specifying for simplicity to the case $l=\frac{1}{2}$.

Let us consider the non-linear extension of the two-charge microstate described by the curve in (4.21). First of all, when working at the non-linear level one has to use real expressions (as the trick of taking the real part of the final solution does not apply when the equations are non-linear). There are of course many possible curves that reduce to the real part of (4.21) at linear order in $b$. We will make here the following choice

$$
g_{1}\left(v^{\prime}\right)=a \cos \left(\frac{2 \pi v^{\prime}}{L}\right), \quad g_{2}\left(v^{\prime}\right)=a \sin \left(\frac{2 \pi v^{\prime}}{L}\right), \quad g_{5}\left(v^{\prime}\right)=-b \sin \left(\frac{2 \pi v^{\prime}}{L}\right),
$$

while all other components of the profile are trivial. The exact two-charge geometry corresponding to this profile can be derived thanks to the results of [41] and is given, in our notations, by $^{19}$

$$
\begin{aligned}
& \mathrm{d} s_{4}^{2}=\left(r^{2}+a^{2} \cos ^{2} \theta\right)\left(\frac{\mathrm{d} r^{2}}{r^{2}+a^{2}}+\mathrm{d} \theta^{2}\right)+\left(r^{2}+a^{2}\right) \sin ^{2} \theta \mathrm{d} \phi^{2}+r^{2} \cos ^{2} \theta \mathrm{d} \psi^{2}, \\
& \beta=\frac{R a^{2}}{\sqrt{2}\left(r^{2}+a^{2} \cos ^{2} \theta\right)}\left(\sin ^{2} \theta \mathrm{d} \phi-\cos ^{2} \theta \mathrm{d} \psi\right), \\
& Z_{1}=1+\frac{R^{2}}{Q_{5}} \frac{a^{2}+\frac{b^{2}}{2}}{r^{2}+a^{2} \cos ^{2} \theta}+\frac{R^{2} a^{2} b^{2}}{2 Q_{5}} \frac{\cos 2 \phi \sin ^{2} \theta}{\left(r^{2}+a^{2} \cos ^{2} \theta\right)\left(r^{2}+a^{2}\right)}, \\
& Z_{2}=1+\frac{Q_{5}}{r^{2}+a^{2} \cos ^{2} \theta}, \quad a_{1}=0, \\
& Z_{4}=R a b \frac{\cos \phi \sin \theta}{\sqrt{r^{2}+a^{2}}\left(r^{2}+a^{2} \cos ^{2} \theta\right)}, \quad a_{4}=0, \\
& \delta_{2}=\frac{-R a b \sin \theta}{\sqrt{r^{2}+a^{2}}}\left[\frac{r^{2}+a^{2}}{r^{2}+a^{2} \cos ^{2} \theta} \cos ^{2} \theta \cos \phi \mathrm{d} \phi \wedge \mathrm{d} \psi+\sin \phi \frac{\cos \theta}{\sin \theta} \mathrm{d} \theta \wedge \mathrm{d} \psi\right] \text {, } \\
& \omega=\frac{R a^{2}}{\sqrt{2}\left(r^{2}+a^{2} \cos ^{2} \theta\right)}\left(\sin ^{2} \theta \mathrm{d} \phi+\cos ^{2} \theta \mathrm{d} \psi\right), \\
& \mathcal{F}=0 \text {. }
\end{aligned}
$$

The relation (4.2) between the $y$ radius $R$ and the charges is now modified as

$$
R=\sqrt{\frac{Q_{1} Q_{5}}{a^{2}+\frac{b^{2}}{2}}}
$$

19 From now on we work with arbitrary D1 and D5 charges; of course, in order to find the results of the previous two subsections one needs to use explicitly the constraint $Q_{1}=Q_{5}=Q$. 
One can now take the "near-horizon" limit, ${ }^{20}$ which amounts to replacing $Z_{1,2} \rightarrow Z_{1,2}-1$, do a spectral flow to the NSNS sector, make a finite rotation in $\mathbb{R}^{4}$, and finally spectral flow back to the RR sector. One generates in this way a solution describing a three-charge microstate in the "near-horizon" region. The solution is

$$
\begin{aligned}
& \mathrm{d} s_{4}^{2}=\left(r^{2}+a^{2} \cos ^{2} \theta\right)\left(\frac{\mathrm{d} r^{2}}{r^{2}+a^{2}}+\mathrm{d} \theta^{2}\right)+\left(r^{2}+a^{2}\right) \sin ^{2} \theta \mathrm{d} \phi^{2}+r^{2} \cos ^{2} \theta \mathrm{d} \psi^{2}, \\
& \beta=\frac{R a^{2}}{\sqrt{2}\left(r^{2}+a^{2} \cos ^{2} \theta\right)}\left(\sin ^{2} \theta \mathrm{d} \phi-\cos ^{2} \theta \mathrm{d} \psi\right), \\
& Z_{1}=\frac{R^{2}}{Q_{5}} \frac{a^{2}+\frac{b^{2}}{2}}{r^{2}+a^{2} \cos ^{2} \theta}+\frac{R^{2} a^{2} b^{2}}{2 Q_{5}} \cos 2 \hat{v} \frac{\cos ^{2} \theta}{\left(r^{2}+a^{2} \cos ^{2} \theta\right)\left(r^{2}+a^{2}\right)}, \\
& Z_{2}=\frac{Q_{5}}{r^{2}+a^{2} \cos ^{2} \theta}, \quad a_{1}=0, \\
& Z_{4}=R a b \cos \hat{v} \frac{\cos \theta}{\sqrt{r^{2}+a^{2}}\left(r^{2}+a^{2} \cos ^{2} \theta\right)}, \quad a_{4}=0, \\
& \delta_{2}=R a b \frac{r}{\sqrt{r^{2}+a^{2}}}\left[\cos \hat{v} \sin \theta\left(\frac{\mathrm{d} r \wedge \mathrm{d} \theta}{r^{2}+a^{2}}+\frac{r \sin \theta \cos \theta}{r^{2}+a^{2} \cos ^{2} \theta} \mathrm{d} \phi \wedge \mathrm{d} \psi\right)\right. \\
& \left.-\sin \hat{v}\left(\frac{\cos \theta}{r^{2}+a^{2}} \mathrm{~d} r \wedge \mathrm{d} \psi+\frac{\sin \theta}{r} \mathrm{~d} \theta \wedge \mathrm{d} \phi\right)\right] \text {, } \\
& \omega=\frac{R a^{2}}{\sqrt{2}\left(r^{2}+a^{2} \cos ^{2} \theta\right)}\left(\sin ^{2} \theta \mathrm{d} \phi+\cos ^{2} \theta \mathrm{d} \psi\right) \\
& +\frac{R b^{2}}{\sqrt{2}} \frac{\left(r^{2}+a^{2}\right) \sin ^{2} \theta \mathrm{d} \phi+r^{2} \cos ^{2} \theta \mathrm{d} \psi}{\left(r^{2}+a^{2}\right)\left(r^{2}+a^{2} \cos ^{2} \theta\right)}, \\
& \mathcal{F}=-\frac{b^{2}}{r^{2}+a^{2}}
\end{aligned}
$$

with

$$
\hat{v}=\frac{\sqrt{2} v}{R}-\psi .
$$

Note that the sequence of spectral flows and rotations in general mixes the coordinates $u$ and $v$ with the $\mathbb{R}^{4}$ coordinates; thus, one would have expected that the $4 \mathrm{D}$ metric $\mathrm{d} s_{4}^{2}$ and the 1 -form $\beta$ would have been modified by the series of change of coordinates performed. However, this has not happened for the particular transformations that generate the geometry corresponding to the state $J_{-1}^{-}|\Psi\rangle_{R}$ : at the end $\mathrm{d} s_{4}^{2}$ is still flat and $\beta$ is still $v$-independent. Note also that $a_{1}$ is still vanishing and $Z_{2}$ is still $v$-independent, which implies that $\Theta_{1}=0$. One has instead a non-vanishing $\Theta_{2}$, that can be computed to be

$$
\begin{aligned}
\Theta_{2}= & -\frac{\sqrt{2} R a^{2} b^{2}}{Q_{5}} \frac{r \cos \theta}{r^{2}+a^{2}}\left[\sin 2 \hat{v} \sin \theta\left(\frac{\mathrm{d} r \wedge \mathrm{d} \theta}{r^{2}+a^{2}}+\frac{r \sin \theta \cos \theta}{r^{2}+a^{2} \cos ^{2} \theta} \mathrm{d} \phi \wedge \mathrm{d} \psi\right)\right. \\
& \left.+\cos 2 \hat{v}\left(\frac{\cos \theta}{r^{2}+a^{2}} \mathrm{~d} r \wedge \mathrm{d} \psi+\frac{\sin \theta}{r} \mathrm{~d} \theta \wedge \mathrm{d} \phi\right)\right] .
\end{aligned}
$$

$\overline{20}$ In the "near-horizon" limit both $a^{2}$ and $b^{2}$ are much smaller than $\sqrt{Q_{1} Q_{5}}$. 
Being generated from a regular solution via a globally defined sequence of coordinate transformations, the geometry $(4.29 \mathrm{a})-(4.29 \mathrm{~h})$ is guaranteed to solve the supergravity constraints and to be regular.

The final task is the extension of the above "near-horizon" solution to one that has flat asymptotics. This task is complicated in this case by two factors: first, the replacement $Z_{1,2} \rightarrow Z_{1,2}+1$ generates on the r.h.s. of Eqs. (E.38) and (E.58b) terms proportional to $\Theta_{2}$ and to the $v$-derivatives of $Z_{1}$ and forces $\omega$ and/or $\mathcal{F}$ to be corrected in order to preserve the supergravity constraints. Second, the necessary corrections to $\omega$ spoil the regularity of the geometry and have to be further compensated by corrections of order $\frac{a^{2}}{Q_{5}}$. We will not attempt to explain here a systematic technique to address these problems. The recent results of [59] have provided a general method to produce $v$-dependent solutions of the supergravity equations precisely in the situation that is relevant for our problem, i.e. when the 4D metric $\mathrm{d}_{4}^{2}$ and $\beta$ are $v$-independent. The construction of [59] applies to the restricted ansatz in which $Z_{4}=a_{4}=\delta_{2}=0$, but its extension to our more general set up is straightforward. We have checked that the solution (4.29a)-(4.29h) fits into the scheme of [59], and we have used this observation to generate the corresponding asymptotically flat geometry. We leave the details, as well as further applications, to a forthcoming work. We quote here, for completeness, the final solution:

$$
\begin{aligned}
& \mathrm{d} s_{4}^{2}=\left(r^{2}+a^{2} \cos ^{2} \theta\right)\left(\frac{\mathrm{d} r^{2}}{r^{2}+a^{2}}+\mathrm{d} \theta^{2}\right)+\left(r^{2}+a^{2}\right) \sin ^{2} \theta \mathrm{d} \phi^{2}+r^{2} \cos ^{2} \theta \mathrm{d} \psi^{2}, \\
& \beta=\frac{R a^{2}}{\sqrt{2}\left(r^{2}+a^{2} \cos ^{2} \theta\right)}\left(\sin ^{2} \theta \mathrm{d} \phi-\cos ^{2} \theta \mathrm{d} \psi\right), \\
& Z_{1}=1+\frac{R^{2}}{Q_{5}} \frac{a^{2}+\frac{b^{2}}{2}}{r^{2}+a^{2} \cos ^{2} \theta}+\frac{R^{2} a^{2} b^{2}}{2\left(Q_{5}+a^{2}\right)} \frac{\cos 2 \hat{v} \cos ^{2} \theta}{\left(r^{2}+a^{2} \cos ^{2} \theta\right)\left(r^{2}+a^{2}\right)}, \\
& Z_{2}=1+\frac{Q_{5}}{r^{2}+a^{2} \cos ^{2} \theta}, \quad a_{1}=0, \\
& Z_{4}=R a b \cos \hat{v} \frac{\cos \theta}{\sqrt{r^{2}+a^{2}}\left(r^{2}+a^{2} \cos ^{2} \theta\right)}, \quad a_{4}=0, \\
& \delta_{2}=R a b \frac{r}{\sqrt{r^{2}+a^{2}}}\left[\cos \hat{v} \sin \theta\left(\frac{\mathrm{d} r \wedge \mathrm{d} \theta}{r^{2}+a^{2}}+\frac{r \sin \theta \cos \theta}{r^{2}+a^{2} \cos ^{2} \theta} \mathrm{d} \phi \wedge \mathrm{d} \psi\right)\right. \\
& \left.-\sin \hat{v}\left(\frac{\cos \theta}{r^{2}+a^{2}} \mathrm{~d} r \wedge \mathrm{d} \psi+\frac{\sin \theta}{r} \mathrm{~d} \theta \wedge \mathrm{d} \phi\right)\right], \\
& \omega=\frac{R a^{2}}{\sqrt{2}\left(r^{2}+a^{2} \cos ^{2} \theta\right)}\left(\sin ^{2} \theta \mathrm{d} \phi+\cos ^{2} \theta \mathrm{d} \psi\right) \\
& +\frac{R b^{2}}{\sqrt{2}} \frac{\left(r^{2}+a^{2}\right) \sin ^{2} \theta \mathrm{d} \phi+r^{2} \cos ^{2} \theta \mathrm{d} \psi}{\left(r^{2}+a^{2}\right)\left(r^{2}+a^{2} \cos ^{2} \theta\right)} \\
& -\frac{R a^{2} b^{2}}{2 \sqrt{2}\left(Q_{5}+a^{2}\right)}\left[\cos 2 \hat{v} \frac{a^{2} \sin ^{2} \theta \mathrm{d} \phi-r^{2} \mathrm{~d} \psi}{\left(r^{2}+a^{2}\right)\left(r^{2}+a^{2} \cos ^{2} \theta\right)} \cos ^{2} \theta\right. \\
& \left.+\sin 2 \hat{v} \frac{r \cos \theta \mathrm{d} r-\left(r^{2}+a^{2}\right) \sin \theta \mathrm{d} \theta}{\left(r^{2}+a^{2}\right)^{2}} \cos \theta\right] \text {, } \\
& \mathcal{F}=-\frac{b^{2}}{r^{2}+a^{2}} .
\end{aligned}
$$


This geometry solves the non-linear supergravity equations, is regular and horizon-less, and at large distances reduces to the geometry of the black hole with D1, D5 and P charges.

\section{Acknowledgements}

We thank I. Bena, G. Dall'Agata, G. Gibbons, S. Mathur, M. Shigemori, A. Tomasiello, D. Turton and N. Warner for several enlightening discussions. We especially thank I. Bena for useful comments on the draft. S.G. and L.M. have been partially supported by MIUR-PRIN contract 2009-KHZKRX, by the Padova University Project CPDA119349 and by INFN. R.R. has been partially supported by STFC Standard Grant ST/J000469/1 'String Theory, Gauge Theory and Duality'. S.G., M.P. and R.R. have been partially supported by the CNRS grant PICS "Aspects of String Theory with Fluxes". M.P. wishes to thank the Theory Group of Imperial College, London, for hospitality during the completion of this work. R.R. wishes to thank Université Pierre et Marie Curie (Paris), Università di Torino and Collège de France for hospitality, and the 'Institut Lagrange de Paris' for support during the completion of this work.

\section{Appendix A. Conventions}

In this paper we will consider ten-dimensional Minkowski space we use the signature $(-,+, \ldots,+)$, as well as eight- and four-dimensional Euclidean space. We distinguish local flat indices by underlying them.

In a $D$-dimensional space, take an oriented vielbein $e^{\underline{m}}$, with $\underline{m}=0, \ldots, D-1$ if the space is Minkowskian and $\underline{m}=1, \ldots, D$ if it is Euclidean. Then the Hodge- $*$ of a $k$-form $A_{k}$ is defined as follows:

$$
* A_{k} \equiv \frac{1}{k !(D-k) !} \epsilon_{\underline{m}_{1} \ldots \underline{m}_{D-k} \underline{m}_{D-k+1} \ldots \underline{m}_{D}} A^{\underline{m}_{D-k+1} \cdots \underline{m}_{D}} e^{\underline{m}_{1}} \wedge \cdots \wedge e^{\underline{m}_{D-k}},
$$

where $\epsilon_{\underline{m}_{1} \ldots \underline{m}_{D}}$ is the totally antisymmetric symbol, such that $\epsilon_{\underline{0} \ldots \underline{D-1}}=1$ and $\epsilon_{\underline{1} \ldots \underline{D}}=1$ in Minkowskian and Euclidean spaces respectively.

We often use the operator $\lambda$ which acts on a $k$-form $A_{k}$ as follows:

$$
\lambda\left(A_{k}\right)=(-)^{k(k-1) / 2} A_{k},
$$

that is, $\lambda$ exchanges the order of the indices of the form it is action on.

We define the contraction of a $k$-form $A_{k}$ by a vector $X=X^{m} \partial_{m}$ by

$$
\iota_{X} A_{k} \equiv \frac{1}{(k-1) !} X^{m} A_{m n_{1} \ldots n_{k-1}} \mathrm{~d} x^{n_{1}} \wedge \cdots \wedge \mathrm{d} x^{n_{k-1}},
$$

and we use the shorthand notation $\iota_{m} \equiv \iota_{\partial_{m}}$.

We also make use of the full contraction of two $k$-forms, $A_{k}$ and $B_{k}$,

$$
A_{k} \cdot B_{k} \equiv \frac{1}{p !} A_{M_{1} \ldots M_{p}} B^{M_{1} \ldots M_{p}},
$$

which is generalised to the contraction of polyforms $A=\sum_{k} A_{k}$ and $B=\sum_{k} B_{k}$ as

$$
A \cdot B \equiv \sum_{k} A_{k} \cdot B_{k}
$$

When acting on spinors, a $k$-form is implicitly taken to be contracted by gamma matrices 


$$
A_{k} \equiv \frac{1}{k !} A_{m_{1} \ldots m_{k}} \gamma^{m_{1} \ldots m_{k}}
$$

where, as usual, $\gamma^{m_{1} \ldots m_{k}} \equiv \gamma^{\left[m_{1}\right.} \ldots \gamma^{\left.m_{k}\right]}$.

We use spinor in 10 Minkowskian and 8 and 4 Euclidean dimensions. The corresponding chirality operators are

$$
\Gamma_{(10)}=\Gamma^{0 . . .9}, \quad \gamma_{(8)}=\gamma^{1 \ldots 8}, \quad \gamma_{(4)}=\gamma^{1 \ldots 4} .
$$

When we consider the split from 10 to $2+8$ dimensions we use the following gamma matrices decomposition

$$
\Gamma^{\underline{0}}=\mathrm{i} \sigma_{2} \otimes \gamma_{(8)}, \quad \Gamma^{\underline{1}}=\sigma_{1} \otimes \gamma_{(8)}, \quad \Gamma^{\underline{a}}=\mathbb{1} \otimes \gamma^{\underline{a}} .
$$

We can also split the ten-dimensional charge conjugation matrix

$$
C_{(10)}=\mathrm{i} \sigma_{2} \otimes C_{(8)} \gamma_{(8)},
$$

where $C_{(8)}$ is such that $C_{(8)} \gamma_{\underline{a}} C_{(8)}^{-1}=\gamma_{\underline{a}}^{T}$. The Majorana condition on a ten-dimensional spinor $\epsilon$ imposes that

$$
\bar{\epsilon} \equiv \epsilon^{\dagger} \Gamma^{0}=\epsilon^{T} C_{(10)},
$$

and on an eight-dimensional spinor $\eta$ imposes

$$
\eta^{\dagger}=\eta^{T} C_{(8)} \text {. }
$$

By choosing $C_{(8)}=\mathbb{1}$ and then $C_{(10)}=\Gamma^{0}$ one obtains the real representation.

We also consider the split of the Euclidean 8 dimensions into $4+4$ dimensions as in (3.4), under which

$$
C_{(8)}=C_{(4)} \otimes \hat{C}_{(4)}
$$

where $C_{(4)}$ (and analogously $\hat{C}_{(4)}$ ) now satisfies the identities $C_{(4)} \gamma_{i} C_{(4)}^{-1}=-\gamma_{i}^{T}$. For instance, we could take the following explicit representation for four-dimensional gamma matrices

$$
\gamma^{1}=\sigma_{1} \otimes \mathbb{1}, \quad \gamma^{2}=\sigma_{2} \otimes \mathbb{1}, \quad \gamma^{\underline{3}}=\sigma_{3} \otimes \sigma_{2}, \quad \gamma^{4}=\sigma_{3} \otimes \sigma_{1}
$$

and then $\gamma_{(4)}=\sigma_{3} \otimes \sigma_{3}$. In this basis we can choose $C_{(4)}=\sigma_{2} \otimes \sigma_{1}$.

In this paper we use the democratic formulation [60] of type II supergravities in the conventions spelt out in detail in Appendix A of [61], up to a sign flip $H \rightarrow-H$. Let us just recall some informations relevant for the analysis of supersymmetry.

For the Ramond-Ramond fields we consider the full sum of field strengths

$$
F=\sum_{k} F_{k},
$$

with $k$ even (from 0 to 10 ) in IIA and odd (from 1 to 9) in IIB. The redundant degrees of freedom in $F$ are eliminated by the self-duality constraint

$$
F=* \lambda(F),
$$

where $\lambda$ is given in (A.2).

The fermionic content of type II supergravity consists of a doublet of gravitino's and dilatino's

$$
\psi_{M}=\left(\psi_{M}^{1}, \psi_{M}^{2}\right), \quad \lambda=\left(\lambda^{1}, \lambda^{2}\right)
$$


The components of the doublet have different chirality in type IIA and the same chirality in type IIB. In both theories we fix the chirality to be

$$
\Gamma_{(10)} \psi_{M}^{1}=\psi_{M}^{1}, \quad \Gamma_{(10)} \lambda^{1}=-\lambda^{1} .
$$

The type II supersymmetry transformations are parameterised by a doublet of Majorana-Weyl spinors $\epsilon=\left(\epsilon_{1}, \epsilon_{2}\right)$, of opposite chirality in IIA and same chirality in IIB

$$
\Gamma_{(10)} \epsilon_{1}=\epsilon_{1} \quad(\mathrm{IIA}), \quad \Gamma_{(10)} \epsilon_{2}=\mp \epsilon_{2} \quad \text { (IIB). }
$$

In our conventions, the type II supersymmetry transformations of [60] can be written as follows:

$$
\begin{aligned}
& \delta \psi_{M}^{1}=\left(\nabla_{M}-\frac{1}{4} \iota_{M} H\right) \epsilon_{1}+\frac{1}{16} e^{\phi} F \Gamma_{M} \Gamma_{(10)} \epsilon_{2}, \\
& \delta \psi_{M}^{2}=\left(\nabla_{M}+\frac{1}{4} \iota_{M} H\right) \epsilon_{2}-\frac{1}{16} e^{\phi} \lambda(F) \Gamma_{M} \Gamma_{(10)} \epsilon_{1}, \\
& \delta \lambda^{1}=\left(\mathrm{d} \phi-\frac{1}{2} H\right) \epsilon_{1}+\frac{1}{16} e^{\phi} \Gamma^{M} F \Gamma_{M} \Gamma_{(10)} \epsilon_{2}, \\
& \delta \lambda^{2}=\left(\mathrm{d} \phi+\frac{1}{2} H\right) \epsilon_{2}-\frac{1}{16} e^{\phi} \Gamma^{M} \lambda(F) \Gamma_{M} \Gamma_{(10)} \epsilon_{1},
\end{aligned}
$$

where fluxes are contracted on gamma matrices as in (A.6).

\section{Appendix B. Spin(7) structures}

Take an 8-dimensional space $X$ and a globally defined, nowhere vanishing Majorana-Weyl spinor $\eta$. The structure group oh the spin bundle is reduced from $\operatorname{Spin}(8)$ to $\operatorname{Spin}(7)$. We choose the charge conjugation matrix $C_{(8)}=\mathbb{1}$, so that all gamma matrices $\gamma_{a}$ are real, $\eta$ is real and satisfies $\gamma_{(8)} \eta=\eta$, with $\gamma_{(8)}=\gamma_{1 \ldots 8}$. Furthermore we normalise $\eta$ in such a way that

$$
\eta^{T} \eta=1 \text {. }
$$

An equivalent way of defining a reduced $\operatorname{Spin}(7)$ structure is via a four-form

$$
\Omega=\frac{1}{4 !} \eta^{T} \gamma_{a b c d} \eta \mathrm{d} y^{a} \wedge \mathrm{d} y^{b} \wedge \mathrm{d} y^{c} \wedge \mathrm{d} y^{d}
$$

If $\eta$ has positive chirality, then $\Omega$ is self-dual

$$
* \Omega=\Omega,
$$

while if $\gamma_{(8)} \eta=-\eta$ then $* \Omega=-\Omega$.

The different tensors can be decomposed in different representations of Spin(7). A vector transforms in the representation 8 of $\operatorname{Spin}(7)$. On the other hand, a tensor with two antisymmetric indices decomposes as follows in irreducible representations:

$$
\alpha_{[a b]}=\mathbf{7} \oplus \mathbf{2 1}
$$

We can use $\Omega$ to construct the corresponding projectors. In particular

$$
\left(P_{7}\right)_{a b}^{c d}=\frac{1}{4}\left(\delta_{[a}^{[c} \delta_{b]}^{d]}-\frac{1}{2} \Omega_{a b}{ }^{c d}\right)
$$

is the projector on the 7 of $\operatorname{Spin}(7)$. 
It is also useful to observe that, if $\chi$ is any four-form, then one can construct a two-form transforming in the 7 of $\operatorname{Spin}(7)$

$$
T^{7} \equiv \iota_{[a} \Omega \cdot \iota_{b]} \chi \mathrm{d} y^{a} \wedge \mathrm{d} y^{b} \equiv \frac{1}{3 !}\left(\Omega_{[a}^{c d e} \chi_{b] c d e}\right) \wedge \mathrm{d} y^{a} \wedge \mathrm{d} y^{b} .
$$

\section{B.1. Decomposition of spinors}

We can decompose an arbitrary spinor $\xi$ on $X$ by using as basis $\eta, \gamma_{a} \eta, \gamma_{a b} \eta$. In particular, if $\eta$ has positive chirality, we can decompose a positive/negative chirality spinor $\xi_{+/-}$as

$$
\begin{aligned}
& \zeta_{+}=\chi^{\mathbf{1}} \eta+\frac{1}{2 !} \chi_{a b}^{7} \gamma^{a b} \eta, \\
& \zeta_{-}=\chi_{a}^{\mathbf{8}} \gamma^{a} \eta,
\end{aligned}
$$

where $\chi^{\mathbf{1}}$ is a singlet of $\operatorname{Spin}(7), \chi^{\mathbf{8}}$ is a vector and the two-form $\chi_{a b}^{\mathbf{7}}$ transforms as 7 of $\operatorname{Spin}(7)$. This is due to the following projector conditions:

$$
\left(P_{7}\right)_{a b}^{c d} \gamma_{c d} \eta=\gamma_{c d} \eta \text {. }
$$

Hence $\zeta_{+} \in \mathbf{1} \oplus \mathbf{7}$ and $\zeta_{-} \in \mathbf{8}$. Notice also that $\left(\eta, \gamma_{a b} \eta\right)$ and $\left(\gamma^{a} \eta\right)$ form a basis for the positive and negative chirality spinors, respectively. If $\gamma_{(8)} \eta=-\eta$, we have a similar decomposition with chiral and antichiral spinors exchanged.

The spinorial basis $\left(\eta, \gamma_{a} \eta, \gamma_{a b} \eta\right)$ obeys the following orthogonality conditions

$$
\begin{aligned}
& \eta^{T} \gamma_{a} \gamma^{b} \eta=\delta_{a}^{b}, \\
& \eta^{T} \gamma_{a b} \gamma^{c d} \eta=-8\left(P_{7}\right)_{a b}{ }^{c d},
\end{aligned}
$$

which can be used to invert the decomposition (B.7)

$$
\chi^{1}=\eta^{T} \zeta_{+}, \quad \chi_{a b}^{7}=-\frac{1}{4} \eta^{T} \gamma_{a b} \zeta_{+}, \quad \chi_{a}^{8}=\eta^{T} \gamma_{a} \zeta_{-} .
$$

Another useful identity is the following. Let us state it for a non-normalised spinor $\eta$, with $\eta^{T} \eta \neq 1$. If we define a (non-normalised) $\Omega_{a b c d}=\eta^{T} \gamma_{a b c d} \eta$, then

$$
\eta^{T} \gamma_{a b} \dot{\eta}-\frac{1}{2} \eta^{T} \gamma_{a b} m_{\mathrm{A}} \eta=-\frac{1}{8 \eta^{T} \eta} \iota_{[a} \Omega \cdot \frac{\mathrm{d}}{\mathrm{d} v}\left(\iota_{b} \Omega\right) .
$$

Notice that all terms in this equations transform as two-forms in 7, cf. (B.6) and (B.8).

\section{Appendix C. Integrability for null Killing vectors}

In this section we discuss again the relation between supersymmetry and equations of motion for the case of null Killing vector, which is relevant for this paper. In the derivation we do not explicitly include branes as localised sources and we only consider the closed string sector.

Let us first define

$$
\begin{aligned}
& \mathcal{E}_{M N}=R_{M N}+2 \nabla_{M} \nabla_{N} \phi-\frac{1}{2} H_{M} \cdot H_{N}-\frac{1}{4} e^{2 \phi} F_{M} \cdot F_{N}, \\
& \mathcal{H}_{M N}=e^{2 \phi} *_{10}\left[\mathrm{~d}\left(e^{-2 \phi} *_{10} H\right)+\frac{1}{2}\left(*_{10} F \wedge F\right)_{8}\right], \\
& \mathcal{O}=2 R-H^{2}+8\left[\nabla^{2} \phi-(\mathrm{d} \phi)^{2}\right],
\end{aligned}
$$


where $F$ is the sum of all RR filed-strengths as in (A.14). Using this notation, the string-frame trace-reversed Einstein equations, the $B$-field equations and the dilation's equation are simply

$$
\mathcal{E}_{M N}=0, \quad \mathcal{H}_{M N}=0, \quad \mathcal{O}=0 .
$$

On the other hand,

$$
\mathrm{d} H=0, \quad \mathrm{~d}_{H} F=0
$$

give the Bianchi identity for the $B$ field and the Bianchi identities and equations of motion for the RR-fields.

The starting point of our discussion is provided by Eqs. (11.4) in [61]. They immediately imply that, if the type II background is supersymmetric, the following equations are satisfied

$$
\begin{aligned}
& \mathcal{E}_{M N} \Gamma^{N} \epsilon_{1}+\frac{1}{2}\left[\mathcal{H}_{M N} \Gamma^{N}+\iota_{M}(\mathrm{~d} H)\right] \epsilon_{1}+\frac{1}{4}\left(\mathrm{~d}_{H} F\right) \Gamma_{M} \Gamma_{(10)} \epsilon_{2}=0, \\
& \mathcal{E}_{M N} \Gamma^{N} \epsilon_{2}-\frac{1}{2}\left[\mathcal{H}_{M N} \Gamma^{N}+\iota_{M}(\mathrm{~d} H)\right] \epsilon_{2}-\frac{1}{4} \Gamma_{(10)} \lambda\left(\mathrm{d}_{H} F\right) \Gamma_{M} \epsilon_{1}=0, \\
& \mathcal{O} \epsilon_{1}+2(\mathrm{~d} H) \epsilon_{1}-\left(\mathrm{d}_{H} F\right) \epsilon_{2}=0, \\
& \mathcal{O} \epsilon_{2}-2(\mathrm{~d} H) \epsilon_{2}-\lambda\left(\mathrm{d}_{H} F\right) \epsilon_{1}=0 .
\end{aligned}
$$

If we then impose the Bianchi identities (C.3), it is easy to see from the last two equations in (C.4) that the dilaton equation, $\mathcal{O}=0$, is automatically fulfilled. On the other hand, the first two equations of (C.4) reduce to

$$
\begin{aligned}
& \left(\mathcal{E}_{M N}+\frac{1}{2} \mathcal{H}_{M N}\right) \Gamma^{N} \epsilon_{1}=0, \\
& \left(\mathcal{E}_{M N}-\frac{1}{2} \mathcal{H}_{M N}\right) \Gamma^{N} \epsilon_{2}=0 .
\end{aligned}
$$

These can be reduced to a set of bosonic conditions using the fact that $\Gamma^{\underline{u}} \epsilon_{1}$ and $\Gamma^{\underline{a}} \epsilon_{1}$, with $a=1, \ldots, 8$, form a set of linearly independent spinors ${ }^{21}$ (the same is true for $\epsilon_{2}$ )

$$
\begin{aligned}
\mathcal{E}_{\underline{M u}}+\frac{1}{2} \mathcal{H}_{\underline{M u}} & =0, & \mathcal{E}_{\underline{M a}}+\frac{1}{2} \mathcal{H}_{\underline{M a}} & =0, \\
\mathcal{E}_{\underline{M u}}-\frac{1}{2} \mathcal{H}_{\underline{M u}} & =0, & \mathcal{E}_{\underline{M a}}-\frac{1}{2} \mathcal{H}_{\underline{M a}} & =0,
\end{aligned}
$$

where again $\underline{a}=1, \ldots, 8$. Clearly, these are in turn equivalent to the set of equations

$$
\begin{array}{ll}
\mathcal{E}_{\underline{M u}}=0, & \mathcal{E}_{\underline{M a}}=0, \\
\mathcal{H}_{\underline{M u}}=0, & \mathcal{H}_{\underline{M a}}=0 .
\end{array}
$$

Since $\mathcal{H}_{M N}$ is antisymmetric, the second line of (C.7) is actually equivalent to the complete set of $B$-field equations of motion $\mathcal{H}_{M N}=0$. The first line provides almost all components of the Einstein equations $\mathcal{E}_{M N}=0$, with the exclusion of the $\underline{v v}$ component

$$
\mathcal{E}_{\underline{v v}}=0 .
$$

We then reach the conclusion that, if $K$ is null, imposing supersymmetry and the BI's (C.3) automatically implies all remaining equations of motion but (C.8), which then needs to be checked separately.

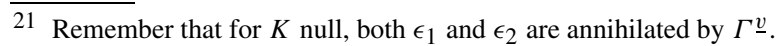




\section{Appendix D. The missing supersymmetry equations}

Eqs. (2.3) and (2.6) are not sufficient for guaranteeing the supersymmetry of a ten-dimensional background and must be supplemented by additional conditions [12]. The problem is that some information contained in the Killing spinor equations is projected out when going to the polyform equations. In fact, by looking at Appendix B of [12] one can easily identify which are the Killing spinor equations which are not captured by (2.3) and (2.6). The missing conditions are provided by Eqs. (B.39) and (B.40) therein and correspond exactly to the component of two gravitino equations along the null-directions $e_{+_{1}}$ and $e_{+_{2}}$.

In our case, where $K$ is null, we can identify $e_{+_{1}}$ and $e_{+_{2}}$ with $E_{\underline{v}}$ and then the missing supersymmetry conditions are given by the $\underline{v}$-component of the gravitino variations given in (A.19):

$$
\begin{aligned}
& \left(\nabla_{\underline{v}}-\frac{1}{4} \iota_{\underline{v}} H\right) \epsilon_{1}+\frac{1}{16} e^{\phi} F \Gamma_{\underline{v}} \Gamma_{(10)} \epsilon_{2}=0, \\
& \left(\nabla_{\underline{v}}+\frac{1}{4} \iota_{\underline{v}} H\right) \epsilon_{2}-\frac{1}{16} e^{\phi} \lambda(F) \Gamma_{\underline{v}} \epsilon_{1}=0 .
\end{aligned}
$$

Our aim is to express these equations in a more tractable form, different (but equivalent) to Eqs. (3.1d)-(3.1e) of [12].

We first split the NSNS and RR fluxes as in (2.16)

$$
\begin{aligned}
& H=h+E^{\underline{u}} \wedge h_{\underline{u}}+E^{\underline{v}} \wedge h_{\underline{v}}+E^{\underline{u}} \wedge E^{\underline{v}} \wedge h_{\underline{u v}}, \\
& F=f+E^{\underline{u}} \wedge f_{\underline{u}}+E^{\underline{v}} \wedge f_{\underline{v}}+E^{\underline{u}} \wedge E^{\underline{v}} \wedge f_{\underline{u v}} .
\end{aligned}
$$

Then we compute the covariant derivatives using the ansatz for the metric (2.13) and the spinors (2.22)

$$
\begin{aligned}
\nabla_{\underline{v}} \epsilon_{1} \equiv E_{\underline{v}}^{M} \nabla_{M} \epsilon_{1}= & \left(\begin{array}{l}
1 \\
0
\end{array}\right) \otimes\left[e^{-2 D} \dot{\eta}_{1}+\frac{1}{4}(\mathcal{D} \omega+W \mathcal{D} \beta) \eta_{1}-\frac{1}{2} e^{-2 D} m_{\mathrm{A}} \eta_{1}\right] \\
& -\left(\begin{array}{l}
0 \\
1
\end{array}\right) \otimes\left[\frac{\sqrt{2}}{4}\left(e^{-2 D} \mathcal{D} e^{2 D}-\dot{\beta}\right) \eta_{1}\right],
\end{aligned}
$$

where we have introduced the two-form

$$
m_{\mathrm{A}}=\frac{1}{2} \delta_{\underline{a c}} \dot{e}_{d}^{\underline{c}} e_{\underline{b}}^{d} e^{\underline{a}} \wedge e^{\underline{b}}
$$

Analogously

$$
\begin{aligned}
\nabla_{\underline{v}} \epsilon_{2}=E_{\underline{v}}^{M} \nabla_{M} \epsilon_{1}= & \left(\begin{array}{l}
1 \\
0
\end{array}\right) \otimes\left[e^{-2 D} \dot{\eta}_{2}+\frac{1}{4}(\mathcal{D} \omega+W \mathcal{D} \beta) \eta_{2}-\frac{1}{2} e^{-2 D} m_{\mathrm{A}} \eta_{2}\right] \\
& +\left(\begin{array}{l}
0 \\
1
\end{array}\right) \otimes\left[(-)^{|F|} \frac{\sqrt{2}}{4}\left(e^{-2 D} \mathcal{D} e^{2 D}-\dot{\beta}\right) \eta_{2}\right] .
\end{aligned}
$$

The terms in (D.1) containing the fluxes are easily simplified using (D.2) and the chirality properties of the spinors. Then, we can rewrite (D.1) as

$$
\begin{aligned}
& \left(e^{-2 D} \mathcal{D} e^{2 D}-\dot{\beta}\right) \eta_{1}-h_{\underline{u v}} \eta_{1}-\frac{1}{2} e^{\phi} f \eta_{2}=0, \\
& \left(e^{-2 D} \mathcal{D} e^{2 D}-\dot{\beta}\right) \eta_{2}+h_{\underline{u v}} \eta_{2}-\frac{1}{2}(-)^{|f|} e^{\phi} \lambda(f) \eta_{1}=0,
\end{aligned}
$$




$$
\begin{aligned}
& \dot{\eta}_{1}-\frac{1}{2} m_{\mathrm{A}} \eta_{1}+\frac{e^{2 D}}{4}(\mathcal{D} \omega+W \mathcal{D} \beta) \eta_{1}-\frac{e^{2 D}}{4} h_{\underline{v}} \eta_{1}+\frac{1}{8} e^{2 D+\phi} f_{\underline{v}} \eta_{2}=0, \\
& \dot{\eta}_{2}-\frac{1}{2} m_{\mathrm{A}} \eta_{2}+\frac{e^{2 D}}{4}(\mathcal{D} \omega+W \mathcal{D} \beta) \eta_{2}+\frac{e^{2 D}}{4} h_{\underline{v}} \eta_{2}-\frac{e^{2 D+\phi}}{8} \lambda\left(f_{\underline{v}}\right) \eta_{1}=0 .
\end{aligned}
$$

Each of the spinors $\eta_{1}$ and $\eta_{2}$ defines a $\operatorname{Spin}(7)$ structure. We can use them to expand (D.6a)-(D.6d) in a natural spinorial basis.

Let us start with (D.6a). By construction all terms have opposite chirality with respect to $\eta_{1}$. As discussed in Appendix B, we can expand it in the basis $\gamma_{a} \eta_{1}$ with $a=1, \ldots, 8$. The different components are obtained by contraction it with $\eta_{1}^{T} \gamma_{a}$. Using the definition of $\Phi$ (2.27), we can write

$$
\sqrt{2} \sin ^{2} \theta\left(e^{-2 D} \mathcal{D} e^{2 D}-\dot{\beta}-h_{\underline{u v}}\right)-\frac{1}{2} e^{\phi}\left(\iota_{a} f \cdot \Phi+f \cdot \iota_{a} \Phi\right) \mathrm{d} x^{a}=0 .
$$

We can repeat the same procedure, expanding (D.6b) in the basis $\gamma_{a} \eta_{2}$,

$$
\sqrt{2} \cos ^{2} \theta\left(e^{-2 D} \mathcal{D} e^{2 D}-\dot{\beta}+h_{\underline{u v}}\right)+\frac{1}{2} e^{\phi}\left(\iota_{a} f \cdot \Phi-f \cdot \iota_{a} \Phi\right) \mathrm{d} x^{a}=0 .
$$

(D.7) and (D.8) combine into the following algebraic equations

$$
\begin{aligned}
& e^{-2 D} \mathcal{D} e^{2 D}-\dot{\beta}+\cos 2 \theta h_{\underline{u v}}=\frac{1}{\sqrt{2}} e^{\phi}\left(f \cdot \iota_{a} \Phi\right) \mathrm{d} x^{a}, \\
& h_{\underline{u v}}+\cos 2 \theta\left(e^{-2 D} \mathcal{D} e^{2 D}-\dot{\beta}\right)=-\frac{1}{\sqrt{2}} e^{\phi}\left(\iota_{a} f \cdot \Phi\right) \mathrm{d} x^{a},
\end{aligned}
$$

which can be further simplified if one uses the supersymmetry equations (2.6). Indeed (2.6a) allows to determine some components of the NSNS flux

$$
\begin{aligned}
& h_{\underline{u}}=\cos 2 \theta e^{2 D} \mathcal{D} \beta, \\
& h_{\underline{u v}}=\cos 2 \theta \dot{\beta}-e^{-2 D} \mathcal{D}\left(\cos 2 \theta e^{2 D}\right),
\end{aligned}
$$

while (2.6b) gives

$$
\begin{aligned}
& f_{\underline{u}}=\sqrt{2} e^{2 D-\phi} \mathcal{D} \beta \wedge \Phi, \\
& f_{\underline{u v}}+\cos 2 \theta f=-\sqrt{2} e^{-\phi}\left[e^{-2 D+\phi} \mathcal{D}\left(e^{2 D-\phi} \Phi\right)-h \wedge \Phi-\dot{\beta} \wedge \Phi\right] .
\end{aligned}
$$

In particular, using (D.10), we can rewrite (D.9) as

$$
\begin{aligned}
& e^{-2 D} \mathcal{D} e^{2 D}-\dot{\beta}+\mathcal{D} \log (\sin 2 \theta)=\frac{1}{\sqrt{2} \sin ^{2}(2 \theta)} e^{\phi}\left(f \cdot \iota_{a} \Phi\right) \mathrm{d} x^{a}, \\
& \mathcal{D}_{a} \cos 2 \theta=\frac{1}{\sqrt{2}} e^{\phi} \iota_{a} f \cdot \Phi, \\
& \frac{\mathrm{d}}{\mathrm{d} v}(\cos 2 \theta)=\frac{\sqrt{2}}{4} e^{2 D+\phi} f_{\underline{v}} \cdot \Phi .
\end{aligned}
$$

Let us now pass to the last two equations, (D.6c) and (D.6d). We can expand them in the basis $\left(\eta_{1}, \gamma_{a b} \eta_{1}\right)$ and $\left(\eta_{2}, \gamma_{a b} \eta_{2}\right)$, respectively. Let us first contract (D.6c) with $\eta_{1}^{T}$. Recalling that we set $\eta_{1}^{T} \eta_{1}=\sqrt{2} \sin ^{2} \theta$, we get

$$
\frac{1}{\sqrt{2}} \frac{\mathrm{d}}{\mathrm{d} v}\left(\sin ^{2} \theta\right)+\frac{1}{8} e^{2 D+\phi} f_{\underline{v}} \cdot \Phi=0 .
$$


On the other hand, contracting (D.6d) with $\eta_{2}^{T}$ and using $\eta_{1}^{T} \eta_{1}=\sqrt{2} \cos ^{2} \theta$ gives

$$
\frac{1}{\sqrt{2}} \frac{\mathrm{d}}{\mathrm{d} v}\left(\cos ^{2} \theta\right)-\frac{1}{8} e^{2 D+\phi} f_{\underline{v}} \cdot \Phi=0 .
$$

These two equations are clearly equivalent and can be rewritten as

$$
\frac{1}{\sqrt{2}} \frac{\mathrm{d}}{\mathrm{d} v}(\cos 2 \theta)-\frac{1}{4} e^{2 D+\phi} f_{\underline{v}} \cdot \Phi=0 .
$$

We now have to consider the $\gamma_{a b} \eta_{1}$ and $\gamma_{a b} \eta_{2}$ components of (D.6c) and (D.6d). Let us introduce the four-forms $\Omega^{(1)}$ and $\Omega^{(2)}$ defined as

$$
\Omega_{a b c d}^{(1)}=\eta_{1}^{T} \gamma_{a b c d} \eta_{1}, \quad \Omega_{a b c d}^{(2)}=\eta_{2}^{T} \gamma_{a b c d} \eta_{2} .
$$

Then, by using (B.11) we can write

$$
\begin{aligned}
& \eta_{1}^{T} \gamma_{a b} \dot{\eta}_{1}-\frac{1}{2} \eta_{1}^{T} \gamma_{a b} m_{\mathrm{A}} \eta_{1}=-\frac{1}{8 \sqrt{2} \sin ^{2} \theta} \iota_{[a} \Omega^{(1)} \cdot \frac{\mathrm{d}}{\mathrm{d} v}\left(\iota_{b]} \Omega^{(1)}\right), \\
& \eta_{2}^{T} \gamma_{a b} \dot{\eta}_{2}-\frac{1}{2} \eta_{2}^{T} \gamma_{a b} m_{\mathrm{A}} \eta_{2}=-\frac{1}{8 \sqrt{2} \cos ^{2} \theta} \iota_{[a} \Omega^{(2)} \cdot \frac{\mathrm{d}}{\mathrm{d} v}\left(\iota_{b]} \Omega^{(2)}\right) .
\end{aligned}
$$

On the other hand, by using the projectors on the representations 7 of the two reduced Spin(7) structure group defined by $\eta_{1}$ and $\eta_{2}$,

$$
\begin{aligned}
& \left(P_{7}^{(1)}\right)_{a b}{ }^{c d}=\frac{1}{4}\left(\delta_{[a}^{[c} \delta_{b]}^{d]}-\frac{1}{2 \sqrt{2} \sin ^{2} \theta} \Omega_{a b}^{(1) c d}\right), \\
& \left(P_{7}^{(2)}\right)_{a b}{ }^{c d}=\frac{1}{4}\left(\delta_{[a}^{[c} \delta_{b]}^{d]}-\frac{1}{2 \sqrt{2} \cos ^{2} \theta} \Omega_{a b}^{(2) c d}\right),
\end{aligned}
$$

we can write

$$
\begin{aligned}
& \eta_{1} \gamma_{a b}(\mathcal{D} \omega+W \mathcal{D} \beta) \eta_{1}-\eta_{1} h_{\underline{v}} \eta_{1}=-4 \sqrt{2} \sin ^{2} \theta\left(P_{7}^{(1)}\right)_{a b}{ }^{c d}\left(\mathcal{D} \omega+W \mathcal{D} \beta-h_{\underline{v}}\right)_{c d}, \\
& \eta_{2} \gamma_{a b}(\mathcal{D} \omega+W \mathcal{D} \beta) \eta_{2}+\eta_{2} h_{\underline{v}} \eta_{2}=-4 \sqrt{2} \cos ^{2} \theta\left(P_{7}^{(2)}\right)_{a b}{ }^{c d}\left(\mathcal{D} \omega+W \mathcal{D} \beta+h_{\underline{v}}\right)_{c d} .
\end{aligned}
$$

Furthermore we have

$$
\begin{aligned}
& \eta_{1}^{T} \gamma_{a b} f_{\underline{v}} \eta_{2}=\Phi \cdot\left(\gamma_{a b} f_{\underline{v}}\right), \\
& \eta_{2}^{T} \gamma_{a b} \lambda\left(f_{\underline{v}}\right) \eta_{1}=-\Phi \cdot\left(f_{\underline{v}} \gamma_{a b}\right),
\end{aligned}
$$

where the action of gamma matrices on differential forms is explained after (2.31) and in the present case is explicitly given by

$$
\begin{aligned}
& \gamma_{a b} f_{\underline{v}}=\iota_{a} \iota_{b} f_{\underline{v}}+\mathrm{d} x_{a} \wedge \mathrm{d} x_{b} \wedge f_{\underline{v}}+2 \mathrm{~d} x_{[a} \wedge \iota_{b]} f_{\underline{v}}, \\
& f_{\underline{v}} \gamma_{a b}=\iota_{a} \iota_{b} f_{\underline{v}}+\mathrm{d} x_{a} \wedge \mathrm{d} x_{b} \wedge f_{\underline{v}}-2 \mathrm{~d} x_{[a} \wedge \iota_{b]} f_{\underline{v}},
\end{aligned}
$$

with $\mathrm{d} x_{a} \equiv g_{a b} \mathrm{~d} x^{b}$. Putting all these steps together, we obtain the following equations

$$
\begin{aligned}
\iota_{[a} \Omega^{(1)} \cdot \frac{\mathrm{d}}{\mathrm{d} v}\left(\iota_{b]} \Omega^{(1)}\right)= & -16 \sin ^{4} \theta e^{2 D}\left(P_{7}^{(1)}\right)_{a b}{ }^{c d}\left(\mathcal{D} \omega+W \mathcal{D} \beta-h_{\underline{v}}\right)_{c d} \\
& +\sqrt{2} \sin ^{2} \theta e^{2 D+\phi} \Phi \cdot\left(\gamma_{a b} f_{\underline{v}}\right), \\
\iota_{[a} \Omega^{(2)} \cdot \frac{\mathrm{d}}{\mathrm{d} v}\left(\iota_{b]} \Omega^{(2)}\right)= & -16 \sin ^{4} \theta e^{2 D}\left(P_{7}^{(2)}\right)_{a b}{ }^{c d}\left(\mathcal{D} \omega+W \mathcal{D} \beta+h_{\underline{v}}\right)_{c d} \\
& +\sqrt{2} \sin ^{2} \theta e^{2 D+\phi} \Phi \cdot\left(f_{\underline{v}} \gamma_{a b}\right) .
\end{aligned}
$$




\section{Appendix E. Derivation of the general ansatz of Section 3.2}

In this appendix we show how the ansatz we use in Section 3.2 to describe bound states of D1-D5-P branes is the most general one compatible with the BPS equations of 2.2.3 under some hypothesis we will discuss below.

We want to describe the back-reaction of bound states of D1-D5-P in $\mathbb{R}^{1,1} \times Y \times T^{4}$. The two assumptions we make are that the backgrounds are homogeneous and isotropic on $T^{4}$. Therefore all the fields in the ansatz only depend on the $\left(u, v, x^{i}\right)$ coordinates of $\mathbb{R}^{1,1} \times Y$ and we impose that $H$ has legs just along $\mathbb{R}^{1,1} \times Y$ and that the RR-flux polyform $F$ splits as follows:

$$
F_{\text {tot }}=F+\widehat{\operatorname{vol}}_{4} \wedge \tilde{F},
$$

where $F$ and $\tilde{F}$ have legs along $\mathbb{R}^{1,1} \times Y$ only. The ten-dimensional self-duality condition reduces to the six-dimensional conditions

$$
\tilde{F}=e^{4 \hat{G}} *_{6} \lambda(F),
$$

where $*_{6}$ uses the complete, warped, six-dimensional metric. Moreover $F$ and $\tilde{F}$ must satisfy the following six-dimensional Bianchi identities/equations of motion

$$
\begin{aligned}
& \mathrm{d}_{H} F=0, \\
& \mathrm{~d}_{H} \tilde{F}=0,
\end{aligned}
$$

where $\mathrm{d}_{H} \equiv \mathrm{d}-H$. In this appendix we will adapt the flux decomposition (2.16) to the metric (3.1). With obvious notation, we can write

$$
\begin{aligned}
& F=E^{\underline{u}} \wedge E^{\underline{v}} \wedge f_{\underline{u v}}+E^{\underline{v}} \wedge f_{\underline{v}}+E^{\underline{u}} \wedge f_{\underline{u}}+f, \\
& \tilde{F}=E^{\underline{u}} \wedge E^{\underline{v}} \wedge \tilde{f}_{\underline{u v}}+E^{\underline{v}} \wedge \tilde{f}_{\underline{v}}+E^{\underline{u}} \wedge \tilde{f}_{\underline{u}}+\tilde{f} .
\end{aligned}
$$

Notice that here $f \ldots$ denote the flux components along the four-dimensional space $Y$ and not, as in the rest of the paper, the eight-dimensional components transverse to $(u, v)$.

The duality condition (E.2) splits into the following set of duality conditions on $Y$ :

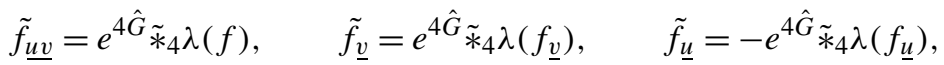

$$
\begin{aligned}
& \tilde{f}=e^{4 \hat{G}} \tilde{*}_{4} \lambda\left(f_{\underline{u v}}\right),
\end{aligned}
$$

where $\tilde{*}_{4}$ uses the complete warped four-dimensional metric $e^{2 G} \mathrm{~d} s_{4}^{2}$.

We will now solve the BPS conditions summarised in Section 2.2.3.

\section{E.1. Equation (2.36a)}

Let us start with $(2.36 \mathrm{a})$

$$
\mathrm{d} \chi=\iota_{K} H \text {. }
$$

As already discussed in Section 3.1, the most general local solution is provided by ${ }^{22}$

22 More precisely, the most general local solution takes the form $H=\mathrm{d} B$, with $B u$-independent and such that $\iota_{K} B=$ $-\chi+\mathrm{d} \lambda$ where $\lambda$ is a function. On the other hand, we can perform a gauge transformation $B \rightarrow B+\mathrm{d}(\lambda \mathrm{d} u)$ which allows to bring $\iota_{K} B$ into the form $\iota_{K} B=-\chi$. 


$$
B=-e^{2 D} \cos 2 \theta(\mathrm{d} u+\omega) \wedge(\mathrm{d} v+\beta)+b \wedge(\mathrm{d} v+\beta)+\mathcal{B},
$$

where $b=b_{i} \mathrm{~d} x^{i}$ and $\mathcal{B}=\frac{1}{2} \mathcal{B}_{i j} \mathrm{~d} x^{i} \wedge \mathrm{d} x^{j}$ have legs just along $Y$. Then

$$
\begin{aligned}
H= & -(\mathrm{d} u+\omega) \wedge(\mathrm{d} y+\beta) \wedge\left[\mathcal{D}\left(e^{2 D} \cos 2 \theta\right)-e^{2 D} \cos 2 \theta \dot{\beta}\right] \\
& +(\mathrm{d} v+\beta) \wedge\left[\dot{\mathcal{B}}-e^{2 D} \cos 2 \theta \mathcal{D} \omega-(\mathcal{D} b-\dot{\beta} \wedge b)\right] \\
& +(\mathrm{d} u+\omega) \wedge\left(e^{2 D} \cos 2 \theta \mathcal{D} \beta\right)+\mathcal{D} \mathcal{B}-\mathcal{D} \beta \wedge b
\end{aligned}
$$

\section{E.2. Equation (2.36b)}

We then pass to (2.36b). The isotropy condition (E.1) implies that the 'non-isotropic' components of $\mathrm{d}_{H}\left(e^{-\phi} \Psi\right)$ should vanish. Explicitly, we have

$$
\left.\mathrm{d}_{H}\left(e^{-\phi} \Psi\right)\right|_{\text {non-is. }}=-\mathrm{d}_{H}\left[e^{2(D+G+\hat{G})-\phi} \sin 2 \theta(\mathrm{d} v+\beta) \wedge \sum_{A=1}^{3} J_{A} \wedge \hat{J}_{A}\right]
$$

We can use the gauge freedom (3.2) to fix

$$
e^{-2 G}=e^{2(D+\hat{G})-\phi} \sin 2 \theta .
$$

With this choice, by using (E.8) we have

$$
\begin{aligned}
\left.\mathrm{d}_{H}\left(e^{-\phi} \Psi\right)\right|_{\text {non-is. }}= & \sum_{A=1}^{3} \hat{J}_{A} \wedge\left\{(\mathrm{d} v+\beta) \wedge\left(\mathcal{D} J_{A}-\dot{\beta} \wedge J_{A}\right)-\mathcal{D} \beta \wedge J_{A}\right. \\
& \left.+(\mathrm{d} u+\omega) \wedge(\mathrm{d} v+\beta) \wedge\left[e^{2 D} \cos 2 \theta \mathcal{D} \beta \wedge J_{A}\right]\right\} .
\end{aligned}
$$

Hence, $\left.\mathrm{d}_{H}\left(e^{-\phi} \Psi\right)\right|_{\text {non-is. }}=0$ gives the conditions

$$
\begin{aligned}
& \mathcal{D} J_{a}-\dot{\beta} \wedge J_{A}=0, \\
& \mathcal{D} \beta \wedge J_{A}=0 \quad \Leftrightarrow \quad *_{4} \mathcal{D} \beta=\mathcal{D} \beta,
\end{aligned}
$$

where now $*_{4}$ denote the four-dimensional Hodge star with respect to the metric without warpfactor. The first condition tells us that the non-trivial $v$-dependence of the background constitutes a potential obstruction to the integrability of the almost hyper-Kähler structure on $Y$.

Once we have imposed (E.10) and (E.12), the remaining equations contained in (2.36b) split as follows:

$$
\begin{aligned}
& \mathrm{d}_{H}\left(e^{-\phi} \varphi\right)=\iota_{K} F+\chi \wedge F, \\
& \mathrm{~d}_{H}\left(e^{4 \hat{G}-\phi} \varphi\right)=\iota_{K} \tilde{F}+\chi \wedge \tilde{F},
\end{aligned}
$$

where $\varphi$ is the part of the $\Psi$ with all legs in $\mathbb{R}^{1,1} \times Y$ :

$$
\varphi=e^{2 D} \sin 2 \theta(\mathrm{d} v+\beta) \wedge\left(1+e^{4 G} \operatorname{vol}_{4}\right) .
$$

Using (E.6) and $\mathcal{L}_{k} B=0$, (E.13a) can be rewritten as

$$
\mathrm{d}\left(e^{-\phi} e^{B} \varphi\right)=\iota_{K}\left(e^{B} F\right)
$$

and can therefore be solved in the same way as (E.6). Locally we can write $F$ as

$$
F=\mathrm{d}_{H} C=e^{-B} \mathrm{~d}\left(e^{B} C\right),
$$


where $C=C_{0}+C_{2}+C_{4}$ is $u$-independent. $C$ must satisfy $\iota_{K}\left(e^{B} C\right)=-e^{-\phi} e^{B} \varphi$ and then

$$
\iota_{K} C=-e^{-\phi} \varphi-\chi \wedge C,
$$

which means that we can set

$$
\begin{aligned}
C & =-(\mathrm{d} u+\omega) \wedge\left(e^{-\phi} \varphi+\chi \wedge \mathcal{C}\right)+c \wedge(\mathrm{d} v+\beta)+\mathcal{C} \\
& =-e^{2 D}(\mathrm{~d} u+\omega) \wedge(\mathrm{d} v+\beta) \wedge\left[e^{-\phi} \sin 2 \theta+\cos 2 \theta \mathcal{C}\right]+c \wedge(\mathrm{d} v+\beta)+\mathcal{C},
\end{aligned}
$$

where $c \equiv c_{1}+c_{3}$ and $\mathcal{C} \equiv \mathcal{C}_{0}+\mathcal{C}_{2}+\mathcal{C}_{4}$ are polyforms with legs along $Y$ and in the second

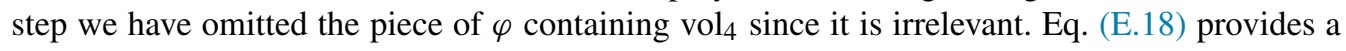
parametrisation of the most general local solution of (E.13a).

The most general solution of (E.13b) can be obtained in an almost identical way. Setting locally $\tilde{F}=\mathrm{d}_{H} \tilde{C}$, with $\tilde{C}=\tilde{C}_{0}+\tilde{C}_{2}+\tilde{C}_{4}$ gives the general local solution

$$
\begin{aligned}
\tilde{C} & =-(\mathrm{d} u+\omega) \wedge\left(e^{4 \hat{G}-\phi} \varphi+\chi \wedge \tilde{\mathcal{C}}\right)+\tilde{c} \wedge(\mathrm{d} v+\beta)+\tilde{\mathcal{C}} \\
& =-e^{2 D}(\mathrm{~d} u+\omega) \wedge(\mathrm{d} v+\beta) \wedge\left(e^{4 \hat{G}-\phi} \sin 2 \theta+\cos 2 \theta \tilde{\mathcal{C}}\right)+\tilde{c} \wedge(\mathrm{d} v+\beta)+\tilde{\mathcal{C}},
\end{aligned}
$$

where again $\tilde{c} \equiv \tilde{c}_{1}+\tilde{c}_{3}$ and $\tilde{\mathcal{C}} \equiv \tilde{\mathcal{C}}_{0}+\tilde{\mathcal{C}}_{2}+\tilde{\mathcal{C}}_{4}$ have legs along $Y$, and, in the last line, we have omitted an irrelevant six-form.

Now that we found two independent general solutions for (E.3a) and (E.3b), we have to compute $F=\mathrm{d}_{H} C$ and $\tilde{F}=\mathrm{d}_{H} \tilde{C}$ and impose the self-duality condition (E.2). In the notation of (E.4a) and (E.4b), we have for $F$

$$
\begin{aligned}
f_{\underline{u v}}= & -e^{-2 D} \mathcal{D}\left(e^{2 D-\phi} \sin 2 \theta\right)-\cos 2 \theta(\mathcal{D C}-\mathcal{D} \beta \wedge c) \\
& +e^{-\phi} \sin 2 \theta \dot{\beta}+(\mathcal{D B}-\mathcal{D} \beta \wedge b) \wedge\left(e^{-\phi} \sin 2 \theta+\cos 2 \theta \mathcal{C}\right), \\
f_{\underline{v}}= & e^{-2 D}\left[\dot{\mathcal{C}}-\dot{\mathcal{B}} \wedge \mathcal{C}-e^{2 D-\phi} \sin 2 \theta(\mathcal{D} \omega+W \mathcal{D} \beta)+(\mathcal{D} c-\dot{\beta} \wedge c)\right. \\
& -(\mathcal{D} b-\dot{\beta} \wedge b) \wedge \mathcal{C}-(\mathcal{D B}-\mathcal{D} \beta \wedge b) \wedge c], \\
f_{\underline{u}}= & e^{2 D-\phi} \sin 2 \theta \mathcal{D} \beta, \\
f= & (\mathcal{D C}-\mathcal{D} \beta \wedge c)-(\mathcal{D B}-\mathcal{D} \beta \wedge b) \wedge \mathcal{C},
\end{aligned}
$$

and for $\tilde{F}$

$$
\begin{aligned}
& \tilde{f}_{\underline{u v}}=-e^{-2 D} \mathcal{D}\left(e^{2 D+4 \hat{G}-\phi} \sin 2 \theta\right)-\cos 2 \theta(\mathcal{D} \tilde{\mathcal{C}}-\mathcal{D} \beta \wedge \tilde{c}) \\
&+e^{4 \hat{G}-\phi} \sin 2 \theta \dot{\beta}+(\mathcal{D B}-\mathcal{D} \beta \wedge b) \wedge\left(e^{4 \hat{G}-\phi} \sin 2 \theta+\cos 2 \theta \tilde{\mathcal{C}}\right) \\
& \tilde{f}_{\underline{v}}= e^{-2 D}\left[\dot{\tilde{\mathcal{C}}}+\dot{\mathcal{B}} \wedge \tilde{\mathcal{C}}-e^{2 D+4 \hat{G}-\phi} \sin 2 \theta(\mathcal{D} \omega+W \mathcal{D} \beta)+(\mathcal{D} \tilde{c}-\dot{\beta} \wedge \tilde{c})\right. \\
&-(\mathcal{D} b-\dot{\beta} \wedge b) \wedge \tilde{\mathcal{C}}-(\mathcal{D B}-\mathcal{D} \beta \wedge b) \wedge \tilde{c}] \\
& \tilde{f}_{\underline{u}}= e^{2 D+4 G-\phi} \sin 2 \theta \mathcal{D} \beta \\
& \tilde{f}=(\mathcal{D} \tilde{\mathcal{C}}-\mathcal{D} \beta \wedge \tilde{c})-(\mathcal{D B}-\mathcal{D} \beta \wedge b) \wedge \tilde{\mathcal{C}} .
\end{aligned}
$$

\section{E.3. Equations (2.37a) and (2.37b)}

Let us start from (2.37a). Using the duality conditions (E.5) and the expressions for the fluxes (E.20), (E.21), this becomes 


$$
\mathcal{D}(2 \hat{G}-\phi)+\frac{1}{2} e^{\phi} \frac{\cos 2 \theta}{\sin 2 \theta}\left(\mathcal{D} \mathcal{C}_{0}+e^{-4 \hat{G}} \mathcal{D} \tilde{\mathcal{C}}_{0}\right)=0 .
$$

To arrive at this expression we have rewritten the right-hand side of (2.37a) as

$$
\begin{aligned}
\frac{1}{\sqrt{2} \sin ^{2}(2 \theta)} e^{\phi}\left(f \cdot \iota_{a} \Phi\right) \mathrm{d} x^{a} & =\frac{e^{\phi}}{2 \sin 2 \theta}\left(\tilde{*}_{4} f_{3}+e^{-4 \hat{G}} \tilde{*}_{4} \tilde{f}_{3}\right) \\
& =-\frac{e^{\phi}}{2 \sin 2 \theta}\left(e^{-4 \hat{G}} \tilde{f}_{\underline{u v} 1}+f_{\underline{u v} 1}\right) .
\end{aligned}
$$

Let us now look at (2.37b). The r.h.s. is

$$
\frac{1}{\sqrt{2}} e^{\phi} \iota_{a} f \cdot \Phi=\frac{\sin 2 \theta}{2} e^{\phi}\left(f_{1}+e^{-4 \hat{G}} \tilde{f}_{1}\right),
$$

and hence, substituting the expressions for $f$ and $\tilde{f}$, that equation reduces to

$$
\mathcal{D} \cos 2 \theta=\frac{1}{2} \sin 2 \theta e^{\phi}\left(\mathcal{D C} \mathcal{C}_{0}+e^{-4 \hat{G}} \mathcal{D} \tilde{\mathcal{C}}_{0}\right)
$$

Combining (E.22) and (E.25) one obtains

$$
\mathcal{D}(2 \hat{G}-\phi-\log \sin 2 \theta)=0,
$$

which implies

$$
2 \hat{G}-\phi-\log \sin 2 \theta=0,
$$

up to an irrelevant constant which can be reabsorbed by a redefinition of the dilaton. The relation above and the gauge choice (E.10) leave only two independent quantities out of the three warp factors $D, G, \hat{G}$ and the dilaton $\phi$. In the following, we choose to keep as independent quantities $G$ and $\hat{G}$, so that

$$
e^{\phi}=\frac{e^{2 \hat{G}}}{\sin 2 \theta}, \quad e^{D}=\frac{e^{-G}}{\sin 2 \theta} .
$$

The remaining two scalars, $C_{0}$ and $\tilde{C}_{0}$, are not completely fixed at this point but are related by the relation (E.25), which can be rewritten as

$$
\mathcal{D} \mathcal{C}_{0}+e^{-4 \hat{G}} \mathcal{D} \tilde{\mathcal{C}}_{0}=2 e^{-2 \hat{G}} \mathcal{D} \cos 2 \theta .
$$

\section{E.4. Self-duality of the RR field strengths}

Let us now examine the duality relations (E.5). First, notice that by using (E.12b) the third condition of (E.5) is automatically satisfied. The other conditions

$$
\tilde{f}_{\underline{u v}}=e^{4 \hat{G}} \tilde{*}_{4} \lambda(f), \quad \tilde{f}=e^{4 \hat{G}_{\tilde{*}_{4}} \lambda\left(f_{\underline{u v}}\right),}
$$

once expanded in forms of fixed degree, can be written as

$$
\begin{aligned}
\mathcal{D} \mathcal{C}_{2}-\mathcal{D} \beta \wedge c_{1}= & e^{2(G-\hat{G})} *_{4}\left[\left(e^{-2 \hat{G}} \cos 2 \theta-\mathcal{C}_{0}\right) \mathcal{D} \tilde{\mathcal{C}}_{0}\right. \\
& \left.+\left(e^{2 \hat{G}} \cos 2 \theta \mathcal{C}_{0}+\sin ^{2} 2 \theta\right)(\dot{\beta}-2 \mathcal{D}(\hat{G}-G))\right], \\
\mathcal{D} \tilde{\mathcal{C}}_{2}-\mathcal{D} \beta \wedge \tilde{\mathcal{C}}_{1}= & e^{2(G+\hat{G})} *_{4}\left[\left(e^{2 \hat{G}} \cos 2 \theta-\tilde{\mathcal{C}}_{0}\right) \mathcal{D} \mathcal{C}_{0}\right. \\
& \left.+\left(e^{-2 \hat{G}} \cos 2 \theta \tilde{\mathcal{C}}_{0}+\sin ^{2} 2 \theta\right)(\dot{\beta}+2 \mathcal{D}(\hat{G}+G))\right],
\end{aligned}
$$




$$
\begin{aligned}
& \mathcal{D B}-\mathcal{D} \beta \wedge b=*_{4}\left[e^{2(G-\hat{G})} \mathcal{D} \tilde{C}_{0}+e^{2 G} \cos 2 \theta(\dot{\beta}-2 \mathcal{D}(\hat{G}-G))\right], \\
& \mathcal{D} \mathcal{C}_{0}-e^{-4 \hat{G}} \mathcal{D} \tilde{\mathcal{C}_{0}}=-4 e^{-2 \hat{G}} \cos 2 \theta \mathcal{D} \hat{G}
\end{aligned}
$$

The last equation, (E.31d), provides another relation on the scalars in the ansatz, which, combined with (E.29) gives

$$
\mathcal{D}\left(\mathcal{C}_{0}-e^{-2 \hat{G}} \cos 2 \theta\right)=0, \quad \mathcal{D}\left(\tilde{\mathcal{C}}_{0}-e^{2 \hat{G}} \cos 2 \theta\right)=0
$$

and hence, up to a constant,

$$
\mathcal{C}_{0}=e^{-2 \hat{G}} \cos 2 \theta, \quad \tilde{\mathcal{C}}_{0}=e^{2 \hat{G}} \cos 2 \theta .
$$

These relations can be used to simplify the previous ones

$$
\begin{aligned}
& \mathcal{D} \mathcal{C}_{2}-\mathcal{D} \beta \wedge c_{1}=*_{4}\left[e^{2(G-\hat{G})} \dot{\beta}+\mathcal{D} e^{2(G-\hat{G})}\right], \\
& \mathcal{D} \tilde{\mathcal{C}}_{2}-\mathcal{D} \beta \wedge \tilde{c}_{1}=*_{4}\left[e^{2(G+\hat{G})} \dot{\beta}+\mathcal{D} e^{2(G+\hat{G})}\right], \\
& \mathcal{D} \mathcal{B}-\mathcal{D} \beta \wedge b=*_{4}\left[e^{2 G} \cos 2 \theta \dot{\beta}+\mathcal{D}\left(e^{2 G} \cos 2 \theta\right)\right] .
\end{aligned}
$$

Let us now consider the last non-trivial duality constraint in (E.5)

$$
\tilde{f}_{\underline{v}}=e^{4 G} \tilde{*}_{4} \lambda\left(f_{\underline{v}}\right) \text {. }
$$

To this extent it is convenient to introduce some more notation, the three two-forms

$$
\begin{aligned}
& \Theta=\dot{\mathcal{C}}_{2}+\mathcal{D} c_{1}-\dot{\beta} \wedge c_{1}, \\
& \tilde{\Theta}=\dot{\tilde{\mathcal{C}}}_{2}+\mathcal{D} \tilde{c}_{1}-\dot{\beta} \wedge \tilde{c}_{1}, \\
& \Theta_{b}=\dot{\mathcal{B}}+\mathcal{D} b-\dot{\beta} \wedge b .
\end{aligned}
$$

Then the self-duality constraints read

$$
\begin{aligned}
\frac{\mathrm{d}}{\mathrm{d} v}\left(e^{2 \hat{G}} \cos 2 \theta\right)= & e^{4(\hat{G}-G)} *_{4}\left[\dot{\mathcal{C}}_{4}-\Theta_{b} \wedge \mathcal{C}_{2}+\left(\mathcal{D} c_{3}-\dot{\beta} \wedge c_{3}\right)\right. \\
& \left.-(\mathcal{D B}-\mathcal{D} \beta \wedge b) \wedge c_{1}\right], \\
\frac{\mathrm{d}}{\mathrm{d} v}\left(e^{-2 \hat{G}} \cos 2 \theta\right)= & e^{-4(\hat{G}+G)} *_{4}\left[\dot{\tilde{\mathcal{C}}}_{4}-\Theta_{b} \wedge \tilde{\mathcal{C}}_{2}+\left(\mathcal{D} \tilde{c}_{3}-\dot{\beta} \wedge \tilde{c}_{3}\right)\right. \\
& \left.-(\mathcal{D B}-\mathcal{D} \beta \wedge b) \wedge \tilde{c}_{1}\right],
\end{aligned}
$$

and

$$
\mathcal{D} \omega+*_{4} \mathcal{D} \omega+2 W \mathcal{D} \beta=e^{2(G-\hat{G})} \tilde{\Theta}+e^{2(G+\hat{G})} *_{4} \Theta-e^{2 G} \cos 2 \theta\left(\Theta_{b}+*_{4} \Theta_{b}\right) .
$$

\section{E.5. Equation $(2.37 c)$}

Using the expression for the fluxes (E.20) and the self-duality conditions, the supersymmetry constraint $(2.37 \mathrm{c})$ becomes

$$
\begin{aligned}
\frac{\mathrm{d}}{\mathrm{d} v}(\cos 2 \theta) & =\frac{\sqrt{2}}{4} e^{2 D+\phi} f_{\underline{v}} \cdot \Phi \\
& =\frac{1}{2} \sin 2 \theta e^{\phi}\left(\dot{\mathcal{C}}_{0}+e^{-4 \hat{G}} \dot{\tilde{\mathcal{C}}}_{0}\right),
\end{aligned}
$$

which is identically satisfied by the relations (E.33). 


\section{E.6. Equations (2.37d) and (2.37e)}

The last equations to analyse are (2.37d) and (2.37e). As shown in Section 3.1, the requirement of having supersymmetric D1-D5-P systems forces the two eight-dimensional spinors $\eta_{1}$ and $\eta_{2}$ to be proportional

$$
\eta_{1}=2^{1 / 4} \sin \theta \eta, \quad \eta_{2}=2^{1 / 4} \cos \theta \eta .
$$

Thus the two Spin(7) structures characterising the most general background with null $K$ collapse to a single one

$$
\Omega^{(1)}=\sqrt{2} \sin ^{2} \theta \Omega, \quad \Omega^{(2)}=\sqrt{2} \cos ^{2} \theta \Omega
$$

associated with the four-form (3.19)

$$
\Omega=e^{4 G} \text { vol }_{4}+e^{4 G} \widehat{\operatorname{vol}}_{4}-e^{2 G+2 \hat{G}} \sum_{A=1}^{3} J_{A} \wedge \hat{J}_{A},
$$

where $J_{A}$ and $\hat{J}_{A}$ define almost hyper-Kähler structures on $Y$ and $T^{4}$ respectively (see (3.21)).

This brings some simplifications to (2.37d) and (2.37e). In particular, their sum and difference give

$$
\begin{aligned}
& 16 \sqrt{2}\left(P_{7}\right)_{a b} c d\left(h_{\underline{v}}\right)_{c d}=-e^{\phi} \Phi \cdot\left(\frac{1}{\sin ^{2} \theta} \gamma_{a b} f_{\underline{v}}-\frac{1}{\cos ^{2} \theta} f_{\underline{v}} \gamma_{a b}\right), \\
& \iota_{[a} \Omega \cdot \frac{\mathrm{d}}{\mathrm{d} v}\left(\iota_{b]} \Omega\right)+8 e^{2 D}\left(P_{7}\right)_{a b} c d(\mathcal{D} \omega+W \mathcal{D} \beta)_{c d} \\
& =\frac{e^{2 D+\phi}}{2 \sqrt{2}} \Phi \cdot\left(\frac{1}{\sin ^{2} \theta} \gamma_{a b} f_{\underline{v}}+\frac{1}{\cos ^{2} \theta} f_{\underline{v}} \gamma_{a b}\right) .
\end{aligned}
$$

In order to simplify further the equations above, we need some extra work. Let us first introduce the two-form

$$
\psi \equiv \sum_{A} \psi_{A} J_{A}=\frac{1}{4} \epsilon^{A B C}\left(J_{A} \cdot \dot{J}_{B}\right) J_{C} \equiv \frac{1}{8} \epsilon^{A B C}\left(J_{A}\right)^{i j}\left(\dot{J}_{B}\right)_{i j} J_{C},
$$

which is anti-self-dual on $Y$. Notice that, for any self-dual and anti-self-dual 2-forms $\rho_{\text {sd }}$ and $\rho_{\text {asd }}$ we have

$$
\left(J_{A}\right)_{[i}^{k}\left(\rho_{\mathrm{sd}}\right)_{j] k} \equiv 0, \quad \rho_{\text {asd }} \equiv \frac{1}{2} \sum_{A}\left(J_{A} \cdot \rho_{\text {asd }}\right) J_{A} .
$$

These identities can be used to check that $\psi$ can be rewritten as

$$
\psi=-\frac{1}{4} \sum_{A}\left(J_{A}\right)_{i}{ }^{k}\left(\dot{J}_{A}\right)_{j k} \mathrm{~d} x^{i} \wedge \mathrm{d} x^{j} .
$$

Then, it is tedious but straightforward to prove that

$$
\begin{aligned}
& \iota_{[m} \Omega \cdot \frac{\mathrm{d}}{\mathrm{d} v} \iota_{n]} \Omega=-4 e^{2 \hat{G}} \sum_{A} \psi_{A}\left(\hat{J}_{A}\right)_{m n}, \\
& \iota_{[i} \Omega \cdot \frac{\mathrm{d}}{\mathrm{d} v} \iota_{j]} \Omega=-4 e^{2 G} \psi_{i j},
\end{aligned}
$$




$$
\begin{aligned}
& \left(P_{7}\right)_{a b}^{i j}(\mathcal{D} \omega+W \mathcal{D} \beta)_{i j}=\frac{1}{4} e^{2 \hat{G}-2 G} \sum_{A}\left(J_{A} \cdot \mathcal{D} \omega\right)\left(\hat{J}_{A}\right)_{a b}, \\
& \left(P_{7}\right)_{i j}{ }^{h k}(\mathcal{D} \omega+W \mathcal{D} \beta)_{h k}=\frac{1}{4}\left[\left(1-*_{4}\right) \mathcal{D} \omega\right]_{i j}, \\
& \left(P_{7}\right)_{a b}^{i j}\left(h_{\underline{v}}\right)_{i j}=-\frac{1}{4} e^{2 G-2 \tilde{G}} \sum_{A}\left(J_{A} \cdot h_{\underline{v}}\right)\left(\hat{J}_{A}\right)_{a b}, \\
& \left(P_{7}\right)_{i j}{ }^{h k}\left(h_{\underline{v}}\right)_{h k}=-\frac{1}{4}\left[\left(1-*_{4}\right) h_{\underline{v}}\right]_{i j}, \\
& \Phi \cdot\left(\gamma_{a b} f_{\underline{v}}^{\text {tot }}\right)=-\sqrt{2} \sin 2 \theta e^{2 \hat{G}-2 G} \sum_{A}\left(J_{A} \cdot f_{\underline{v} 2}\right)\left(\hat{J}_{A}\right)_{a b}, \\
& \Phi \cdot\left(f_{\underline{v}}^{\text {tot }} \gamma_{a b}\right)=-\sqrt{2} \sin 2 \theta e^{2 \hat{G}-2 G} \sum_{A}\left(J_{A} \cdot f_{\underline{v} 2}\right)\left(\hat{J}_{A}\right)_{a b}, \\
& \Phi \cdot\left(\gamma_{i j} f_{\underline{v}}^{\text {tot }}\right)=-\sqrt{2} \sin 2 \theta\left[\left(1-*_{4}\right) f_{\underline{v} 2}\right]_{i j}, \\
& \Phi \cdot\left(f_{\underline{v}}^{\text {tot }} \gamma_{i j}\right)=-\sqrt{2} \sin 2 \theta\left[\left(1-*_{4}\right) f_{\underline{v} 2}\right]_{i j},
\end{aligned}
$$

where, on the left-hand side, we introduced the superscript ${ }^{\text {tot }}$ to distinguish the eight-dimensional RR fluxes defined in (2.16) from the four-dimensional ones in (E.4a).

Using the identities (E.47) and the expression for the fluxes derived in the previous sections, it is possible to reduce the constraint (E.43a) to a more readable form

$$
\begin{aligned}
& \left(1-*_{4}\right) \Theta=2 e^{2 G-2 \hat{G}} \psi, \\
& \left(1-*_{4}\right) \Theta_{b}=2 \cos 2 \theta e^{2 G} \psi .
\end{aligned}
$$

Finally, combining the previous equations with self-duality condition (E.38) gives

$$
\left(1-*_{4}\right) \tilde{\Theta}=2 e^{2 G+2 \hat{G}} \psi .
$$

\section{E.7. Summary and relation with the notation of previous works}

In this section, we summarize our ansatz and the complete set of supergravity equations one has to solve to find supergravity solutions describing D1-D5-P systems. We change the notation to make it compatible with previous works $[16,33,38-40,44]$. We rename the various metric and gauge field coefficients as follows:

$$
\begin{array}{llll}
Z \rightarrow Z_{1}, & \tilde{Z} \rightarrow Z_{2}, & Z_{b} \rightarrow Z_{4}, & W \rightarrow \frac{\mathcal{F}}{2}, \\
c_{1} \rightarrow a_{1}, & \tilde{c}_{1} \rightarrow a_{2}, & b \rightarrow a_{4}, & \\
\mathcal{C}_{2} \rightarrow \gamma_{2}, & \tilde{\mathcal{C}}_{2} \rightarrow \gamma_{1}, & \mathcal{B} \rightarrow \delta_{2}, & c_{3} \rightarrow x_{3} .
\end{array}
$$

Note that now the number subscripts do not denote anymore the form degree but rather refer to the particular multiplet the corresponding fields belong to.

In the new notation the ansatz for the metric in string frame, the dilaton, the NSNS $B$-field and the RR gauge fields is 


$$
\begin{aligned}
& \mathrm{d} s_{(10)}^{2}=-\frac{2 \alpha}{\sqrt{Z_{1} Z_{2}}}(\mathrm{~d} v+\beta)\left[\mathrm{d} u+\omega+\frac{\mathcal{F}}{2}(\mathrm{~d} v+\beta)\right]+\sqrt{Z_{1} Z_{2}} \mathrm{~d} s_{4}^{2}+\sqrt{\frac{Z_{1}}{Z_{2}}} \mathrm{~d} \hat{s}_{4}^{2}, \\
& e^{2 \phi}=\alpha \frac{Z_{1}}{Z_{2}} \\
& B=-\frac{\alpha Z_{4}}{Z_{1} Z_{2}}(\mathrm{~d} u+\omega) \wedge(\mathrm{d} v+\beta)+a_{4} \wedge(\mathrm{d} v+\beta)+\delta_{2}, \\
& C_{0}=\frac{Z_{4}}{Z_{2}}, \\
& C_{2}=-\frac{\alpha}{Z_{1}}(\mathrm{~d} u+\omega) \wedge(\mathrm{d} v+\beta)+a_{1} \wedge(\mathrm{d} v+\beta)+\gamma_{2}, \\
& C_{4}=\frac{Z_{4}}{Z_{2}} \hat{v o l}_{4}-\frac{\alpha Z_{4}}{Z_{1} Z_{2}} \gamma_{2} \wedge(\mathrm{d} u+\omega) \wedge(\mathrm{d} v+\beta)+x_{3} \wedge(\mathrm{d} v+\beta),
\end{aligned}
$$

where

$$
\alpha=\frac{Z_{1} Z_{2}}{Z_{1} Z_{2}-Z_{4}^{2}} .
$$

As already mentioned at the end of Section 3.2, the search of a solution can be systematised by solving the equations in the following order

- Equations for $\mathrm{d} s_{4}^{2}, \beta$ :

$$
\begin{aligned}
& \mathrm{d} J_{A}=\frac{\mathrm{d}}{\mathrm{d} v}\left(\beta \wedge J_{A}\right), \quad *_{4} J_{A}=-J_{A}, \quad J_{A} \wedge J_{B}=-2 \delta_{A B} \text { vol }_{4}, \\
& *_{4} \mathcal{D} \beta=\mathcal{D} \beta
\end{aligned}
$$

- Equations for $Z_{1}, a_{2}, \gamma_{1}$ :

$$
\begin{aligned}
& *_{4}\left(\mathcal{D} Z_{1}+\dot{\beta} Z_{1}\right)=\mathcal{D} \gamma_{1}-a_{2} \wedge \mathcal{D} \beta, \\
& \Theta_{2}-Z_{1} \psi=*_{4}\left(\Theta_{2}-Z_{1} \psi\right) \quad \text { with } \Theta_{2}=\mathcal{D} a_{2}-\dot{\beta} \wedge a_{2}+\dot{\gamma}_{1}
\end{aligned}
$$

with

$$
\psi=\frac{1}{8} \epsilon^{A B C}\left(J_{A}\right)^{i j}\left(\dot{J}_{B}\right)_{i j} J_{C}
$$

- Equations for $Z_{2}, a_{1}, \gamma_{2}$ :

$$
\begin{aligned}
& *_{4}\left(\mathcal{D} Z_{2}+\dot{\beta} Z_{2}\right)=\mathcal{D} \gamma_{2}-a_{1} \wedge \mathcal{D} \beta \\
& \Theta_{1}-Z_{2} \psi=*_{4}\left(\Theta_{1}-Z_{2} \psi\right) \quad \text { with } \Theta_{1}=\mathcal{D} a_{1}-\dot{\beta} \wedge a_{1}+\dot{\gamma}_{2}
\end{aligned}
$$

- Equations for $Z_{4}, a_{4}, \delta_{2}$ :

$$
\begin{aligned}
& *_{4}\left(\mathcal{D} Z_{4}+\dot{\beta} Z_{4}\right)=\mathcal{D} \delta_{2}-a_{4} \wedge \mathcal{D} \beta, \\
& \Theta_{4}-Z_{4} \psi=*_{4}\left(\Theta_{4}-Z_{4} \psi\right) \quad \text { with } \Theta_{4}=\mathcal{D} a_{4}-\dot{\beta} \wedge a_{4}+\dot{\delta}_{2} ;
\end{aligned}
$$

- Equations for $\omega, \mathcal{F}$ : 


$$
\begin{aligned}
& \mathcal{D} \omega+*_{4} \mathcal{D} \omega+\mathcal{F} \mathcal{D} \beta=Z_{1} *_{4} \Theta_{1}+Z_{2} \Theta_{2}-Z_{4}\left(\Theta_{4}+*_{4} \Theta_{4}\right), \\
& \quad-\frac{\alpha}{4} \frac{\mathrm{d}}{\mathrm{d} v}\left(\frac{\sqrt{Z_{1} Z_{2}}}{\alpha^{2}} g^{i j}\right) \frac{\mathrm{d}}{\mathrm{d} v}\left(\sqrt{Z_{1} Z_{2}} g_{i j}\right)-\frac{1}{2} \frac{\sqrt{Z_{1} Z_{2}}}{\alpha} g^{i j} \frac{\mathrm{d}^{2}}{\mathrm{~d} v^{2}}\left(\sqrt{Z_{1} Z_{2}} g_{i j}\right) \\
& \left.+\sqrt{Z_{1} Z_{2}} \frac{\mathrm{d}}{\mathrm{d} v}\left[\sqrt{Z_{1} Z_{2}} \frac{\mathrm{d}}{\mathrm{d} v}\left(\frac{Z_{1} Z_{2}}{Z_{1} Z_{2}}\right)\right]-\frac{Z_{2}}{2} \frac{\mathrm{d}}{\mathrm{d} v}\left(\frac{Z_{1}}{Z_{2}}\right)\right]_{1}^{2}\left[\frac{\mathrm{d}}{\mathrm{d} v}\left(\frac{Z_{4}}{Z_{1}}\right)\right]^{2}-\frac{1}{2} Z_{2}^{2}\left[\frac{\mathrm{d}}{\mathrm{d} v}\left(\frac{Z_{4}}{Z_{2}}\right)\right]^{2} \\
& +\frac{1}{2} *_{4}\left[\left(\Theta_{1}-Z_{2} \psi\right) \wedge\left(\Theta_{2}-Z_{1} \psi\right)-\left(\Theta_{4}-Z_{4} \psi\right) \wedge\left(\Theta_{4}-Z_{4} \psi\right)\right. \\
& \left.+\frac{Z_{1} Z_{2}}{\alpha} \psi \wedge \psi-2 \psi \wedge \mathcal{D} \omega\right]=0
\end{aligned}
$$

with

$$
L=\dot{\omega}+\frac{\mathcal{F}}{2} \dot{\beta}-\frac{1}{2} \mathcal{D} \mathcal{F}
$$

- Equation for $x_{3}$ :

$$
\mathcal{D} x_{3}-\dot{\beta} \wedge x_{3}-\Theta_{4} \wedge \gamma_{2}+a_{1} \wedge\left(\mathcal{D} \delta_{2}-a_{4} \wedge \mathcal{D} \beta\right)=Z_{2}^{2} *_{4} \frac{\mathrm{d}}{\mathrm{d} v}\left(\frac{Z_{4}}{Z_{2}}\right) .
$$

Only the first set of Eqs. (E.53) is non-linear. The remaining conditions, if solved in the order presented above, represent a set of linear equations for their respective unknowns.

\section{References}

[1] N. Hitchin, Generalized Calabi-Yau manifolds, Quart. J. Math. Oxford Ser. 54 (2003) 281-308, arXiv:math/ 0209099.

[2] M. Gualtieri, Generalized complex geometry, arXiv:math/0401221.

[3] M. Grana, R. Minasian, M. Petrini, A. Tomasiello, Supersymmetric backgrounds from generalized Calabi-Yau manifolds, J. High Energy Phys. 0408 (2004) 046, arXiv:hep-th/0406137.

[4] M. Grana, R. Minasian, M. Petrini, A. Tomasiello, Generalized structures of $N=1$ vacua, J. High Energy Phys. 0511 (2005) 020, arXiv:hep-th/0505212.

[5] L. Martucci, P. Smyth, Supersymmetric D-branes and calibrations on general $N=1$ backgrounds, J. High Energy Phys. 0511 (2005) 048, arXiv:hep-th/0507099.

[6] L. Martucci, D-branes on general $N=1$ backgrounds: Superpotentials and D-terms, J. High Energy Phys. 0606 (2006) 033, arXiv:hep-th/0602129.

[7] J.P. Gauntlett, S. Pakis, The geometry of $D=11$ Killing spinors, J. High Energy Phys. 0304 (2003) 039, arXiv: hep-th/0212008.

[8] J.P. Gauntlett, D. Martelli, S. Pakis, D. Waldram, G structures and wrapped NS5-branes, Commun. Math. Phys. 247 (2004) 421-445, arXiv:hep-th/0205050.

[9] J.P. Gauntlett, J.B. Gutowski, S. Pakis, The geometry of $D=11$ null Killing spinors, J. High Energy Phys. 0312 (2003) 049, arXiv:hep-th/0311112.

[10] E.J. Hackett-Jones, D.J. Smith, Type IIB Killing spinors and calibrations, J. High Energy Phys. 0411 (2004) 029, arXiv:hep-th/0405098.

[11] P. Saffin, Type IIA Killing spinors and calibrations, Phys. Rev. D 71 (2005) 025018, arXiv:hep-th/0407156.

[12] A. Tomasiello, Generalized structures of ten-dimensional supersymmetric solutions, J. High Energy Phys. 1203 (2012) 073, arXiv:1109.2603 [hep-th].

[13] A. Strominger, C. Vafa, Microscopic origin of the Bekenstein-Hawking entropy, Phys. Lett. B 379 (1996) 99-104, arXiv:hep-th/9601029. 
[14] C.G. Callan, J.M. Maldacena, D-brane approach to black hole quantum mechanics, Nucl. Phys. B 472 (1996) 591-610, arXiv:hep-th/9602043.

[15] S.D. Mathur, The fuzzball proposal for black holes: An elementary review, Fortschr. Phys. 53 (2005) 793-827, arXiv:hep-th/0502050.

[16] I. Bena, N.P. Warner, Black holes, black rings and their microstates, Lect. Notes Phys. 755 (2008) 1-92, arXiv: hep-th/0701216.

[17] K. Skenderis, M. Taylor, The fuzzball proposal for black holes, Phys. Rep. 467 (2008) 117-171, arXiv:0804.0552 [hep-th].

[18] S.D. Mathur, Fuzzballs and the information paradox: A summary and conjectures, arXiv:0810.4525 [hep-th].

[19] V. Balasubramanian, J. de Boer, S. El-Showk, I. Messamah, Black holes as effective geometries, Class. Quantum Gravity 25 (2008) 214004, arXiv:0811.0263 [hep-th].

[20] B.D. Chowdhury, A. Virmani, Modave lectures on fuzzballs and emission from the D1-D5 system, arXiv:1001.1444 [hep-th].

[21] S.D. Mathur, Black holes and beyond, Ann. Phys. 327 (2012) 2760-2793, arXiv:1205.0776 [hep-th].

[22] O. Lunin, Adding momentum to D1-D5 system, J. High Energy Phys. 0404 (2004) 054, arXiv:hep-th/0404006.

[23] S. Giusto, S.D. Mathur, A. Saxena, Dual geometries for a set of 3-charge microstates, Nucl. Phys. B 701 (2004) 357-379, arXiv:hep-th/0405017.

[24] S. Giusto, S.D. Mathur, A. Saxena, 3-charge geometries and their CFT duals, Nucl. Phys. B 710 (2005) 425-463, arXiv:hep-th/0406103.

[25] I. Bena, N.P. Warner, Bubbling supertubes and foaming black holes, Phys. Rev. D 74 (2006) 066001, arXiv:hep-th/ 0505166.

[26] P. Berglund, E.G. Gimon, T.S. Levi, Supergravity microstates for BPS black holes and black rings, J. High Energy Phys. 0606 (2006) 007, arXiv:hep-th/0505167.

[27] G.W. Gibbons, N.P. Warner, Global structure of five-dimensional BPS fuzzballs, arXiv:1305.0957 [hep-th].

[28] I. Bena, C.-W. Wang, N.P. Warner, The foaming three-charge black hole, Phys. Rev. D 75 (2007) 124026, arXiv: hep-th/0604110.

[29] J. de Boer, S. El-Showk, I. Messamah, D. Van den Bleeken, Quantizing $N=2$ multicenter solutions, J. High Energy Phys. 0905 (2009) 002, arXiv:0807.4556 [hep-th].

[30] J. de Boer, S. El-Showk, I. Messamah, D. Van den Bleeken, A bound on the entropy of supergravity? J. High Energy Phys. 1002 (2010) 062, arXiv:0906.0011 [hep-th].

[31] I. Bena, N. Bobev, S. Giusto, C. Ruef, N.P. Warner, An infinite-dimensional family of black-hole microstate geometries, J. High Energy Phys. 1103 (2011) 022, arXiv:1006.3497 [hep-th].

[32] S. Giusto, R. Russo, D. Turton, New D1-D5-P geometries from string amplitudes, J. High Energy Phys. 1111 (2011) 062, arXiv:1108.6331 [hep-th].

[33] S. Giusto, R. Russo, Perturbative superstrata, Nucl. Phys. B 869 (2013) 164-188, arXiv:1211.1957 [hep-th].

[34] S. Giusto, J.F. Morales, R. Russo, D1D5 microstate geometries from string amplitudes, J. High Energy Phys. 1003 (2010) 130, arXiv:0912.2270 [hep-th].

[35] W. Black, R. Russo, D. Turton, The supergravity fields for a D-brane with a travelling wave from string amplitudes, Phys. Lett. B 694 (2010) 246-251, arXiv:1007.2856 [hep-th].

[36] J. Ford, S. Giusto, A. Saxena, A class of BPS time-dependent 3-charge microstates from spectral flow, Nucl. Phys. B 790 (2008) 258-280, arXiv:hep-th/0612227.

[37] I. Bena, J. de Boer, M. Shigemori, N.P. Warner, Double, double supertube bubble, J. High Energy Phys. 1110 (2011) 116, arXiv:1107.2650 [hep-th].

[38] J.B. Gutowski, D. Martelli, H.S. Reall, All supersymmetric solutions of minimal supergravity in six dimensions, Class. Quantum Gravity 20 (2003) 5049-5078, arXiv:hep-th/0306235.

[39] M. Cariglia, O.A. Mac Conamhna, The general form of supersymmetric solutions of $N=(1,0) \mathrm{U}(1)$ and $\mathrm{SU}(2)$ gauged supergravities in six dimensions, Class. Quantum Gravity 21 (2004) 3171-3196, arXiv:hep-th/0402055.

[40] I. Bena, S. Giusto, M. Shigemori, N.P. Warner, Supersymmetric solutions in six dimensions: A linear structure, J. High Energy Phys. 1203 (2012) 084, arXiv:1110.2781 [hep-th].

[41] I. Kanitscheider, K. Skenderis, M. Taylor, Fuzzballs with internal excitations, J. High Energy Phys. 0706 (2007) 056, arXiv:0704.0690 [hep-th].

[42] S.D. Mathur, A. Saxena, Y.K. Srivastava, Constructing 'hair' for the three charge hole, Nucl. Phys. B 680 (2004) 415-449, arXiv:hep-th/0311092.

[43] L. Martucci, Electrified branes, J. High Energy Phys. 1202 (2012) 097, arXiv:1110.0627 [hep-th].

[44] S. Giusto, R. Russo, Adding new hair to the 3-charge black ring, Class. Quantum Gravity 29 (2012) 085006 , arXiv:1201.2585 [hep-th]. 
[45] O. Vasilakis, Bubbling the newly grown black ring hair, J. High Energy Phys. 1205 (2012) 033, arXiv:1202.1819 [hep-th].

[46] S.D. Mathur, D. Turton, Momentum-carrying waves on D1-D5 microstate geometries, Nucl. Phys. B 862 (2012) 764-780, arXiv:1202.6421 [hep-th].

[47] O. Lunin, S.D. Mathur, D. Turton, Adding momentum to supersymmetric geometries, Nucl. Phys. B 868 (2013) 383-415, arXiv:1208.1770 [hep-th].

[48] B.E. Niehoff, O. Vasilakis, N.P. Warner, Multi-superthreads and supersheets, arXiv:1203.1348 [hep-th].

[49] O. Vasilakis, Corrugated multi-supersheets, J. High Energy Phys. 1307 (2013) 008, arXiv:1302.1241 [hep-th].

[50] S.D. Mathur, D. Turton, Microstates at the boundary of AdS, J. High Energy Phys. 1205 (2012) 014, arXiv:1112. 6413 [hep-th].

[51] A. Dabholkar, J.P. Gauntlett, J.A. Harvey, D. Waldram, Strings as solitons \& black holes as strings, Nucl. Phys. B 474 (1996) 85-121, arXiv:hep-th/9511053.

[52] C.G. Callan, J.M. Maldacena, A.W. Peet, Extremal black holes as fundamental strings, Nucl. Phys. B 475 (1996) 645-678, arXiv:hep-th/9510134.

[53] O. Lunin, S.D. Mathur, Metric of the multiply wound rotating string, Nucl. Phys. B 610 (2001) 49-76, arXiv: hep-th/0105136.

[54] O. Lunin, S.D. Mathur, AdS/CFT duality and the black hole information paradox, Nucl. Phys. B 623 (2002) 342-394, arXiv:hep-th/0109154.

[55] J.M. Maldacena, Black holes in string theory, arXiv:hep-th/9607235.

[56] J.R. David, G. Mandal, S.R. Wadia, Microscopic formulation of black holes in string theory, Phys. Rep. 369 (2002) 549-686, arXiv:hep-th/0203048.

[57] V. Balasubramanian, J. de Boer, E. Keski-Vakkuri, S.F. Ross, Supersymmetric conical defects: Towards a string theoretic description of black hole formation, Phys. Rev. D 64 (2001) 064011, arXiv:hep-th/0011217.

[58] O. Lunin, J.M. Maldacena, L. Maoz, Gravity solutions for the D1-D5 system with angular momentum, arXiv: hep-th/0212210.

[59] B.E. Niehoff, N.P. Warner, Doubly-fluctuating BPS solutions in six dimensions, arXiv:1303.5449 [hep-th].

[60] E. Bergshoeff, R. Kallosh, T. Ortin, D. Roest, A. Van Proeyen, New formulations of $D=10$ supersymmetry and D8-O8 domain walls, Class. Quantum Gravity 18 (2001) 3359-3382, arXiv:hep-th/0103233.

[61] D. Lust, F. Marchesano, L. Martucci, D. Tsimpis, Generalized non-supersymmetric flux vacua, J. High Energy Phys. 0811 (2008) 021, arXiv:0807.4540 [hep-th]. 